
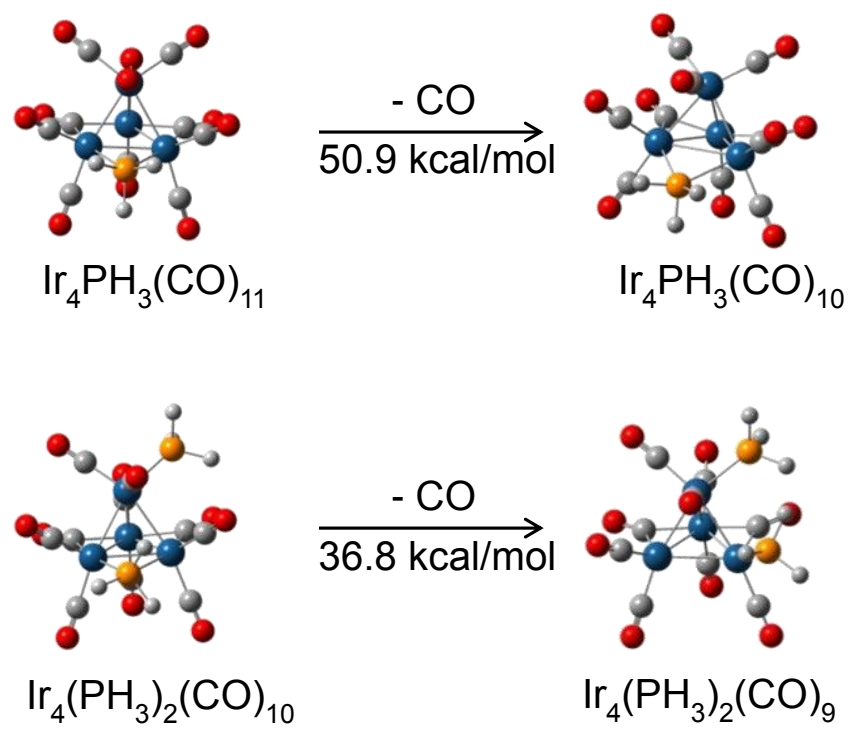

The calculated ligand dissociation energies of $\operatorname{IR}_{\mathrm{X}}\left(\mathrm{PH}_{3}\right)_{\mathrm{Y}}(\mathrm{CO})_{\mathrm{Z}}(\mathrm{X}=1,2,4)$ provide insights into the bonding and structures of these complexes relevant to single site catalysis. 


\title{
Structures, Relative Energies, and Ligand Dissociation Energies of Iridium Carbonyl Phosphine Clusters
}

\author{
Shengjie Zhang ${ }^{a}$, Alexander Katz ${ }^{b}$, Bruce C. Gates ${ }^{c}$, David A. Dixon ${ }^{a, *}$ \\ ${ }^{a}$ Department of Chemistry, The University of Alabama, Tuscaloosa, Alabama 35487, United States. \\ ${ }^{\mathrm{b}}$ Department of Chemical and Biomolecular Engineering, University of California at Berkeley, Berkeley, \\ California, 94720, United States. \\ ${ }^{c}$ Department of Chemical Engineering and Materials Science, University of California at Davis, Davis, \\ California 95616, United States.
}

\begin{abstract}
There is significant interest in the catalytic properties of substituted iridium carbonyl clusters but little thermodynamic information available characterizing them. The low-energy isomers of $\operatorname{Ir}_{\mathrm{x}}\left(\mathrm{PH}_{3}\right)_{\mathrm{y}}(\mathrm{CO})_{\mathrm{z}}(\mathrm{x}=1,2$, 4) were investigated with density functional theory and correlated molecular orbital theory at the coupled cluster CCSD(T) level. The relative energies and ligand dissociation energies were calculated. Differences in relative energies are consequences of both electronic and steric effects of the phosphines and carbonyls. The calculations predict three fundamental structural types for $\operatorname{Ir}_{2}\left(\mathrm{PH}_{3}\right)_{\mathrm{y}}(\mathrm{CO})_{\mathrm{z}}: \mathrm{C}_{2 \mathrm{v}}, \mathrm{C}_{2}$, and $\mathrm{D}_{3 \mathrm{~d}}$. Ten exchange-correlation functionals were used for the ligand dissociation energy calculations in addition to $\operatorname{CCSD}(\mathrm{T})$ for the smaller clusters. The $\omega \mathrm{B} 97 \mathrm{X}-\mathrm{D}$ functional gave the most consistent ligand dissociation energies as compared with the CCSD(T) benchmark calculations, and, so it was used to predict the dissociation energies for larger clusters when $\operatorname{CCSD}(\mathrm{T})$ calculations were infeasible. The dissociation energies characteristic of $\operatorname{Ir}_{4}\left(\mathrm{PH}_{3}\right)_{\mathrm{y}}(\mathrm{CO})_{\mathrm{z}}$ were in the range of $\sim 30$ to $\sim 60 \mathrm{kcal} / \mathrm{mol}$. Dissociation of a bridging ligand often involved a hydrogen atom transfer from a phosphine to a coordinatively unsaturated iridium atom and a phosphine converting from a bridging site to an equatorial site. The products of such reactions are predicted to have lower relative energies than other isomers. Phosphines act as $\sigma$-electron donors, and there is a trend of an increase in carbonyl ligand dissociation energies as more phosphines are substituted in the small clusters.
\end{abstract}

Keywords: iridium carbonyl phosphine complexes; electronic structure calculations; ligand dissociation energies; coupled cluster $(\mathrm{CCSD}(\mathrm{T}))$ theory; density functional theory; catalysis

\section{Introduction}

\footnotetext{
* Corresponding author.

E-mail address: dadixon@ua.edu (D. A. Dixon)
} 
Iridium clusters catalyze a wide range of reactions, including oxidation, hydrogenation, $\mathrm{C}-\mathrm{H}$ bond activation, cycloaddition, cycloisomerization, [1,2] and ring-opening.[3] Iridium complexes play important roles in oxidation (e.g., of alcohols, $[4,5,6]$ phenols, $[7,8,9]$ and amines[10,11]), hydrogenation,[12,13] C-H activation, $[14,15,16]$ cycloaddition (e.g., $[2+2+2],[17,18,19][2+2+1],[20,21]$ and [4 + 2] [22,23]), cycloisomerization,[1,2] and ring-opening reactions.[24] The richness of this chemistry explains the broad interest in the properties of the family of iridium complexes and clusters.[25] Ozin and coworkers [26,27,28] reported the synthesis of $\operatorname{Ir}(\mathrm{CO})_{\mathrm{n}}(\mathrm{n}=1-4)$ and $\mathrm{Ir}_{2}(\mathrm{CO})_{8}$ clusters in a matrix. $\mathrm{Ir}_{2}(\mathrm{CO})_{8}$ was reported to transform into the tetra-iridium carbonyl cluster $\operatorname{Ir}_{4}(\mathrm{CO})_{12}$ at $\sim 200$ K.[26,27] The tetra-iridium cluster framework is one of the simplest structures presenting neighbouring metal centers, and numerous tetra-iridium clusters are known. The chemistry of tetra-iridium carbonyl clusters is especially extensive, as the metal frame is relatively stable, so that many compounds in this class have been investigated, including those that are catalysts both in solution and on supports.[29,30,31,32] The parent tetra-iridium cluster $\operatorname{Ir}_{4}(\mathrm{CO})_{12}$ has two structures which are minima on the potential energy surface, one $T_{d}$ structure with all 12 carbonyls being terminal, and one $C_{3 v}$ structure with nine terminal carbonyls and three bridging carbonyls on the basal plane (Figure 1). Different from $\mathrm{Co}_{4}(\mathrm{CO})_{12}$ and $\mathrm{Rh}_{4}(\mathrm{CO})_{12}$, which adopt $\mathrm{C}_{3 \mathrm{v}}$ symmetry $[33,34]$ both in the gas phase and in solution, $\operatorname{Ir}_{4}(\mathrm{CO})_{12}$ adopts the $\mathrm{T}_{\mathrm{d}}$ structure.[35,36,37] A single-crystal X-ray diffraction investigation yielded $r(\mathrm{Ir}-\mathrm{Ir})=2.693 \AA$ and $r(\mathrm{Ir}-\mathrm{C})=$ $1.87 \AA$ A.[36] An extended Hückel investigation of tetrahedral $\mathrm{M}_{4} \mathrm{CO}_{11} \mathrm{~L}$ clusters for $\mathrm{M}$ $=\mathrm{Co}, \mathrm{Rh}$, and Ir was combined with an analysis of reported crystal structure data to provide insights into the cluster properties, including the role of the ligand $\mathrm{L}$ on determining if bridging CO ligands are present.[38]

When a carbonyl is replaced by a phosphine ligand L, the cluster usually [39] shows a preference for the $\mathrm{C}_{3 \mathrm{v}}$-type structure, and can adopt the structure of either isomer $\mathbf{b}$ or c (Figure 1) if the phosphine ligand L is small; isomer $\mathbf{b}$ becomes preferred as the bulk of L increases.[40] Electronic effects may also be significant in this chemistry, reflecting the electron withdrawing or donating tendencies of the groups bonded to the phosphorus.[38] When $\mathrm{L}$ is a phosphine or phosphite, its bulk also strongly influences the degree of ligand substitution in the parent cluster $\operatorname{Ir}_{4}(\mathrm{CO})_{12}$-that is, determining whether $\operatorname{Ir}_{4}(\mathrm{CO})_{8} \mathrm{~L}_{4}$ or $\operatorname{Ir}_{4}(\mathrm{CO})_{9} \mathrm{~L}_{3}$ is the final product[41] -but synthesis of $\operatorname{Ir}_{4}(\mathrm{CO})_{10} \mathrm{~L}_{2}$ or $\mathrm{Ir}_{4}(\mathrm{CO})_{11} \mathrm{~L}$ directly from the parent through direct substitution is difficult.[41] Okrut et al. [42] demonstrated that as the number of strong $\sigma$-donor phosphine ligands increases, the thermomechanical stability against aggregation increases, whereas the thermochemical stability against decarbonylation varies inversely.

We were particularly motivated to investigate tetrairidium clusters with combinations of carbonyl and phosphine ligands, having recently demonstrated an on-off switch for the bonding and catalytic conversion of ethylene at single iridium sites on the tetrairidium frame, as triggered by a sheath of organic ligands bonded at neighboring iridium sites that control the electronic properties of the active site.[43] Ethylene binding and activation in a supported tetrairidium cluster catalyst were shown to be controlled by selective nanoenvironments at the metal surface, which are formed by three bulky calixarene-phosphine ligands surrounding and bonding to the cluster at sites neighbouring the site where reactivity is switched off and on. These 
results point to the importance of understanding the bonding and energetics of $\mathrm{CO} /$ phosphine complexes of tetrairidium. However, the energetics of iridium carbonyl phosphine complexes are currently not available, either from experiment or theory.

We now report the structures and bond dissociation energies $\operatorname{of} \operatorname{Ir}_{\mathrm{x}}\left(\mathrm{PH}_{3}\right)_{\mathrm{y}}(\mathrm{CO})_{\mathrm{z}}$ for values of $\mathrm{x}$ up to 4 determined by using density functional theory (DFT) $[44,45,46]$ and coupled cluster theory $(\operatorname{CCSD}(\mathrm{T}))[47,48,49,50]$ to provide insights into the bonding of the simplest phosphine in such complexes. We compare the results determined with various DFT exchange-correlation functionals to provide a guide for their use with larger substituted phosphines. These results lead to a better understanding of carbonyl and phosphine chemisorption on iridium clusters and provide an improved basis for ultimately predicting their electronic structures and catalytic properties. In addition, the calculated structural and energetic information can be used as an aid in identifying various species that could be synthesized in matrix isolation experiments.

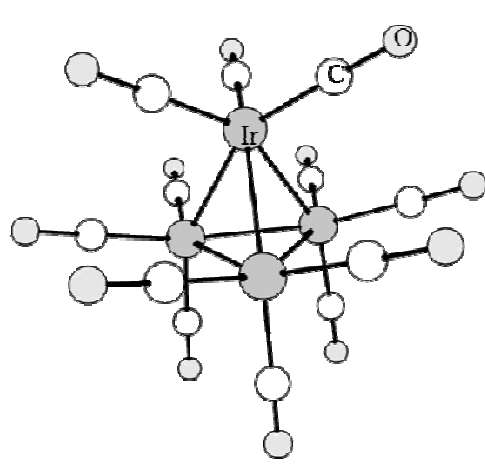

a

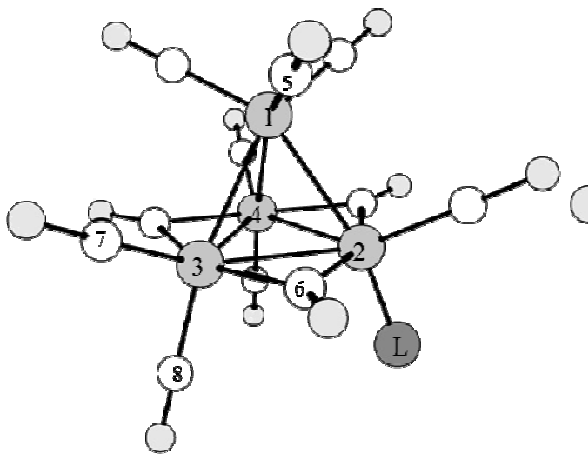

b

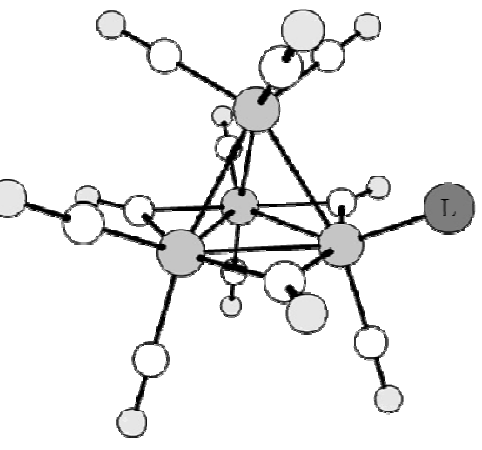

C

Figure 1: Isomers of $\operatorname{Ir}_{4}(\mathrm{CO})_{12}$ and $\mathrm{Ir}_{4}(\mathrm{CO})_{11} \mathrm{~L}$. In $\mathrm{T}_{\mathrm{d}}$ structure a, all carbonyls are terminal. In $\mathrm{C}_{3 \mathrm{v}}$ structure b, $\operatorname{Ir}(1)$ is apical iridium, and $\operatorname{Ir}(2), \operatorname{Ir}(3)$, and $\operatorname{Ir}(4)$ are basal iridiums that build up the basal plane. $\mathrm{C}(5), \mathrm{C}(6), \mathrm{C}(7)$, and $\mathrm{C}(8)$ are apical, bridging, equatorial, and axial carbon, respectively. $\mathrm{L}$ is the ligand that substitutes for axial carbonyl in $\mathbf{b}$ and for equatorial carbonyl in $\mathbf{c}$.

\section{Computational Methods}

Previously, geometry parameters of $\operatorname{Ir}_{4}(\mathrm{CO})_{12}$ with $\mathrm{T}_{\mathrm{d}}$ symmetry have been obtained from DFT with the SVWN5 exchange-correlation functional, which provides excellent geometry results that are very close to the experimental results, but gives poor relative energies that predict a lower-energy $\mathrm{C}_{3 \mathrm{v}}$ structure.[51] The CAM-B3LYP functional performs best in calculations of carbonyl dissociation energies for a number of $\operatorname{Ir}_{\mathrm{x}}(\mathrm{CO})_{\mathrm{y}}$ complexes, and the $\omega \mathrm{B} 97 \mathrm{X}-\mathrm{D}$ functional gives the best predictions of total dissociation energies.[51] In most nucleation reactions, DFT calculations give higher energies than CCSD(T) results, whereas the reverse pertains to the prediction based on the MP2 method. Thus, the average of the MP2 and DFT values were considered to be suitable for predicting the carbonyl ligand dissociation energies.[51]

Following our previous work, the geometries of the $\operatorname{Ir}_{\mathrm{x}}\left(\mathrm{PH}_{3}\right)_{\mathrm{y}}(\mathrm{CO})_{\mathrm{z}}$ clusters were optimized by using DFT with the local density approximation functional SVWN5 
(Slater exchange plus the VWN version 5 fit of the electron gas for the correlation potential).[52,53] Second-derivative frequency calculations were performed on every optimized geometry to confirm that the specific structure is a minimum.

Both the DFT and single-point $\operatorname{CCSD}(\mathrm{T})$ calculations were performed with the augmented correlation consistent double- $\zeta$ (aug-cc-pVDZ) basis set[54,55] for light elements and a relativistic pseudopotential with the aug-cc-pVDZ-pp basis set for iridium.[56] This combination is denoted as the ' $\mathrm{aD}$ ' basis set. The PP on Ir includes the following 60 electrons in the PP: $1 s^{2} 2 s^{2} 2 p^{6} 3 s^{2} 3 p^{6} 3 d^{10} 4 s^{2} 4 p^{6} 4 d^{10} 4 f^{14}$. The active electrons in the self-consistent field calculations for the Ir atom are the $5 s^{2} 5 p^{6} 6 s^{2} 5 d^{7}$. In the valence $\operatorname{CCSD}(\mathrm{T})$ and MP2 calculations, the $5 \mathrm{~s}^{2} 5 \mathrm{p}^{6}$ are in the core together with the $1 \mathrm{~s}$ on $\mathrm{C}$ and $\mathrm{O}$ and the $1 \mathrm{~s}^{2} 2 \mathrm{~s}^{2} 2 \mathrm{p}^{6}$ on the $\mathrm{P}$.

Single-point energies of the clusters were calculated by using $\operatorname{CCSD}(\mathrm{T})$ and DFT with the following exchange-correlation functionals: B3LYP,[57,58] B97-D,[59] B98,[60,61] BP86,[62] CAM-B3LYP,[63] M06,[64] PW91,[65,66,67] PBE,[68,69] $\omega \mathrm{B} 97 \mathrm{X},[70]$ and $\omega \mathrm{B} 97 \mathrm{X}-\mathrm{D} .[71] \mathrm{BP} 86, \mathrm{PBE}$, and PW91 are pure generalized gradient approximation (GGA) functionals; B3LYP, B98, and M06 are hybrid functionals; B97-D is a GGA functional with dispersion corrections; CAM-B3LYP and $\omega$ B97X are hybrid functionals with long-range corrections; and $\omega \mathrm{B} 97 \mathrm{X}-\mathrm{D}$ is a hybrid functional with long-range corrections and dispersion corrections. The CCSD(T) energies of the open-shell molecules $\operatorname{Ir}\left(\mathrm{PH}_{3}\right)_{\mathrm{y}}(\mathrm{CO})_{\mathrm{z}}$ were calculated by using the R/UCCSD(T) approach.[72,73,74] The open shell MP2 calculations were done at the ROMP2 level.[75]

Ligand dissociation energies (LDEs) were calculated from equation (1).

$$
\mathrm{LDE}=E(\text { product })+E(\text { ligand })-E \text { (parent cluster })
$$

All LDEs are electronic energies with zero point energy correction included at $0 \mathrm{~K}$ giving $\Delta H(0 \mathrm{~K})$.

The DFT calculations were performed with the Gaussian09 suite of programs[76] and the MP2 and CCSD(T) calculations with the MOLPRO2010 suite of programs.[77]

\section{Results and Discussion}

\section{1 $\operatorname{Ir}\left(\mathrm{PH}_{3}\right)_{\mathrm{y}}(\mathrm{CO})_{\mathrm{z}}$ structures, relative energies, and ligand dissociation energies.}

First consider the isomers of $\operatorname{Ir}\left(\mathrm{PH}_{3}\right)_{y}(\mathrm{CO})_{\mathrm{z}}$ shown in Figure 2. The optimized bond distances are given in the Supporting Information (SI), and the relative energies are listed in Table 1. Quartet states of $\operatorname{Ir}\left(\mathrm{PH}_{3}\right)_{y}(\mathrm{CO})_{z}$ are of higher energy than doublet states for each structure, and so only the doublet states are compared in this work. The LDEs of the $\operatorname{Ir}\left(\mathrm{PH}_{3}\right)_{\mathrm{y}}(\mathrm{CO})_{\mathrm{z}}$ complexes are listed in Table 2.

The $\operatorname{Ir}(\mathrm{CO})_{2}, \quad \operatorname{IrPH}{ }_{3} \mathrm{CO}$, and $\operatorname{Ir}\left(\mathrm{PH}_{3}\right)_{2}$ molecules have two minimum energy structures, one bent and one almost linear. The bent structures are lower in energy by $9.9,6.8$, and $5.9 \mathrm{kcal} / \mathrm{mol}$, respectively. $\operatorname{Ir}(\mathrm{CO})_{3}$ has an almost planar geometry with one equatorial $(e q=$ equatorial $) \mathrm{CO}$ and two axial $(a x=$ axial $) \mathrm{CO}$ ligands. As one $\mathrm{PH}_{3}$ is substituted for a $\mathrm{CO}$, two $\mathrm{IrPH}_{3}(\mathrm{CO})_{2}$ isomers are formed, one with an axial $\mathrm{PH}_{3}$ $(a x, 1 b)$, which has a lower energy by $2.2 \mathrm{kcal} / \mathrm{mol}(\operatorname{CCSD}(\mathrm{T}))$, and one with an equatorial $\mathrm{PH}_{3}(e q, \mathbf{1 c})$. In $\mathbf{1 b}$, the PIrCC plane is maintained, whereas the two carbonyls bend slightly out of the plane $\left(\angle \mathrm{C}\right.$-Ir-P-C $\left.=153^{\circ}\right)$ in $\mathbf{1 c}$ as a consequence of 


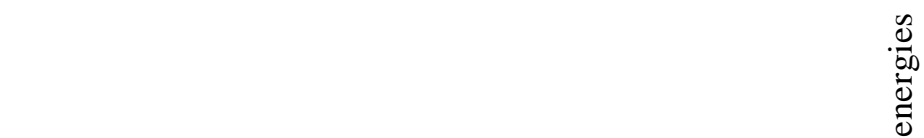

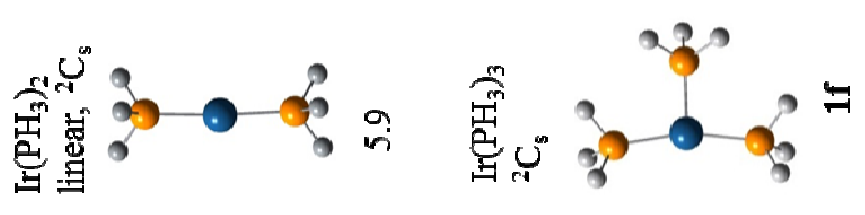

$$
\begin{aligned}
& \text { 密垔望 }
\end{aligned}
$$

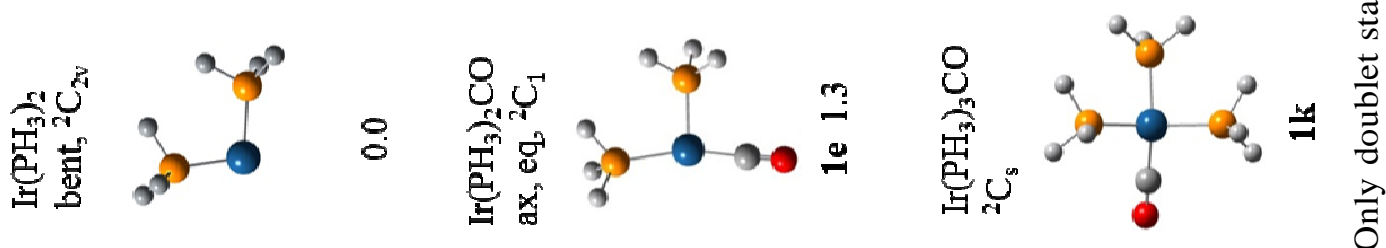

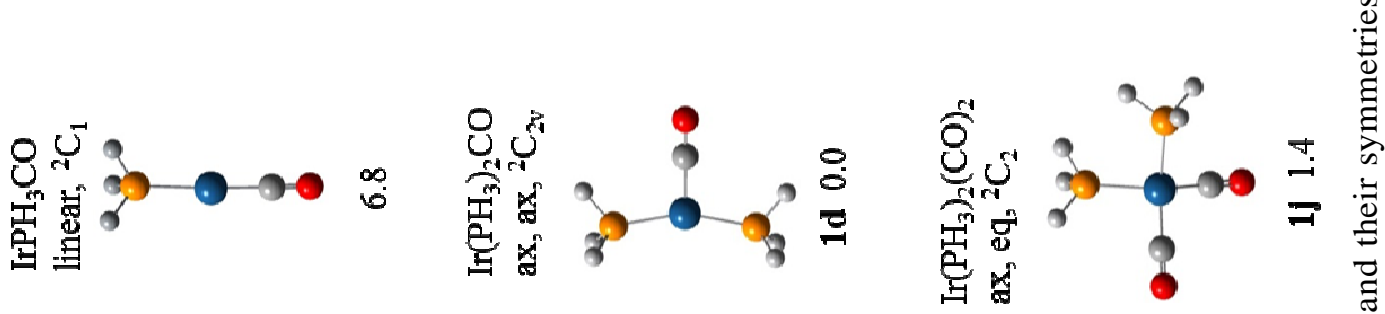

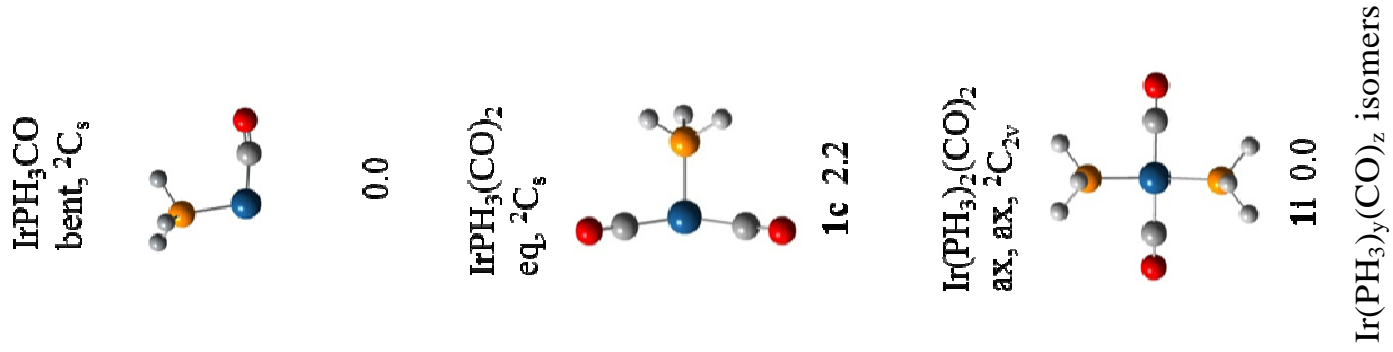

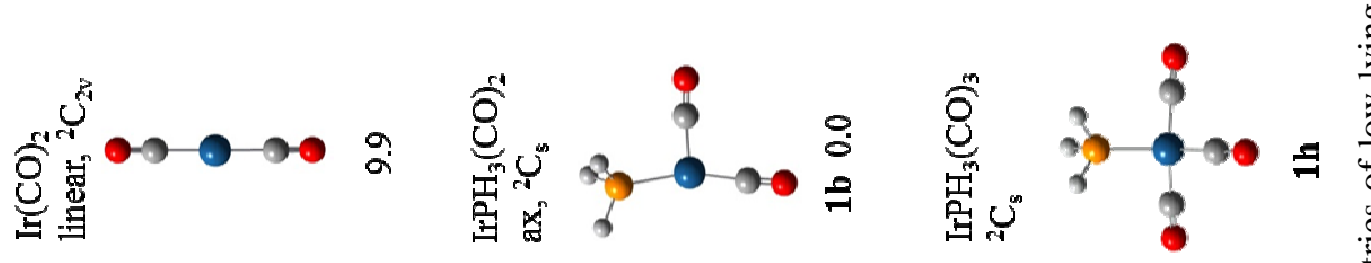

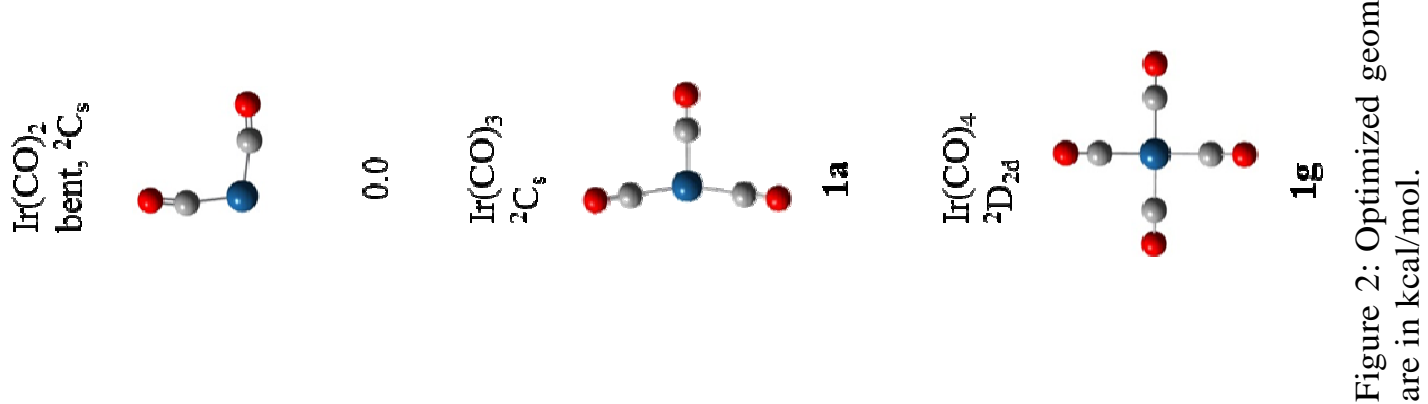




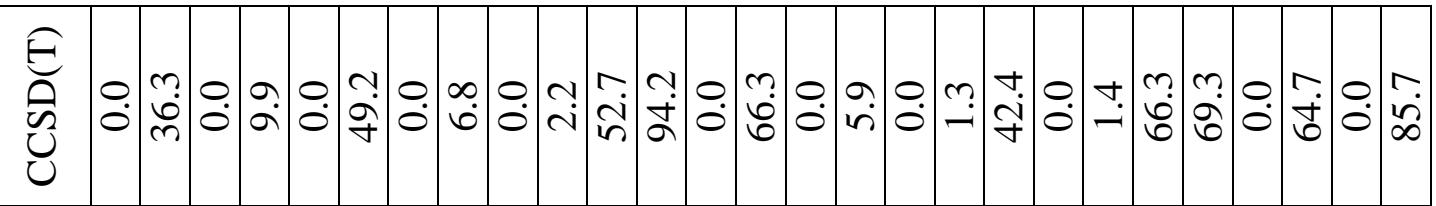

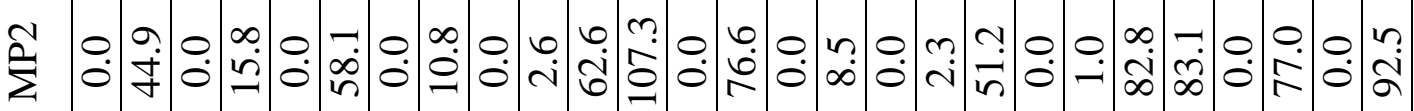

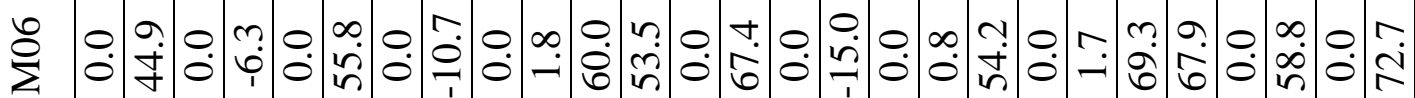

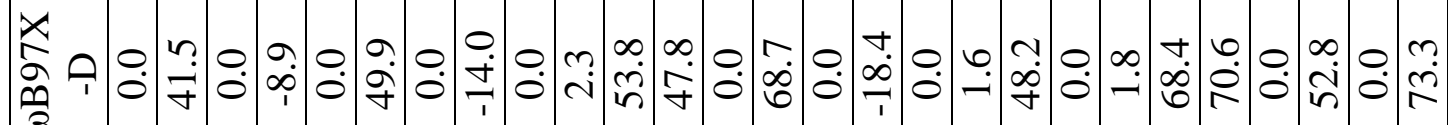
3

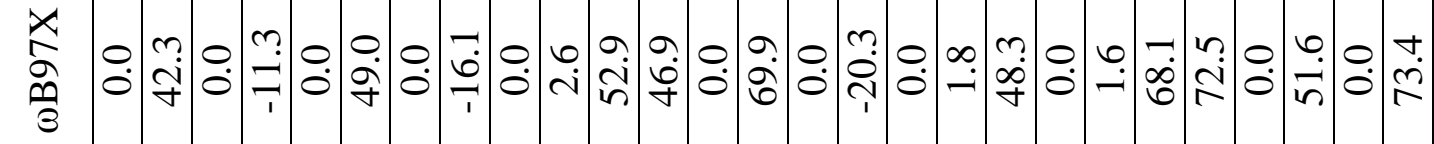

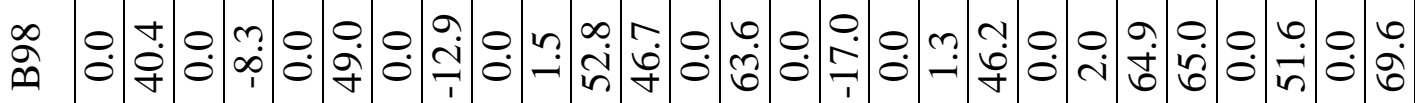

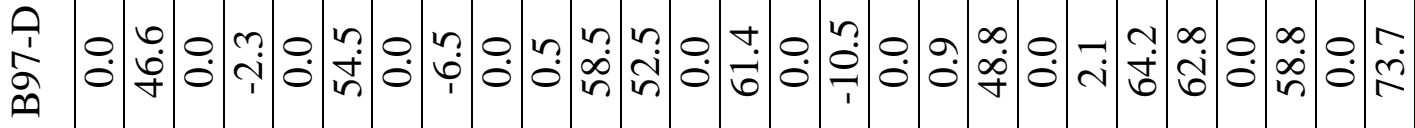

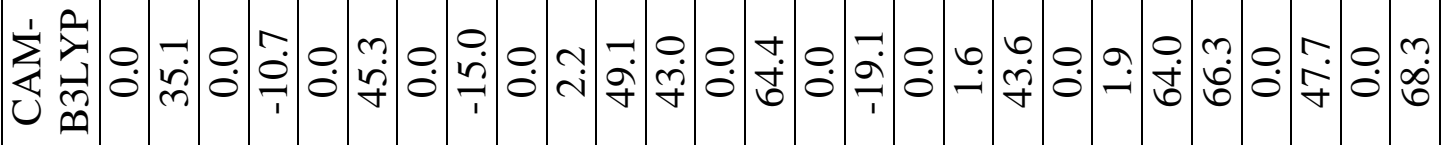

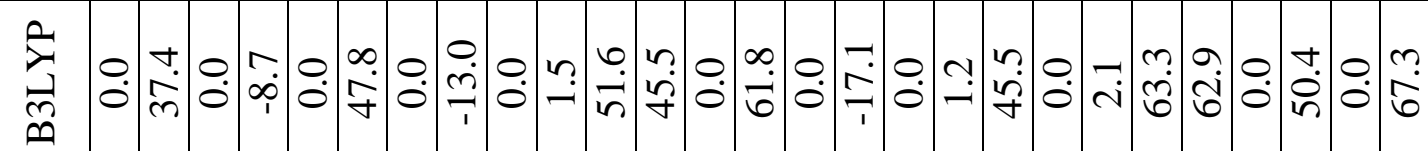

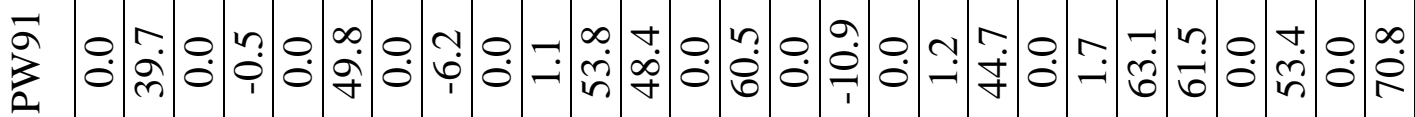

垤

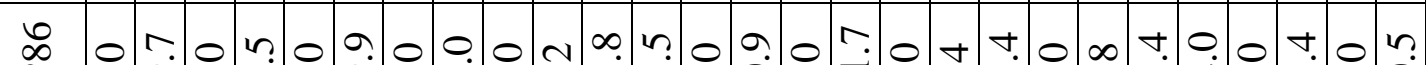

乔

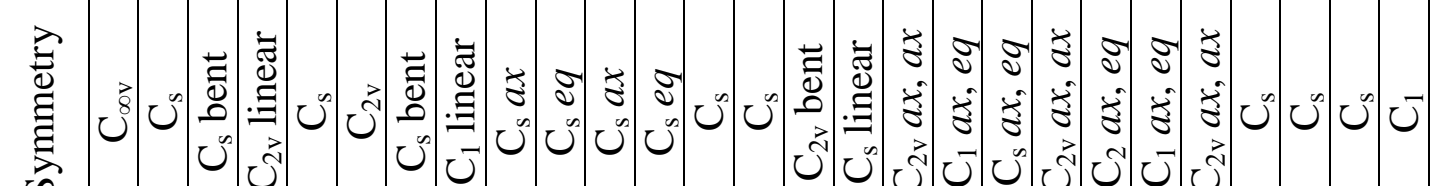

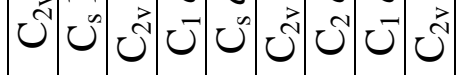

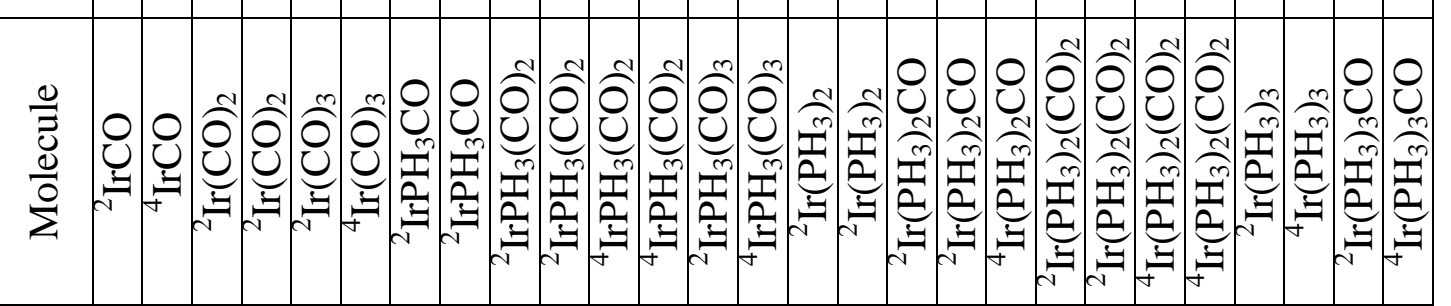




\begin{tabular}{|c|c|c|c|c|c|c|c|c|c|c|c|c|c|c|}
\hline$\underset{\tilde{E}}{\stackrel{\Xi}{己}}$ & $\stackrel{m}{\ni}$ & $\frac{N}{n}$ & $\vec{\infty}$ & 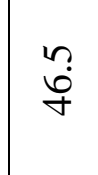 & $\begin{array}{l}m \\
m \\
n\end{array}$ & $\begin{array}{l}\infty \\
\stackrel{\infty}{f}\end{array}$ & $\stackrel{m}{\forall}$ & $\stackrel{\nabla}{+}$ & ஓें & $\vec{n}$ & $\stackrel{\vartheta}{\dot{\vartheta}}$ & $\stackrel{n}{n}$ & $\begin{array}{l}\infty \\
\stackrel{\infty}{ }\end{array}$ & $\begin{array}{l}0 \\
\dot{\theta} \\
\dot{\gamma}\end{array}$ \\
\hline$\stackrel{\Sigma}{\Sigma}$ & $\underset{\infty}{\stackrel{\infty}{+}}$ & $\begin{array}{l}\circ \\
\dot{0}\end{array}$ & $\stackrel{m}{y}$ & $\begin{array}{l}0 \\
\dot{n} \\
i n\end{array}$ & $\underset{8}{\forall}$ & $\begin{array}{l}0 \\
\infty \\
\infty\end{array}$ & $\begin{array}{l}0 \\
\hat{n}\end{array}$ & $\frac{\mathfrak{r}}{\gamma}$ & $\stackrel{\nabla}{\sim}$ & $\stackrel{q}{\dot{y}}$ & in & $\tilde{\sigma}$ & $\tilde{q}$ & $\begin{array}{l}0 \\
\infty \\
i n\end{array}$ \\
\hline$\stackrel{\wp}{\stackrel{2}{2}}$ & $\stackrel{n}{\sim}$ & ণ্ & $\begin{array}{l}0 \\
\dot{\vec{m}}\end{array}$ & $\frac{n}{\forall}$ & 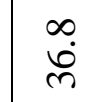 & $\stackrel{n}{n}$ & $\stackrel{r}{\forall}$ & $\begin{array}{l}0 \\
\text { mे }\end{array}$ & $\stackrel{m}{m}$ & $\stackrel{r}{m}$ & ๙̃ & 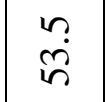 & $\dot{n}$ & $\dot{\overbrace{}}$ \\
\hline $\begin{array}{l}\underset{\alpha}{\alpha} \\
\frac{\hat{\sigma}}{3}\end{array}$ & $\stackrel{n}{\nexists}$ & $\begin{array}{l}\dot{b} \\
\ddot{n}\end{array}$ & $\begin{array}{l}0 \\
\infty \\
\infty \\
m\end{array}$ & $\ddot{\circ}$ & $\vec{b}$ & $\stackrel{\Delta}{\stackrel{\Delta}{N}}$ & $\frac{a}{\sigma}$ & $\begin{array}{l}0 \\
\infty \\
\infty \\
m\end{array}$ & ๙ุ? & $\underset{n}{0}$ & 옴 & $\begin{array}{l}\stackrel{0}{0} \\
\stackrel{n}{n}\end{array}$ & $\begin{array}{l}\infty \\
\stackrel{\infty}{ }\end{array}$ & $\stackrel{\nabla}{\sim}$ \\
\hline $\begin{array}{l}\frac{x}{\hat{a}} \\
\frac{1}{3}\end{array}$ & 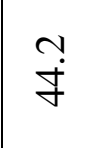 & $\begin{array}{l}0 \\
\dot{m}\end{array}$ & ণे. & $\begin{array}{l}0 \\
\stackrel{q}{q}\end{array}$ & $\stackrel{n}{m}$ & $\stackrel{n}{n}$ & वें & ๙े & n̊? & $\underset{m}{\stackrel{\nabla}{m}}$ & $\frac{n}{\pi}$ & $\begin{array}{l}0 \\
i n \\
i n\end{array}$ & $\stackrel{\circ}{\circ}$ & $\stackrel{b}{9}$ \\
\hline$\stackrel{\infty}{\emptyset}$ & $\underset{f}{\exists}$ & $\ddot{n}$ & $\ddot{n}$ & $\stackrel{ナ}{\stackrel{\sigma}{\sigma}}$ & $\begin{array}{l}0 \\
\stackrel{0}{0} \\
\text { లn }\end{array}$ & $\stackrel{r}{\sim}$ & $\stackrel{\circ}{\stackrel{\infty}{+}}$ & $\stackrel{m}{m}$ & ڤें & mें & $\frac{\sim}{\sim}$ & $\begin{array}{l}\dot{n} \\
\ddot{n}\end{array}$ & $\begin{array}{l}0 \\
\stackrel{0}{0}\end{array}$ & $\stackrel{0}{9}$ \\
\hline$\hat{\bar{\theta}}$ & $\ddot{\vartheta}$ & $\stackrel{\infty}{\dot{q}}$ & $\begin{array}{l}\infty \\
\dot{m}\end{array}$ & 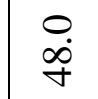 & $\stackrel{n}{7}$ & @़े & $\frac{n}{f}$ & $\stackrel{\vec{m}}{m}$ & $\ddot{f}$ & $\begin{array}{l}o \\
\text { ஸे }\end{array}$ & $\vec{d}$ & $\ddot{n}$ & $\begin{array}{l}0 \\
\text { n. }\end{array}$ & $\stackrel{n}{\sim}$ \\
\hline 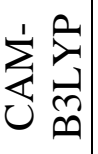 & $\begin{array}{l}0 \\
\dot{f}\end{array}$ & $\stackrel{m}{m}$ & 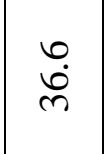 & $\stackrel{\circ}{\stackrel{+}{q}}$ & $\ddot{m}$ & $\begin{array}{l}\infty \\
\dot{\sim}\end{array}$ & $\begin{array}{l}\infty \\
\dot{\varphi}\end{array}$ & mo & $\underset{n}{\infty}$ & $\begin{array}{l}0 \\
\dot{+}\end{array}$ & $\begin{array}{l}0 \\
\stackrel{0}{ }\end{array}$ & $\stackrel{n}{+}$ & बें & $\stackrel{\infty}{\perp}$ \\
\hline 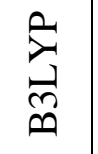 & 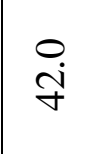 & $\stackrel{\vec{r}}{\dot{m}}$ & ì & $\stackrel{r}{r}$ & $\begin{array}{l}0 \\
\dot{\sim}\end{array}$ & $\stackrel{n}{\sim}$ & $\stackrel{n}{f}$ & $\stackrel{m}{m}$ & बें & $\stackrel{\leftrightarrows}{m}$ & $\ddot{\infty}$ & $\begin{array}{l}\infty \\
i \\
i \\
n\end{array}$ & $\stackrel{\nabla}{\dot{m}}$ & ?ֶ? \\
\hline $\bar{a}^{\bar{a}}$ & $\stackrel{\infty}{a}$ & ๙̣̊ & $\underset{\infty}{\infty}$ & $\ddot{n}$ & $\tilde{q}$ & $\begin{array}{l}0 \\
\text { ம}\end{array}$ & $\begin{array}{l}0 \\
\dot{\sim} \\
n\end{array}$ & $\vec{\infty}$ & กั & बें & $\stackrel{\infty}{\infty}$ & $\frac{9}{6}$ & 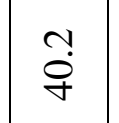 & ๙ุ \\
\hline$\frac{\Gamma}{n}$ & $\stackrel{q}{q}$ & $\stackrel{\Upsilon}{q}$ & $\frac{0}{n}$ & 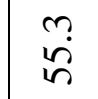 & $\stackrel{\forall}{\dot{q}}$ & $\begin{array}{l}\infty \\
\stackrel{n}{n}\end{array}$ & $\vec{n}$ & $\frac{0}{n}$ & $\overrightarrow{i n}$ & 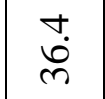 & $\begin{array}{l}\dot{0} \\
\dot{m}\end{array}$ & $\frac{\dot{\sigma}}{\sigma}$ & $\hat{\sigma}$ & $\overrightarrow{\mathrm{d}}$ \\
\hline $\begin{array}{l}0 \\
\infty \\
\infty\end{array}$ & $\underset{\stackrel{\infty}{+}}{\stackrel{\infty}{+}}$ & $\begin{array}{l}n \\
\text { no }\end{array}$ & $\vec{n}$ & $\begin{array}{l}\infty \\
\dot{n} \\
\dot{n}\end{array}$ & $\begin{array}{l}\infty \\
\dot{0} \\
\dot{f}\end{array}$ & $\vec{m}$ & ñ & ণై & $\stackrel{\nabla}{\dot{\sigma}}$ & $\begin{array}{l}\infty \\
\dot{m}\end{array}$ & $\underset{\sim}{\infty}$ & $\begin{array}{l}\infty \\
\stackrel{\circ}{n}\end{array}$ & $\frac{a}{m}$ & ণֶ \\
\hline 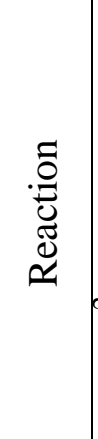 & 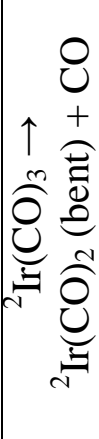 & 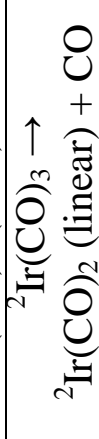 & 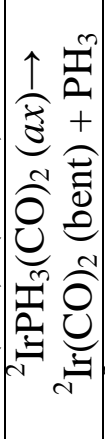 & 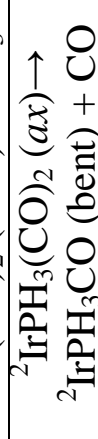 & 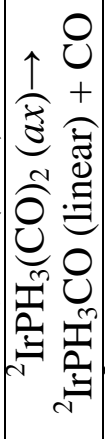 & 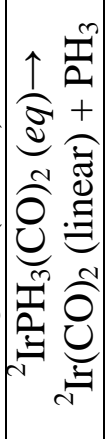 & 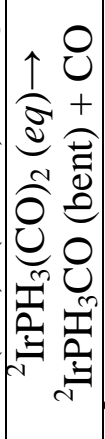 & 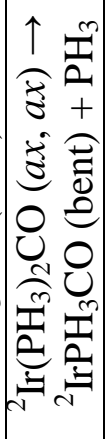 & 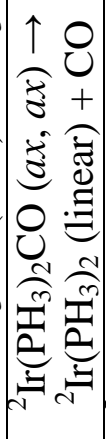 & 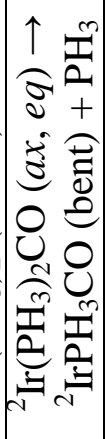 & 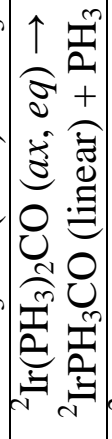 & 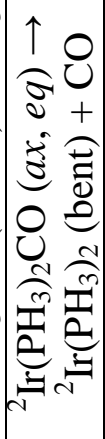 & 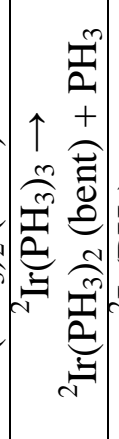 & 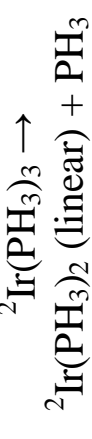 \\
\hline
\end{tabular}




\begin{tabular}{|c|c|c|c|c|c|c|c|c|c|c|c|c|}
\hline 己ั & $\begin{array}{l}n \\
\stackrel{\infty}{N}\end{array}$ & 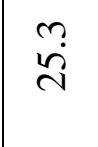 & $\begin{array}{l}n \\
\infty \\
\sim\end{array}$ & @ே. & ते & $\frac{\infty}{m}$ & ָั & $\frac{0}{n}$ & $\begin{array}{l}0 \\
\dot{n} \\
\dot{n}\end{array}$ & $\vec{\vartheta}$ & $\stackrel{\infty}{\sim}$ & 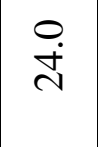 \\
\hline$\stackrel{\Sigma}{\Sigma}$ & $\begin{array}{l}\infty \\
\infty \\
\infty\end{array}$ & n? & ஜे & $\stackrel{\circ}{\stackrel{ }{f}}$ & $\stackrel{+}{\dot{m}}$ & $\stackrel{\infty}{\stackrel{\sim}{f}}$ & $\dot{m}$ & 尹̈ & $\ddot{\infty}$ & $\stackrel{n}{n}$ & $\stackrel{\sim}{m}$ & $\begin{array}{l}\infty \\
\dot{0} \\
\ddot{n}\end{array}$ \\
\hline$\stackrel{8}{\stackrel{2}{z}}$ & $\begin{array}{l}\infty \\
\stackrel{\lambda}{~}\end{array}$ & $\stackrel{ָ}{ָ}$ & 요 & $\stackrel{\infty}{\dot{m}}$ & $\stackrel{n}{\stackrel{n}{~}}$ & $\begin{array}{l}\infty \\
\dot{m}\end{array}$ & $\begin{array}{l}0 \\
\infty \\
\infty\end{array}$ & ते & $\frac{\pi}{n}$ & $\stackrel{n}{=}$ & $\ddot{\infty}$ & $\underline{a}$ \\
\hline $\begin{array}{l}x \\
\frac{x}{\sigma} \\
\frac{\hat{n}}{3}\end{array}$ & $\begin{array}{l}\infty \\
\stackrel{1}{n}\end{array}$ & $\frac{N}{N}$ & $\vec{m}$ & $\stackrel{n}{n}$ & 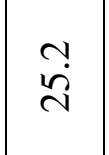 & రి & $\stackrel{\nabla}{\sim}$ & ñ & $\begin{array}{l}0 \\
\dot{q}\end{array}$ & $\stackrel{+}{\stackrel{+}{d}}$ & $\begin{array}{l}\infty \\
\stackrel{\sim}{d}\end{array}$ & $\stackrel{\Xi}{\sim}$ \\
\hline $\begin{array}{l}x \\
\hat{a} \\
\frac{1}{3}\end{array}$ & $\begin{array}{l}0 \\
\text { ஸे }\end{array}$ & त̃ & $\stackrel{n}{n}$ & $\vec{n}$ & $\stackrel{\nabla}{\stackrel{\nabla}{~}}$ & $\begin{array}{l}\infty \\
\infty \\
n\end{array}$ & $\begin{array}{l}\infty \\
\ddot{N}\end{array}$ & 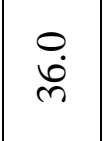 & aे & $\begin{array}{l}\infty \\
\dot{\sim}\end{array}$ & 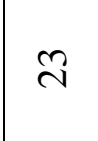 & $\vec{\sim}$ \\
\hline$\stackrel{\infty}{\circ}$ & $\stackrel{\infty}{\dot{m}}$ & $\hat{\vartheta}$ & $\stackrel{\nabla}{\stackrel{\sim}{r}}$ & $\begin{array}{l}\infty \\
\dot{m}\end{array}$ & $\frac{a}{\sim}$ & $\begin{array}{l}0 \\
\stackrel{0}{0}\end{array}$ & $\hat{a}$ & $\stackrel{m}{n}$ & ஓें & $\hat{\grave{d}}$ & $\stackrel{\nabla}{\partial}$ & $\frac{N}{\sim}$ \\
\hline 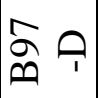 & $\frac{0}{m}$ & $\ddot{\vec{N}}$ & ñ & $\stackrel{0}{m}$ & $\stackrel{0}{\vec{n}}$ & $\begin{array}{l}0 \\
\dot{0}\end{array}$ & $\dddot{n}$ & $\stackrel{\infty}{\dot{m}}$ & $\begin{array}{l}\infty \\
\infty \\
\infty\end{array}$ & $\ddot{i}$ & $\stackrel{1}{2}$ & $\dot{i}$ \\
\hline 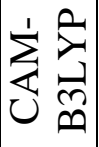 & ن் & $\stackrel{\nabla}{\ddot{N}}$ & $\stackrel{\infty}{\infty}$ & กิ & $\stackrel{m}{\sim}$ & $\begin{array}{l}0 \\
\dot{m}\end{array}$ & $\stackrel{\nabla}{\partial}$ & $\dot{\vec{n}}$ & $\begin{array}{l}0 \\
\text { n }\end{array}$ & $\stackrel{\circ}{\stackrel{\leftrightarrow}{~}}$ & $\stackrel{m}{\infty}$ & $\ddot{\grave{d}}$ \\
\hline $\begin{array}{l}\vec{\partial} \\
\ddot{n}\end{array}$ & $\begin{array}{l}0 \\
\stackrel{\infty}{N}\end{array}$ & $\hat{a}$ & ڤ̊. & ஜே. & $\stackrel{\infty}{\perp}$ & ñ & in & 穴 & $\dot{n}$ & $\stackrel{n}{0}$ & $\ddot{n}$ & $\begin{array}{l}0 \\
0\end{array}$ \\
\hline $\bar{a}$ & $\vec{\infty}$ & $\begin{array}{l}\infty \\
\stackrel{0}{0}\end{array}$ & ํํ & $\dot{\dot{q}}$ & $\begin{array}{l}0 \\
i n\end{array}$ & $\begin{array}{l}\stackrel{\leftrightarrow}{\dot{J}} \\
\stackrel{\sim}{f}\end{array}$ & $\stackrel{n}{\sim}$ & $\stackrel{\circ}{\stackrel{\leftrightarrow}{\sim}}$ & $\begin{array}{l}0 \\
\dot{+}\end{array}$ & $\stackrel{n}{\sim}$ & $\vec{\sim}$ & $\stackrel{\sim}{\sim}$ \\
\hline$\frac{\Gamma}{n}$ & $\frac{a}{n}$ & $\stackrel{n}{\sim}$ & $\begin{array}{l}\infty \\
\infty \\
\infty\end{array}$ & बें & $\stackrel{\sim}{\dot{d}}$ & $\stackrel{\Re}{\sim}$ & 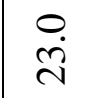 & $\frac{9}{\dot{y}}$ & $\stackrel{r}{r}$ & 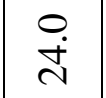 & $\begin{array}{l}\infty \\
\stackrel{i}{ }\end{array}$ & $\stackrel{n}{\sim}$ \\
\hline $\begin{array}{l}\infty \\
\infty \\
\infty\end{array}$ & $\begin{array}{l}\infty \\
\ddot{n}\end{array}$ & $\underset{⿱}{\stackrel{\Delta}{J}}$ & 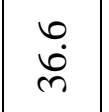 & $\frac{\infty}{m}$ & $\stackrel{+}{\Delta}$ & $\stackrel{\circ}{\stackrel{+}{+}}$ & $\stackrel{0}{\dot{0}}$ & ñ & $\stackrel{\vec{r}}{\dot{\nabla}}$ & $\frac{n}{\sim}$ & ণ্ণ & $\frac{\infty}{\sim}$ \\
\hline 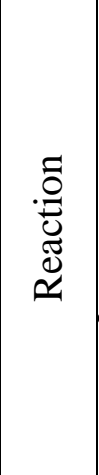 & 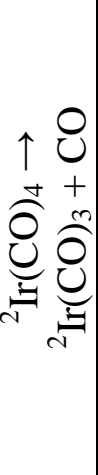 & 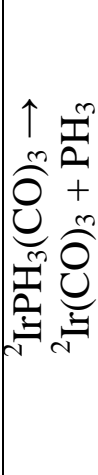 & 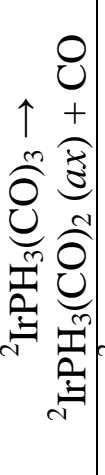 & 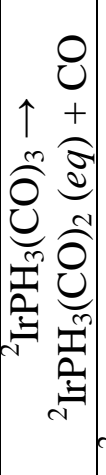 & 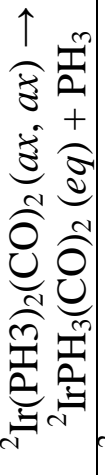 & 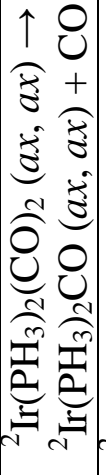 & 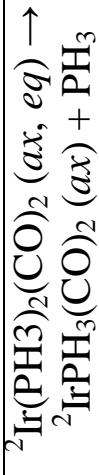 & 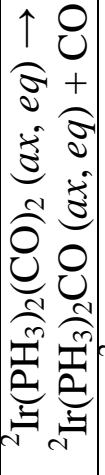 & 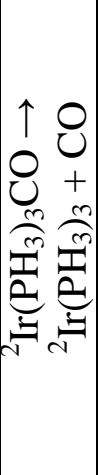 & 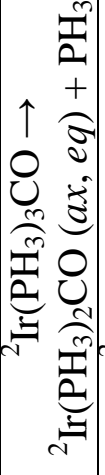 & 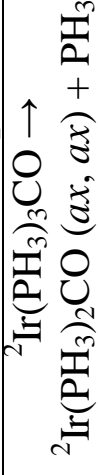 & 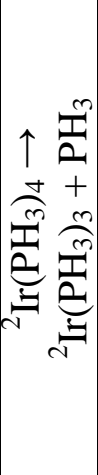 \\
\hline
\end{tabular}


the bulk of the phosphine ligand. Substitution of a second $\mathrm{PH}_{3}$ leads to $\operatorname{Ir}\left(\mathrm{PH}_{3}\right)_{2} \mathrm{CO}$, which has two isomers, one with the $\mathrm{PH}_{3}$ ligands cis to each other in an axial and an equatorial position (1e) and one with the $\mathrm{PH}_{3}$ groups trans to each other, both in axial positions (1d). Structure 1d has a lower energy by $1.3 \mathrm{kcal} / \mathrm{mol}$ than $1 \mathrm{e}$, showing that axial substitution isomers are again favored. $\operatorname{Ir}(\mathrm{CO})_{4}$ has $\mathrm{D}_{2 \mathrm{~d}}$ symmetry, falling between planar $\mathrm{D}_{4 \mathrm{~h}}$ and tetrahedral $\mathrm{T}_{\mathrm{d}}$ structures. Substitution of a $\mathrm{CO}$ bv $\mathrm{PH}_{2}$ leads to $\mathrm{IrPH}_{3}(\mathrm{CO})_{3}(\mathbf{1 h})$ and lowers the symmetry to $\mathrm{C}_{\mathrm{s}}$. Substitution of a second $\mathrm{PH}_{3}$ can occur either trans (two $a x, \mathbf{1 i}$ ) or cis (one $a x$ and one $e q, \mathbf{1 j}$ ). Structure 1i leads to the lower-energy structure by $1.4 \mathrm{kcal} / \mathrm{mol}$, essentially the same energy difference as found in $\operatorname{Ir}\left(\mathrm{PH}_{3}\right)_{2} \mathrm{CO}$.

The results for the LDEs of the $\operatorname{Ir}\left(\mathrm{PH}_{3}\right)_{y}(\mathrm{CO})_{z}$ complexes can be explained in terms of the basic Dewar-Chatt-Duncanson model of $\sigma$-electron donation from the ligand to the metal coupled with $\pi$-back-bonding to the ligand unoccupied orbitals to explain the LDEs.[78,79] This model has been expanded by considering other orbitals beyond the frontier molecular orbitals.[80] When a $\mathrm{PH}_{3}$, a stronger $\sigma$-electron donor than carbonyl, is substituted for $\mathrm{CO}$ on an iridium carbonyl cluster, more electrons occupy the Ir $\mathrm{d}$ orbitals. The increase in electron density on the $\mathrm{Ir}$ core can lead to more $\pi$-back-bonding to the $\mathrm{CO} \pi^{*}$ orbitals and to the $\mathrm{P}-\mathrm{H} \sigma^{*}$ orbitals. The Ir-C bond lengths decrease and the $\mathrm{C}-\mathrm{O}$ bond length increases with a concomitant decrease in the $\mathrm{CO}$ stretching frequency as $\mathrm{PH}_{3}$ groups are added to a single $\mathrm{Ir}$, as the occupation of the $\pi^{*}$ orbitals weakens the $\mathrm{C}-\mathrm{O}$ bonds. The $\mathrm{P}-\mathrm{H} \sigma^{*}$ orbitals are significantly less energetically available than the $\mathrm{CO} \pi^{*}$ orbitals, and so $\mathrm{PH}_{3}$ is a weaker electron accepter than $\mathrm{CO}$. Back-bonding of the metal $\mathrm{d}$ orbitals to these $\mathrm{P}-\mathrm{H} \sigma^{*}$ orbitals can increase on binding a second $\mathrm{PH}_{3}$ as a consequence of the increased electron density on the metal because of binding the first $\mathrm{PH}_{3}$. However, the increase in electron density on the metal can repel the lone-pair electrons on $\mathrm{PH}_{3}$, which form the donor $\sigma$-bonds, thus counteracting the back-bonding. The back-bonding results in competition between the two ligands trans to each other for these electrons because of the shape of the $d$ orbitals. In $\operatorname{Ir}\left(\mathrm{PH}_{3}\right)_{\mathrm{y}}(\mathrm{CO})_{\mathrm{z}}$ molecules, carbonyls are stronger $\pi$-electron accepters than $\mathrm{PH}_{3}$ ligands. Thus, a ligand-metal bond is weakened when there is a carbonyl instead of $\mathrm{PH}_{3}$ trans to it, and carbonyls are considered to have a stronger trans effect.

The calculated NBO charge,[81,82,83,84,85,] natural Ir 5d orbital population, $\mathrm{C}-\mathrm{O}$ stretching frequency, bond length, and LDEs of $\mathrm{CO}$ and $\mathrm{PH}_{3}$ ligands of $\operatorname{Ir}\left(\mathrm{PH}_{3}\right)_{y}(\mathrm{CO})_{z}$ are listed in Table 3. For $\mathrm{IrL}_{3}$, the expected trend as stated above is evident. As more carbonyls are replaced by phosphines, the $5 \mathrm{~d}$ orbital population and charge on the iridium atom increase, concomitant with stronger $\mathrm{Ir}-\mathrm{C}$ bonds and weaker $\mathrm{C}-\mathrm{O}$ bonds. Because the product linear $\mathrm{IrL}_{2}$ is of higher energy, the eq CO ligands have the highest LDEs, 51 to $59 \mathrm{kcal} / \mathrm{mol}$. Because eq $\mathrm{CO}$ ligands in $\mathrm{IrL}_{3}$ do not have a ligand trans to them, the $8 \mathrm{kcal} / \mathrm{mol}$ energy difference suggests that electron density is transferred from ligands cis to the eq $\mathrm{CO}$ through $\sigma$-donation. The difference in the LDEs between ax COs in $1 \mathrm{a}$ and $1 \mathrm{e}$ is $11 \mathrm{kcal} / \mathrm{mol}$ and suggests that transfer of electrons to the trans position is more efficient than to the cis. More efficient electron transfer, a smaller trans effect, and a smaller steric effect make 1b the lower energy isomer.

The trend of the $\mathrm{PH}_{3}$ LDEs is more complicated. From $\mathbf{1 b}$ to $\mathbf{1 f}$, the energy differences in both eq $\mathrm{PH}_{3}$ and ax $\mathrm{PH}_{3}$ LDEs are less than $3 \mathrm{kcal} / \mathrm{mol}$. In most cases, the strength of the bonds is reflected by bond lengths and LDEs, but this may not 


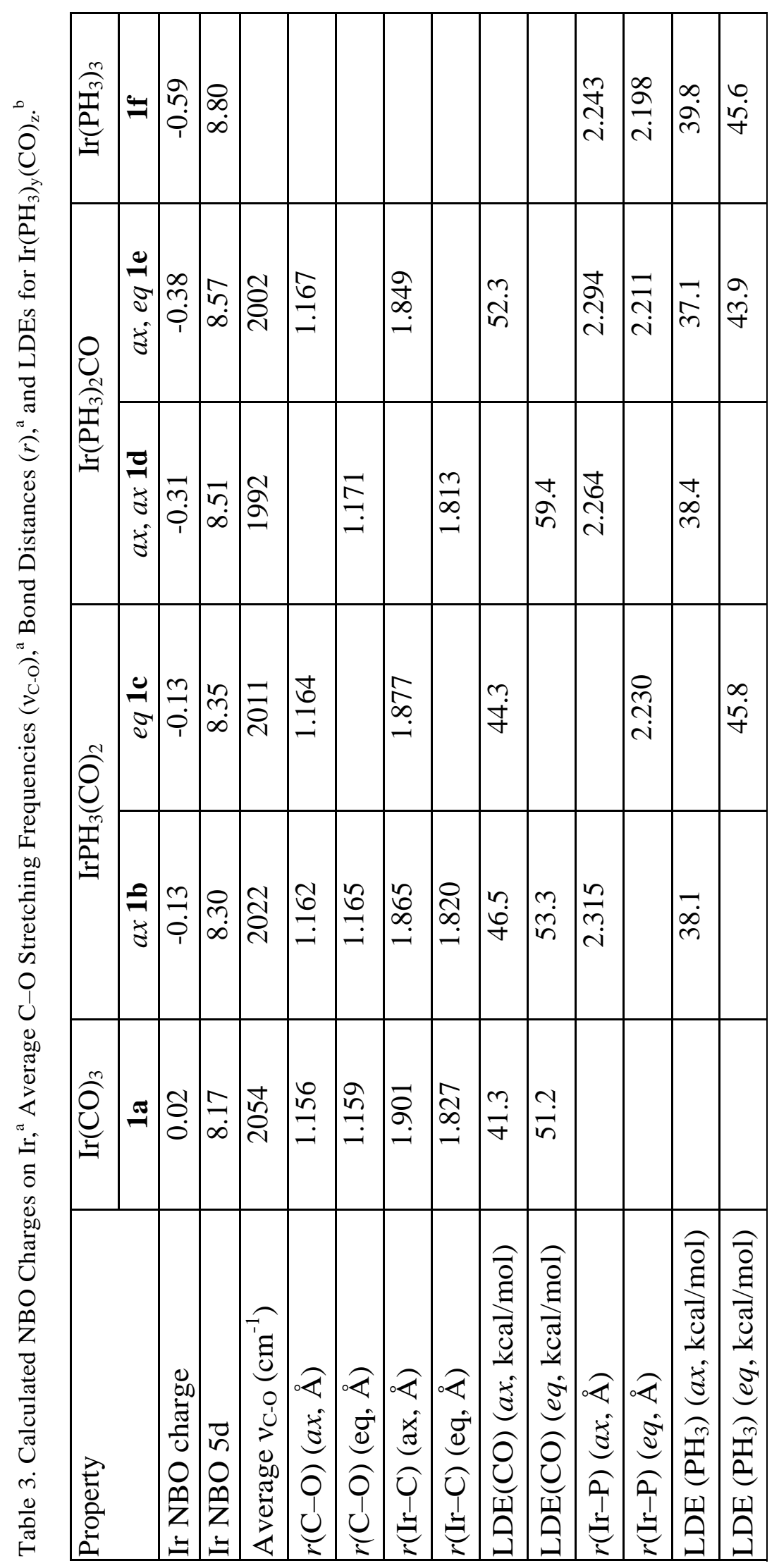




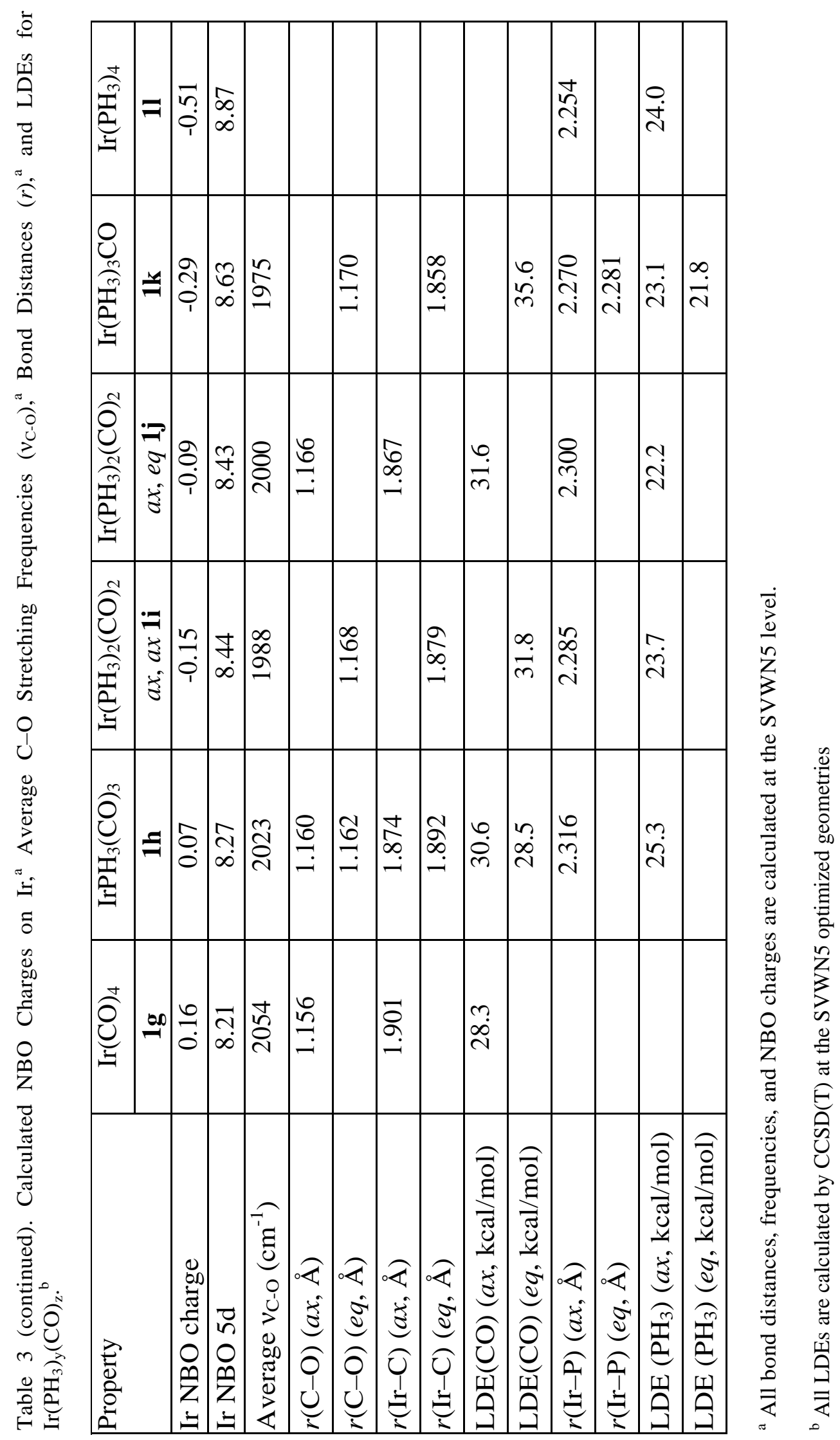


always be true.[86] The carbonyl cis to the ax (eq) $\mathrm{PH}_{3}$ is replaced by $\mathrm{PH}_{3}$ to form 1e from $1 \mathbf{b}$ and 1c. The new $\mathrm{PH}_{3}$ increases the electron density on the Ir atom, which strengthens the $\pi$-back-bonding and increases repulsion between the two $\sigma$-electrons from each $\mathrm{PH}_{3}$. The decrease in the $\mathrm{Ir}-\mathrm{P}$ bond lengths suggests that the iridium and $\mathrm{PH}_{3}$ orbitals have better overlap, but the decrease in the LDE of the ax (eq) $\mathrm{PH}_{3}$ shows that it is easier to dissociate. It is likely that the decrease in LDE is a consequence of the product $\mathrm{IrL}_{2}$ becoming of lower energy, i.e., more stable. Substitution from 1e to generate $1 \mathbf{f}$ (and from $\mathbf{1 b}$ to $1 \mathbf{d}$ ) leads to the replacement of the carbonyl trans to an $a x$ $\mathrm{PH}_{3}$. In this case, the Ir-P bond length decreases, and there is an increase in the LDE. In this case, the product $\operatorname{IrL}_{2}$ is higher in energy, giving a higher LDE.

For $\mathrm{IrL}_{4}$ clusters, we predict that the LDEs are $\sim 10$ to $20 \mathrm{kcal} / \mathrm{mol}$ less than that of $\mathrm{IrL}_{3}$, whether a $\mathrm{PH}_{3}$ or a $\mathrm{CO}$ is added, and there are no remarkable changes in the $a x$ bond lengths and frequencies. Different from the almost planar structure of $\mathrm{IrL}_{3}, \mathrm{IrL}_{4}$ isomers are distorted with dihedral angles $\angle \mathrm{L}(a x)-\mathrm{L}(e q)-\mathrm{Ir}-\mathrm{L}(a x)$ of $140^{\circ}$ to $147^{\circ}$. This result suggests there are more steric interactions in $\mathrm{IrL}_{4}$ isomers leading to the distortion and decreasing the LDEs. Analogous to the trend in the $\mathrm{IrL}_{3} \mathrm{LDEs}$, the LDEs of the carbonyls increase as more $\mathrm{CO}$ ligands are replaced by $\mathrm{PH}_{3}$. The difference is less than in $\mathrm{IrL}_{3}$, which is likely best attributed to the smaller LDEs. The LDEs of $\mathrm{PH}_{3}$ show a small decrease of $3 \mathrm{kcal} / \mathrm{mol}$ from $\mathbf{1 h}$ to $\mathbf{1 i} / \mathbf{1 j}$ to $\mathbf{1 k}$ for $\mathrm{PH}_{3}$ trans to $\mathrm{CO}$ and a smaller decrease of $<1 \mathrm{kcal} / \mathrm{mol}$ for $\mathrm{PH}_{3}$ trans to $\mathrm{PH}_{3}$. Replacement of $\mathrm{CO}$ by $\mathrm{PH}_{3}$ to generate $1 \mathbf{k}$ from $1 \mathbf{i}$ (and $1 \mathbf{k}$ from $\mathbf{1 j}$ ), leads to trends similar to those found for $\mathrm{IrL}_{3}$. When the carbonyl cis to the $\mathrm{PH}_{3}$ is replaced, both the Ir-P bond length and the LDE of the $\mathrm{PH}_{3}$ decrease. If the carbonyl trans to the $\mathrm{PH}_{3}$ is replaced (converting $\mathbf{1 j}$ to $\mathbf{1 k}$ ), the Ir-P bond length decreases and the $\mathrm{PH}_{3} \mathrm{LDE}$ increases.

\section{2 $\operatorname{Ir}_{2}\left(\mathrm{PH}_{3}\right)_{\mathrm{y}}(\mathrm{CO})_{\mathrm{z}}$ structures, relative energies, and ligand dissociation energies.}

$\mathrm{Ir}_{2}(\mathrm{CO})_{8}$ has three low-energy isomers, [51] which we previously optimized, and three fundamental structures of $\operatorname{Ir}_{2}\left(\mathrm{PH}_{3}\right)_{\mathrm{y}}(\mathrm{CO})_{\mathrm{z}}$ are predicted: $\mathrm{C}_{2 \mathrm{v}}\left(\mathrm{C}_{\mathrm{s}}\right), \mathrm{C}_{2}$, and $\mathrm{D}_{3 \mathrm{~d}}$ (Figure 3). The lowest energy of $\operatorname{Ir}_{2}(\mathrm{CO})_{8}$ has a Ir-Ir bond consistent with the matrix observations of Ozin.[27] The bond distances are given in the Supporting Information, and relative energies of the isomers and derivatives are listed in Table 4 . The $\mathrm{C}_{2 \mathrm{v}}\left(\mathrm{C}_{\mathrm{s}}\right)$ structure has two bridging CO ligands above two Ir atoms $(\mathbf{2 a}, \mathbf{2 d}, \mathbf{2 g}, \mathbf{2 h}, \mathbf{2 j}, \mathbf{2 l}, \mathbf{2 n}$, $\mathbf{2 p}, \mathbf{2 q}$, and $\mathbf{2 s}$ ) and results in shorter $\mathrm{Ir}-\mathrm{Ir}$ bonds than in the $\mathrm{C}_{2}$ and $\mathrm{D}_{3 \mathrm{~d}}$ structures. The Ir-C (bridging $=$ bri) bond lengths are usually greater than other Ir-C (ter $=$ terminal) bond distances by $0.1-0.2 \AA$. As one bridging CO is dissociated (4 and 12), the two ax ligands on that side and the other bridging $\mathrm{CO}$ move towards the same plane $(\mathbf{2} \mathrm{g}$ and 2p). As one terminal $\mathrm{CO}$ is dissociated, the $\mathrm{C}_{2 \mathrm{v}}$ isomer transitions to a structure with the two remaining ligands more nearly equatorial $\left(\mathbf{2 a}, \mathbf{2 h}\right.$ and $\mathbf{2 q}$ ); the $\mathrm{C}_{\mathrm{s}}$ isomer

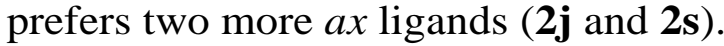

In the $\mathrm{C}_{2}$ structure, the $\mathrm{C}_{2}$ axis is perpendicular to the $\mathrm{Ir}-\mathrm{Ir}$ bond. Each Ir atom binds four ligands, with two closer to the other Ir atom and two farther from the other Ir atom. The Ir-C bond length depends on the carbonyl's direction; the CO ligands that are closer to the other Ir atom have a longer $\mathrm{Ir}-\mathrm{C}$ bond than the ones that face away from the other Ir atom. The Ir-C bonds along the $\mathrm{Ir}-\mathrm{Ir}$ bond axis (2b and $\mathbf{2 i}$ ) are always the shortest. For example, the average "farther" and "closer" Ir-C bond lengths are 1.887 and $1.900 \AA$ in $\mathbf{2 i}$, and 1.874 and $1.899 \AA$ in $\mathbf{2 m}$. Usually a $\mathrm{C}_{\mathrm{s}}$ structure is 
formed as one ligand is dissociated ( $\mathbf{2 b}$ and $\mathbf{2} \mathbf{i}$ ), except for $\mathbf{1 4}$, which has the same product as $\mathbf{1 3}$.

There are two staggered trigonal planar $\operatorname{Ir}(\mathrm{CO})_{3}$ moieties in the $\mathrm{D}_{3 \mathrm{~d}}$ structure of $\mathrm{Ir}_{2}(\mathrm{CO})_{8}$, and the other two ligands bind to each Ir atom along the $\mathrm{C}_{3}$ axis. Analogous to the $\mathrm{C}_{2}$ isomers, the ax $\mathrm{Ir}-\mathrm{C}$ bonds $(1.882 \AA$ ) are shorter than the $\mathrm{Ir}-\mathrm{C}$ bonds (1.906 $\AA$ ) in the two $\operatorname{Ir}(\mathrm{CO})_{3}$ moieties. [51] The $\mathrm{D}_{3 \mathrm{~d}}$ structure does not change much as one ligand is dissociated or replaced.

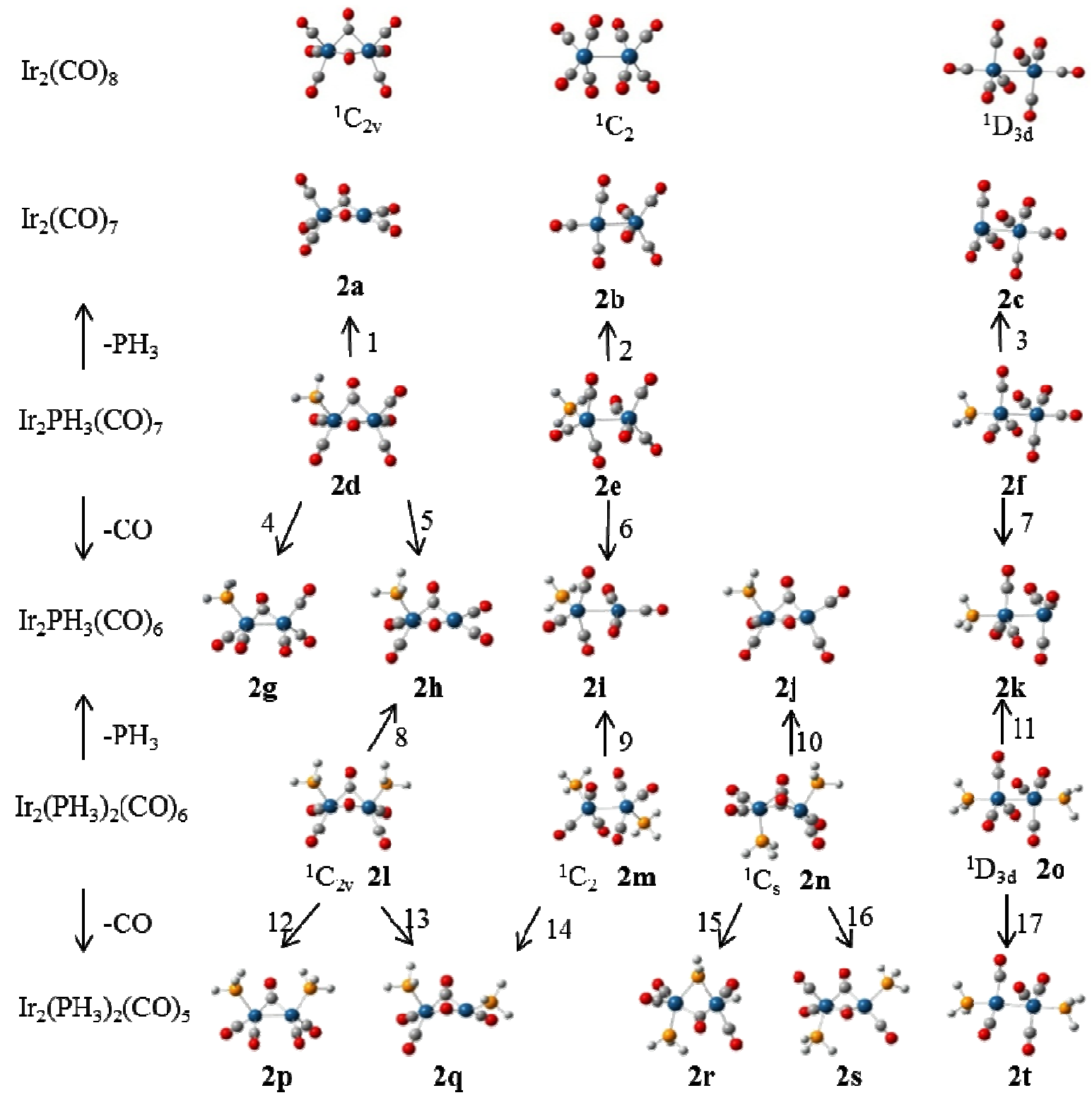

Figure 3: Optimized geometries of low-lying $\operatorname{Ir}_{2}\left(\mathrm{PH}_{3}\right)_{y}(\mathrm{CO})_{z}$ isomers. 


\begin{tabular}{|c|c|c|c|c|c|c|c|c|c|c|c|c|c|c|c|c|c|c|c|c|c|}
\hline$\stackrel{\ominus}{\ominus}$ & 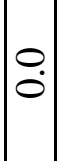 & $\stackrel{\sim}{=}$ & $\begin{array}{l}0 \\
\infty \\
\sim \\
\sim\end{array}$ & $\stackrel{0}{0}$ & $\overrightarrow{\mathrm{N}}$ & r. & $\dot{i}$ & 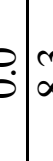 & $\begin{array}{c}? \\
\infty\end{array}$ & $\begin{array}{l}0 \\
0 \\
0\end{array}$ & $\begin{array}{l}0 \\
\dot{J}\end{array}$ & $\left|\begin{array}{c}0 \\
0 \\
0 \\
\end{array}\right|$ & $\stackrel{0}{0}$ & $\begin{array}{l}0 \\
0\end{array}$ & $\stackrel{N}{-1}$ & $\begin{array}{l}\text { İ } \\
\pm\end{array}$ & : & $\begin{array}{l}\infty \\
\sim \\
\sim \\
1\end{array}$ & $\hat{\infty}$ & $\begin{array}{l}\infty \\
\infty \\
-1\end{array}$ & $\vec{\curvearrowright}$ \\
\hline$\stackrel{\Sigma}{\stackrel{\Sigma}{\Sigma}}$ & : & $\vec{r}$ & $\begin{array}{l}0 \\
\grave{2}\end{array}$ & $\stackrel{0}{\circ}$ & 9 & mे & $\dot{i}$ & $\dot{\vec{b}} \cdot \vec{c}$ & $\stackrel{0}{\dot{m}}$ & $\stackrel{?}{+}$ & $\mid \begin{array}{l}0 \\
- \\
-\end{array}$ & $\begin{array}{l}\infty \\
\grave{\lambda} \\
\vdots\end{array}$ & $\begin{array}{l}0 \\
0\end{array}$ & $\mid \begin{array}{l}0 \\
0 \\
1\end{array}$ & $\vec{\sim}$ & $\vec{a}$ & 0 & $\begin{array}{l}\stackrel{\dot{J}}{\Xi} \\
\end{array}$ & $\begin{array}{l}\checkmark \\
\stackrel{v}{.}\end{array}$ & Iי & $\begin{array}{c}\infty \\
\dot{\sim}\end{array}$ \\
\hline$\stackrel{8}{\stackrel{2}{z}}$ & $\stackrel{0}{0}$ & 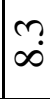 & $\stackrel{\nabla}{\stackrel{\theta}{0}}$ & $\stackrel{0}{0}$ & $\stackrel{0}{0}$ & $\stackrel{\circ}{0}$ & $\dot{b}$ & 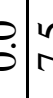 & $?$ & $\begin{array}{l}0 \\
0 \\
0\end{array}$ & $\begin{array}{l}\nabla \\
\sigma \\
\sigma\end{array}$ & $\begin{array}{l}\vec{i} \\
\text { mे }\end{array}$ & $\stackrel{0}{0}$ & $\begin{array}{l}0 \\
\dot{r}\end{array}$ & - & $\underline{n}$ & $\stackrel{0}{0}$ & $\begin{array}{l}n \\
n\end{array}$ & $\overrightarrow{0}$ & $\stackrel{\nabla}{\sim}$ & $\begin{array}{c}- \\
\infty \\
-1\end{array}$ \\
\hline 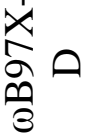 & 0 & 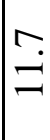 & ฉ̊ & $\stackrel{0}{0}$ & $\stackrel{0}{-}$ & 9 & $\therefore$ & $\dot{0} \cdot \mid 2$ & $\begin{array}{c}\Upsilon \\
\infty \\
\infty\end{array}$ & $\hat{a}$ & 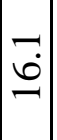 & $\frac{n}{n}$ & $\stackrel{0}{0}$ & $\stackrel{\sim}{\sim}$ & 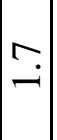 & {$\left[\begin{array}{l}n \\
0 \\
0\end{array}\right.$} & 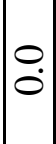 & $\begin{array}{l}n \\
\\
\end{array}$ & $\stackrel{5}{\check{I}}$ & $\begin{array}{l}0 \\
\\
\end{array}$ & $\vec{d}$ \\
\hline $\begin{array}{l}x \\
\hat{\sigma} \\
\frac{\hat{\sigma}}{3}\end{array}$ & : & $\stackrel{\sim}{N}$ & $\stackrel{\overbrace{}}{\Omega}$ & 0 & $\stackrel{\sim}{\sim}$ & $\begin{array}{l}0 \\
\text { i }\end{array}$ & $\begin{array}{l}i \\
\mathrm{i}\end{array}$ & $\dot{0} \cdot 0$ & ה!. & $\ddot{0}$ & $\left|\begin{array}{c}\infty \\
0 \\
-0\end{array}\right|$ & $\frac{a}{m}$ & $\begin{array}{l}0 \\
0\end{array}$ & $\dot{a}$ & $\stackrel{n}{-}$ & 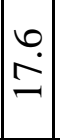 & $\begin{array}{l}0 \\
0\end{array}$ & $\stackrel{\Omega}{\tilde{N}}$ & $\tilde{a}$ & $\begin{array}{l}a \\
\infty\end{array} \mid$ & $\begin{array}{l}\text { フุ. } \\
\end{array}$ \\
\hline$\stackrel{\infty}{\curvearrowright}$ & ○. & 号 & กุ & $\stackrel{0}{0}$ & بै & $?$ & $?$ & 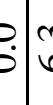 & $\begin{array}{l}3 \\
0\end{array}$ & $\begin{array}{c}1 \\
\infty\end{array}$ & $\stackrel{+}{\stackrel{+}{ \pm}}$ & 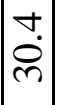 & $\stackrel{0}{0}$ & $\dot{a}$ & $\underset{0}{0}$ & $\because$ & $\begin{array}{l}0 \\
0\end{array}$ & ñ & $\begin{array}{l}\infty \\
\dot{\sim} \\
-\end{array}$ & 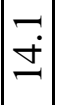 & $\begin{array}{l}0 \\
\grave{d} \\
\stackrel{\nu}{0}\end{array}$ \\
\hline 官 & 0 & 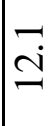 & $\begin{array}{l}0 \\
\dot{\sim}\end{array}$ & $\stackrel{0}{\circ}$ & $\vec{i}$ & テ & $\stackrel{0}{1}$ & كا. & $\overrightarrow{6}$ & $\begin{array}{l}0 \\
0 \\
0\end{array} \mid$ & $\begin{array}{l}\dot{\sim} \\
\dot{\sigma}\end{array}$ & $\begin{array}{l}0 \\
\dot{2} \\
\lambda\end{array}$ & $\stackrel{0}{0}$ & 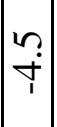 & $\frac{N}{1}$ & $\begin{array}{c}0 \\
\dot{2}\end{array}$ & $\stackrel{0}{0}$ & $\overrightarrow{+}$ & $\begin{array}{l}\infty \\
\dot{0} \\
\dot{0}\end{array}$ & $\stackrel{?}{\beth}$ & $\stackrel{2}{2}$ \\
\hline$\sum_{0}^{\infty} \frac{n}{\pi}$ & 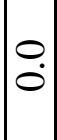 & $\stackrel{n}{=}$ & 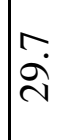 & $\stackrel{0}{0}$ & $\infty$ & r & $\begin{array}{l}i \\
i\end{array}$ & $\dot{p} \cdot \vec{c}$ & $\infty$ & $\begin{array}{l}\infty \\
\alpha \\
\alpha\end{array} \mid$ & $\begin{array}{l}n \\
0 \\
-\end{array}$ & $\frac{0}{n}$ & $\begin{array}{l}0 \\
0\end{array}$ & $\begin{array}{l}0 \\
0\end{array}$ & 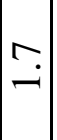 & $\stackrel{-}{二}$ & $\stackrel{0}{0}$ & $\begin{array}{l}0 \\
0 \\
0 \\
0\end{array}$ & $\begin{array}{l}0 \\
1 \\
-1\end{array}$ & $\underline{0}$ & $\vec{\sim}$ \\
\hline 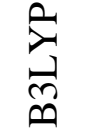 & $\begin{array}{l}0 \\
0\end{array}$ & $\ddot{0}$ & กุ & $\stackrel{0}{\circ}$ & $\stackrel{n}{ }$ & $\cong$ & 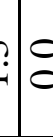 & 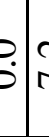 & Na & $\begin{array}{l}a \\
\infty\end{array}$ & $\ddot{n}$ & $\begin{array}{c}\dot{0} \\
\dot{m}\end{array}$ & 0 & $\overline{0}$ & $\begin{array}{l}\infty \\
0 \\
0\end{array}$ & {$\left[\begin{array}{l}n \\
n \\
n\end{array}\right.$} & $\stackrel{0}{0}$ & $\left|\begin{array}{l}0 \\
0 \\
0\end{array}\right|$ & $\begin{array}{c}0 \\
\dot{1} \\
1\end{array} \mid$ & $\mid \begin{array}{c}\simeq \\
\end{array}$ & $\underset{\infty}{0}$ \\
\hline $\bar{a}^{\bar{a}}$ & $\begin{array}{l}0 \\
0\end{array}$ & 6 & 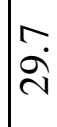 & $\stackrel{0}{\circ}$ & $\tilde{r}$ & F & $: 0$ & ¿ & - & $\vec{\nabla}$ & à & 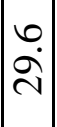 & $\begin{array}{l}0 \\
0\end{array}$ & $\begin{array}{l}\infty \\
\dot{r}\end{array}$ & $\begin{array}{l}0 \\
ن \\
ن\end{array} \mid$ & 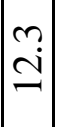 & $\stackrel{0}{0}$ & $\begin{array}{l}a \\
\end{array}$ & 苂 & $\stackrel{n}{\sim}$ & $\begin{array}{l}\infty \\
\infty \\
\infty\end{array} \mid$ \\
\hline$\frac{\omega}{a}$ & O̊. & 0. & $\infty$ & $\stackrel{0}{0}$ & $\underset{i}{r}$ & ־ & $: 0$ & $\stackrel{P}{\circ}$ & $\ddot{0}$ & $\dot{m}$ & $\begin{array}{l}a \\
\infty\end{array} \mid$ & 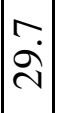 & $\begin{array}{l}0 \\
0\end{array}$ & $\begin{array}{l}0 \\
\dot{\nabla}\end{array}$ & $\vec{\sim}$ & 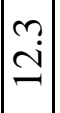 & $\stackrel{0}{0}$ & $\begin{array}{l}+ \\
\dot{\sim} \\
\dot{\sigma}\end{array}$ & $\stackrel{\infty}{=}$ & $\stackrel{\sim}{\mathrm{i}}$ & $\begin{array}{l}0 \\
0 \\
0\end{array}$ \\
\hline $\begin{array}{l}0 \\
\infty \\
\infty \\
\infty\end{array}$ & $\stackrel{0}{0}$ & זי & 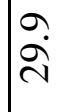 & $\stackrel{0}{0}$ & $\underset{\sim}{\sim}$ & Oे & $\dot{b}$ & $\stackrel{5}{\circ}$ & Fi & 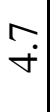 & $\stackrel{+}{\circ}$ & 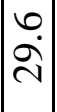 & $\stackrel{0}{0}$ & $\overrightarrow{\dot{m}}$ & $\stackrel{\sim}{\sim}$ & $\begin{array}{l}0 \\
i \\
\end{array}$ & $\stackrel{0}{\circ}$ & $\begin{array}{c}0 \\
\dot{1} \\
-\end{array} \mid$ & $\stackrel{n}{=}$ & $\begin{array}{l}\stackrel{\sim}{\sim} \\
\stackrel{i}{*}\end{array}$ & $\begin{array}{c}+ \\
\infty \\
-\end{array}$ \\
\hline$\frac{\frac{0}{J}}{\frac{d}{0}}$ & 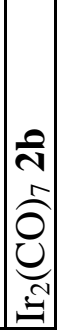 & 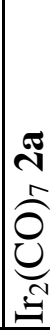 & 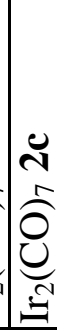 & 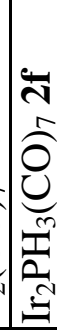 & 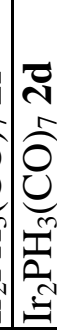 & $=$ & 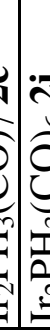 & 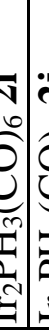 & 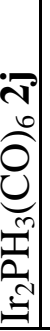 & 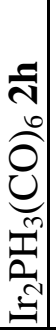 & 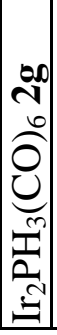 & 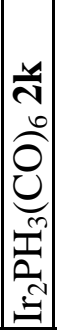 & 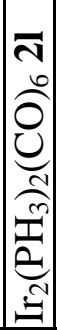 & 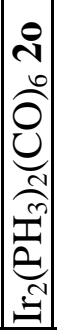 & 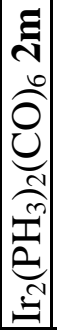 & 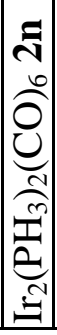 & 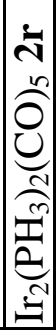 & 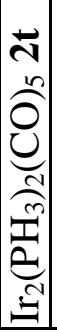 & 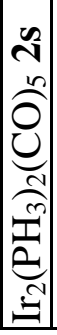 & 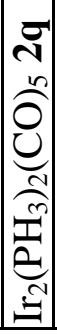 & 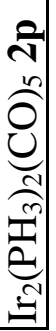 \\
\hline
\end{tabular}


Relative energies of the $\operatorname{Ir}_{2}\left(\mathrm{PH}_{3}\right)_{\mathrm{y}}(\mathrm{CO})_{\mathrm{z}}$ isomers are quite different between the coordinatively saturated clusters $\left(\mathrm{Ir}_{2} \mathrm{PH}_{3}(\mathrm{CO})_{7}\right.$ and $\left.\mathrm{Ir}_{2}\left(\mathrm{PH}_{3}\right)_{2}(\mathrm{CO})_{6}\right)$ and the unsaturated clusters $\left(\operatorname{Ir}_{2}(\mathrm{CO})_{7}, \mathrm{Ir}_{2} \mathrm{PH}_{3}(\mathrm{CO})_{6}\right.$, and $\left.\mathrm{Ir}_{2}\left(\mathrm{PH}_{3}\right)_{2}(\mathrm{CO})_{5}\right)$. Among the saturated clusters, the $\mathrm{C}_{2 \mathrm{v}}$ and $\mathrm{C}_{2}$ isomers have similar relative energies $\left(\mathbf{2 d}, \mathbf{2 e}\right.$, and $\mathbf{2 1}, \mathbf{2 m}, \Delta E_{\mathrm{rel}}<1.2$ $\mathrm{kcal} / \mathrm{mol}$ ). The higher energy isomer $2 \mathrm{n}$ has an imaginary frequency of $65 \mathrm{i} \mathrm{cm}^{-1}$ which is a rotation of the two $\mathrm{IrL}_{3}$ moieties about the $\mathrm{Ir}-\mathrm{Ir}$ axis. This structure is included because the compounds $\mathbf{2} \mathbf{j}, \mathbf{2 r}$, and $\mathbf{2 s}$ generated from it by a gain of $\mathrm{PH}_{3}$ or a loss of $\mathrm{CO}$ are minima. Among unsaturated clusters, the $\mathrm{C}_{2}$ structure derivatives $(\mathbf{2} \mathbf{b}$ and $\mathbf{2 i})$ are lower in energy by $\sim 10 \mathrm{kcal} / \mathrm{mol}$ than the $\mathrm{C}_{2 \mathrm{v}}$ structures. Among the $\mathrm{C}_{2 \mathrm{v}}$ derivatives formed when one terminal ligand is dissociated, the isomers with two more equatorial ligands, $\mathbf{2 h}$ and $\mathbf{2 q}$, have slightly higher energies than those with two more ax ligands, $\mathbf{2 j}$ and $\mathbf{2 s}$. The bridging-CO-dissociated $\mathrm{C}_{2 \mathrm{v}}$ derivatives $(\mathbf{2} \mathbf{g}$ and $\mathbf{2} \mathbf{p})$ are even higher in energy, by $\sim 5 \mathrm{kcal} / \mathrm{mol}$, than those with the terminal $\mathrm{CO}$ ligands dissociated. The relative energies of isomers with $\mathrm{D}_{3 \mathrm{~d}}$ symmetry depends on the nature of the two ligands on the Ir-Ir axis. If one of these two ligands is dissociated, the product becomes the one with highest energy among the isomers with higher relative energies (2c and 2k). However, the isomers with two such ligands are the lowest energy ones. Isomer $\mathbf{2 t}$ is an exception as $\mathbf{2 r}$ is lower in energy, but $\mathbf{2 t}$ does have a lower energy than any among $\mathbf{2 q}, \mathbf{2 p}$, and $\mathbf{2 s}$.

Dissociation of a bridging carbonyl from $2 \mathbf{n}$ to generate $\mathbf{2 r}$ (reaction 15) leads to a hydrogen atom transfer from a terminal $\mathrm{PH}_{3}$ to the $\mathrm{Ir}$ atom binding this $\mathrm{PH}_{3}$, and the $\mathrm{PH}_{2}$ now bridges the two Ir atoms. The product, $\mathbf{2 r}$, has a bri phosphido group occupying a similar position to the dissociated carbonyl. As discussed below, we find for the tetrairidium clusters that such hydrogen transfers often occur when a bridging carbonyl is dissociated, especially if there is a bri $\mathrm{PH}_{3}$ connecting to the same Ir atom, and that the products of such reactions always have much lower relative energies than other isomers.

The $\mathrm{Ir}_{2}\left(\mathrm{PH}_{3}\right)_{\mathrm{y}}(\mathrm{CO})_{\mathrm{z}} \mathrm{CO}$ and $\mathrm{PH}_{3}$ LDEs are listed in Table 5, and we readily conclude that (1) the ligands on the $\mathrm{Ir}-\mathrm{Ir}$ axis in the isomers with a $\mathrm{D}_{3 \mathrm{~d}}$ structure have the highest LDEs, nearly $50 \mathrm{kcal} / \mathrm{mol}$; (2) bridging carbonyls have the second highest LDEs, $~ 30 \mathrm{kcal} / \mathrm{mol}$; (3) the LDEs of terminal ligands in the $\mathrm{C}_{2 \mathrm{v}}$ structure are $\sim 25$ to $29 \mathrm{kcal} / \mathrm{mol}$; and (4) ligands in the $\mathrm{C}_{2}$ structure have the lowest LDEs, 15 to 17 $\mathrm{kcal} / \mathrm{mol}$. This set of results excludes reaction 15 , which involves hydrogen transfer from $\mathrm{PH}_{3}$ to Ir. The difference in the LDEs for all terminal ligands is less than 3 $\mathrm{kcal} / \mathrm{mol}$, and a similar range is found for the bridging ligands.

The influence of replacing $\mathrm{CO}$ by $\mathrm{PH}_{3}$ on the $\mathrm{Ir}-\mathrm{Ir}$ bond lengths and bond energies is given in Table 6. The results suggest that the increase in electron density on the Ir associated with the $\mathrm{CO}$ replacement leads to an increase in the Ir-Ir bond energy. The NBO charges on Ir do not change significantly on substitution of $\mathrm{CO}$ by $\mathrm{PH}_{3}$. The NBO charge on Ir that binds a $\mathrm{PH}_{3}$ in $\mathrm{Ir}_{2} \mathrm{PH}_{3}(\mathrm{CO})_{7}$ is the same as that of Ir in $\mathrm{Ir}_{2}\left(\mathrm{PH}_{3}\right)_{2}(\mathrm{CO})_{6}$, whereas that of the other Ir is the same as that of $\mathrm{Ir}$ in $\operatorname{Ir}_{2}(\mathrm{CO})_{8}$. The $\mathrm{Ir}-\mathrm{Ir}$ bond energy increases as $\mathrm{PH}_{3}$ is substituted for $\mathrm{CO}$. The two bridging $\mathrm{Ir}-\mathrm{C}$ bond lengths in $\mathrm{Ir}_{2} \mathrm{PH}_{3}(\mathrm{CO})_{7}$ differ, with one being shorter, as expected, and the other longer than that in $\mathrm{C}_{2 \mathrm{v}} \mathrm{Ir}_{2}(\mathrm{CO})_{8}$. The higher occupancy on the $\mathrm{C}-\mathrm{O}$ antibonding orbital resulting from increasing the electron density of the Ir atom that binds a phosphine 


\begin{tabular}{|c|c|c|c|c|c|c|c|c|c|c|c|c|c|c|c|c|c|}
\hline$\stackrel{\Xi}{\stackrel{\Xi}{己}}$ & $\begin{array}{l}0 \\
\infty \\
\stackrel{0}{0}\end{array}$ & - & $\vec{\infty}$ & 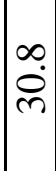 & $\begin{array}{l}\infty \\
\check{\lambda} \\
\sim\end{array}$ & $\begin{array}{l}0 \\
\dot{2} \\
-\end{array}$ & $\mid \begin{array}{c}0 \\
\infty \\
\dot{0}\end{array}$ & ন & $\mid \begin{array}{l}0 \\
0 \\
-\end{array}$ & $\stackrel{0}{0}$ & $\frac{n}{f}$ & $\begin{array}{c}n \\
m \\
m\end{array}$ & $\begin{array}{c}n \\
\infty \\
\infty \\
\end{array}$ & & $\stackrel{?}{m}:$ & $\dot{v}$ & \\
\hline$\stackrel{N}{\Sigma}$ & $\mid \begin{array}{l}n \\
0 \\
n \\
n\end{array}$ & $\begin{array}{l}0 \\
\infty \\
\stackrel{0}{0}\end{array} \mid$ & $\frac{n}{6}$ & $\frac{m}{m}$ & $\begin{array}{l}0 \\
\dot{m} \\
m\end{array}$ & $\begin{array}{l}\infty \\
\grave{\lambda}\end{array} \mid$ & $\frac{n}{6}$ & ma & $\begin{array}{l}0 \\
\hat{N}\end{array}$ & $\begin{array}{l}\sim \\
\stackrel{\sim}{\sim}\end{array}$ & $\begin{array}{l}0 \\
\dot{0}\end{array}$ & $\mid \begin{array}{c}r \\
\dot{v} \\
\tilde{c}\end{array}$ & $\begin{array}{l}a \\
\tilde{n} \\
\vdots\end{array}$ & m. & $\begin{array}{c}0 \\
0 \\
a\end{array}$ & $\begin{array}{l}\vec{v} \\
\dot{v}\end{array}$ & $\dot{n}$ \\
\hline$\stackrel{8}{\stackrel{2}{2}}$ & $\stackrel{-}{\infty}$ & $\stackrel{+}{\circ}$ & $\begin{array}{l}\hat{a} \\
\dot{q}\end{array}$ & $\begin{array}{l}n \\
\vdots \\
n \\
n\end{array}$ & $\mid \begin{array}{l}\infty \\
\vdots \\
0\end{array}$ & $\vec{c}$ & $\begin{array}{l}\infty \\
\dot{\gamma} \\
\gamma\end{array}$ & $\stackrel{?}{=}$ & $\vec{a}$ & $\stackrel{\sim}{\dot{\gamma}}$ & $\begin{array}{l}\infty \\
\infty \\
\tilde{m}\end{array}$ & $\mid \begin{array}{l}n \\
n \\
n\end{array}$ & 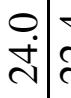 & 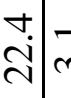 & $\dot{m}$ & $\dot{0}$ & t. \\
\hline $\begin{array}{l}\underset{\hat{\sigma}}{x} \\
\text { ô } \\
3\end{array}$ & $\mid \begin{array}{l}\nabla \\
\stackrel{0}{\sim} \\
\sim\end{array}$ & ?ִ & $\stackrel{r}{\dot{\sigma}}$ & $\begin{array}{l}0 \\
\dot{m}\end{array}$ & 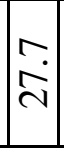 & $\stackrel{n}{=}$ & $\begin{array}{l}0 \\
\dot{0} \\
\end{array}$ & $\mid \begin{array}{l}\infty \\
\dot{0} \\
ن\end{array}$ & 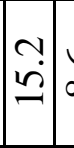 & $\left|\begin{array}{c}0 \\
\infty \\
\infty\end{array}\right|$ & $\begin{array}{l}r \\
\dot{0} \\
+\end{array}$ & $\left|\begin{array}{l}\infty \\
\dot{0} \\
m\end{array}\right|$ & $\stackrel{n}{0}$ & \begin{tabular}{l|l}
0 & $\alpha$ \\
$\dot{0}$ & \\
&
\end{tabular} & 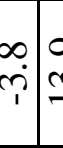 & $\vec{r}$ & \\
\hline $\begin{array}{l}\underset{\hat{\sigma}}{\hat{\sigma}} \\
\text { 艿 }\end{array}$ & $\stackrel{\sim}{\grave{N}}$ & $\begin{array}{l}\nabla \\
\stackrel{\leftrightarrow}{0}\end{array}$ & $\stackrel{r}{\tilde{\sigma}}$ & ri & $\begin{array}{l}0 \\
\dot{0} \\
\stackrel{1}{1}\end{array}$ & $\left|\begin{array}{l}\infty \\
i \\
2\end{array}\right|$ & $\stackrel{n}{\ddot{n}}$ & $\begin{array}{l}+ \\
\dot{0} \\
\sim\end{array}$ & $\begin{array}{l}? \\
\pm \\
\Xi\end{array}$ & $\because$ & 官 & $\left|\begin{array}{l}n \\
n \\
m\end{array}\right|$ & శ̊? & \begin{tabular}{l|r}
$\infty$ & $r$ \\
$\grave{N}$ & $r$
\end{tabular} & 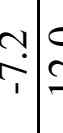 & $\begin{array}{l}\vec{i} \\
\mathrm{v}\end{array}$ & \\
\hline ळి & 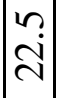 & $\Xi$ & $\begin{array}{l}0 \\
\dot{j} \\
\mathcal{f}\end{array}$ & $\frac{N}{m}$ & 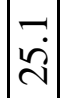 & 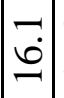 & $\mid \begin{array}{l}\infty \\
\dot{\gamma} \\
\gamma\end{array}$ & $\begin{array}{l}+ \\
\dot{\sim} \\
\dot{N}\end{array}$ & $\mid \begin{array}{l}0 \\
\dot{0} \\
0\end{array}$ & $\dot{\vec{r}}$ & $\frac{r}{\dot{\gamma}}$ & $\begin{array}{c}\dot{v} \\
\dot{m} \\
m\end{array}$ & $\begin{array}{ll}0 \\
\vec{b}\end{array}$ & $\begin{array}{c}n \\
\\
\end{array}$ & 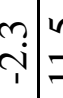 & $?$ & $\frac{n}{2}$ \\
\hline$\frac{9}{\hat{a}}$ & $\begin{array}{l}n \\
\sim \\
\sim\end{array}$ & $\begin{array}{l}a \\
\text { in }\end{array}$ & $\underset{\dot{\gamma}}{\rightleftarrows}$ & 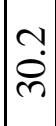 & $\mid \begin{array}{l}\forall \\
\dot{0} \\
\stackrel{v}{*}\end{array}$ & $\begin{array}{c}0 \\
\infty \\
-\end{array}$ & $\mid \begin{array}{l}7 \\
\dot{0} \\
+\end{array}$ & $\stackrel{n}{\sim}$ & $\mid \begin{array}{c}0 \\
\dot{0} \\
.\end{array}$ & $\vec{\forall}$ & $\begin{array}{l}\infty \\
\infty \\
\tilde{m}\end{array}$ & $\frac{a}{m}$ & 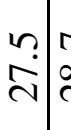 & 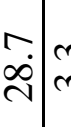 & $m=$ & \pm & 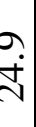 \\
\hline 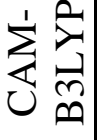 & $\mid \begin{array}{l}\infty \\
\dot{\lambda} \\
\sim\end{array}$ & a & $\begin{array}{l}\infty \\
\dot{\gamma} \\
\forall\end{array}$ & 苗 & $\stackrel{-}{\check{n}}$ & $\left|\begin{array}{c}0 \\
i \\
2\end{array}\right|$ & $\mid \begin{array}{c}\Upsilon \\
\infty \\
+ \\
+\end{array}$ & 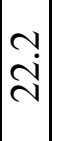 & $\stackrel{0}{0}$ & $\begin{array}{l}\infty \\
\dot{m}\end{array}$ & $\begin{array}{l}\infty \\
\dot{\mathcal{f}} \\
\mathcal{f}\end{array}$ & $\mid \begin{array}{c}0 \\
\dot{+} \\
m\end{array}$ & 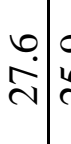 & 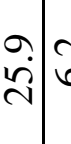 & 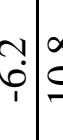 & 足 & $\vec{\partial}$ \\
\hline$\frac{\partial}{\not}$ & $\underset{\infty}{\infty}$ & $n$ & $\left|\begin{array}{c}m \\
\infty \\
m\end{array}\right|$ & $\begin{array}{l}\infty \\
\\
\end{array}$ & 足 & 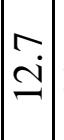 & $\begin{array}{l}\exists \\
\end{array}$ & $\begin{array}{c}N \\
0 \\
-\end{array}$ & n? & $\mid \begin{array}{l}0 \\
\\
1\end{array}$ & $\frac{n}{m}$ & $\begin{array}{c}0 \\
\dot{0} \\
m\end{array}$ & $\begin{array}{l}n \\
\tilde{n} \\
z\end{array}$ & 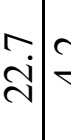 & \begin{tabular}{l|l}
$r$ \\
$\stackrel{r}{r}$
\end{tabular} & $\infty$ & ב. \\
\hline $\bar{a}^{a}$ & 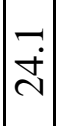 & $\mid \begin{array}{l}0 \\
0 \\
-1\end{array}$ & $\begin{array}{l}0 \\
\dot{f}\end{array}$ & $\begin{array}{l}m \\
n \\
m\end{array}$ & $\overrightarrow{\dot{\rho}}$ & $\left|\begin{array}{l}0 \\
\dot{\sim}\end{array}\right|$ & $\begin{array}{c}-\dot{n} \\
\dot{n}\end{array}$ & $\frac{a}{\lambda}$ & $\begin{array}{l}n \\
\ddots \\
\ddots\end{array}$ & $\mid \begin{array}{c}\infty \\
0 \\
0\end{array}$ & $\mid \begin{array}{c}0 \\
\dot{\gamma} \\
\dot{\gamma}\end{array}$ & $\begin{array}{l}m \\
m \\
m\end{array}$ & $\begin{array}{l}0 \\
\dot{m}\end{array}$ & $\begin{array}{l}\stackrel{+}{\infty} \\
\stackrel{\infty}{v}\end{array}$ & $\begin{array}{c}-1 \\
6\end{array}$ & D. & $\frac{0}{\mathrm{v}}$ \\
\hline$\frac{\tilde{n}}{\infty}$ & $\begin{array}{l}\infty \\
\sim \\
\sim\end{array}$ & $\begin{array}{l}0 \\
i \\
-1\end{array}$ & $\begin{array}{l}\mathcal{Z} \\
\dot{f}\end{array}$ & $\begin{array}{l}a \\
\dot{m}\end{array}$ & ふे. & $\stackrel{n}{\stackrel{\sim}{\sim}}$ & $\mid \begin{array}{c}0 \\
\tilde{n} \\
n\end{array}$ & $\frac{n}{\sim}$ & $\underset{\dot{\Xi}}{\Xi}$ & m & 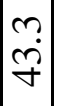 & $\begin{array}{l}0 \\
\bar{m} \\
m\end{array}$ & ¿̇. & 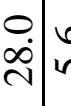 & $\begin{array}{l}6 \\
\dot{r} \\
1\end{array}$ & $=$ & $m$ \\
\hline $\begin{array}{l}0 \\
\infty \\
\infty \\
\infty\end{array}$ & $\frac{\sim}{\sim}$ & $\stackrel{\sim}{\sim}$ & $\underset{\dot{\gamma}}{-}$ & $\begin{array}{l}\dot{\nabla} \\
\stackrel{\sim}{*}\end{array}$ & 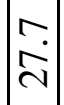 & $\stackrel{\sim}{\mathrm{N}}$ & $\stackrel{\nabla}{\circ}$ & $\begin{array}{l}a \\
\infty \\
-1\end{array}$ & $\begin{array}{l}0 \\
\stackrel{1}{1}\end{array}$ & $m$ & $\begin{array}{r}\dot{P} \\
\dot{q}\end{array}$ & ru & $\begin{array}{c}\text { Na } \\
\infty \\
\sim \\
c\end{array}$ & 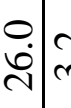 & $\begin{array}{l}c \\
m \\
m\end{array}$ & $\dot{J}$ & $\dot{\square}$ \\
\hline 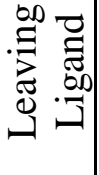 & $\frac{\pi}{2}$ & $\frac{\pi}{2}$ & $\frac{\infty}{2}$ & $\begin{array}{l}-1 \\
0 \\
0 \\
0\end{array}$ & 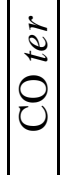 & 0 & O & $\frac{m}{\infty}$ & $\frac{m}{2}$ & $\frac{m}{2}$ & $\frac{m}{2}$ & $\begin{array}{c}0 \\
\vdots \\
0 \\
0\end{array}$ & $\begin{array}{c}\bar{\Xi} \\
0 \\
0\end{array}$ & 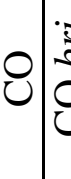 & 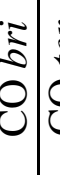 & $\begin{array}{l}5 \\
0 \\
0\end{array}$ & 0 \\
\hline 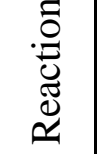 & -1 & $\sim \mid$ & $m$ & $\nabla$ & $n$ & 6 & 1 & $\infty$ & $a$ & 의 & 二 & $\stackrel{\sim}{-1}$ & $\cong$ & $\pm v$ & $\therefore=$ & 0 & - \\
\hline
\end{tabular}


reduces its ability to accept electrons from the other Ir atom. Such asymmetry in Ir-C (bri) bond lengths was observed in tetrairidium clusters and can be considered as a transformation from a bridging carbonyl to terminal one binding to the Ir atom with high electron density.[87,88] The changes in the LDEs are similar to the trends predicted for $\operatorname{Ir}\left(\mathrm{PH}_{3}\right)_{y}(\mathrm{CO})_{z}$. As more $\mathrm{PH}_{3}$ 's are substituted, the LDEs of the carbonyl increase moderately, whereas those of the $\mathrm{PH}_{3}$ 's decrease slightly, and both the Ir-C and Ir-P bond lengths decrease. For coordinatively saturated $\operatorname{Ir}_{2}\left(\mathrm{PH}_{3}\right)_{\mathrm{y}}(\mathrm{CO})_{\mathrm{z}}$ clusters, most LDEs range from $\sim 15$ to $\sim 35 \mathrm{kcal} / \mathrm{mol}$, closer to those of $\mathrm{IrL}_{4}$ and less than for $\mathrm{IrL}_{3}$.

Table 6. Calculated NBO Charges on $\operatorname{Ir}^{a}{ }^{a}$ Average C-O Stretching Frequencies $\left(v_{\mathrm{C}-O}\right),{ }^{a}$ Ir-Ir Bond Energies (BDE), ${ }^{\mathrm{b}}$ Bond Distances $(r)^{\mathrm{a}}$, and LDEs for $\operatorname{Ir}_{2}\left(\mathrm{PH}_{3}\right)_{\mathrm{y}}(\mathrm{CO})_{\mathrm{z}}{ }^{\mathrm{b}}$

\begin{tabular}{|l|c|c|c|}
\hline Property & $\mathrm{Ir}_{2}(\mathrm{CO})_{8} \mathrm{C}_{2 \mathrm{v}}$ & $\mathrm{Ir}_{2} \mathrm{PH}_{3}(\mathrm{CO})_{7}(\mathbf{2 d})$ & $\mathrm{Ir}_{2}\left(\mathrm{PH}_{3}\right)_{2}(\mathrm{CO})_{6}(\mathbf{2 l})$ \\
\hline Ir NBO charge & 0.22 & $0.23^{\mathrm{c}}, 0.06^{\mathrm{d}}$ & 0.07 \\
\hline Ir NBO 5d & 8.21 & $8.20^{\mathrm{c}}, 8.33^{\mathrm{d}}$ & 8.32 \\
\hline$r(\mathrm{Ir}-\mathrm{Ir})(\AA)$ & 2.774 & 2.760 & 2.744 \\
\hline BDE $(\mathrm{Ir}-\mathrm{Ir})(\mathrm{kcal} / \mathrm{mol})$ & 45.7 & 52.7 & 57.2 \\
\hline Average $v_{\mathrm{C}-\mathrm{O}}\left(\mathrm{cm}^{-1}\right)$ & 2026 & 2002 & 1972 \\
\hline$r(\mathrm{C}-\mathrm{O})($ ter,$\AA)$ & 1.152 & 1.154 & 1.156 \\
\hline$r(\mathrm{C}-\mathrm{O})($ bri,$\AA)$ & 1.180 & 1.186 & 1.193 \\
\hline$r(\operatorname{Ir}-\mathrm{C})^{\mathrm{c}}($ ter,$\AA)$ & 1.927 & $1.925^{\mathrm{c}}, 1.916^{\mathrm{d}}$ & 1.914 \\
\hline$r(\operatorname{Ir}-\mathrm{C})^{\mathrm{c}}($ bri,$\AA)$ & 2.090 & $2.106^{\mathrm{c}}, 2.061^{\mathrm{d}}$ & 2.075 \\
\hline $\mathrm{LDE}($ ter $\mathrm{CO})(\mathrm{kcal} / \mathrm{mol})$ & & 25.8 & 28.3 \\
\hline $\mathrm{LDE}($ bri $\mathrm{CO})(\mathrm{kcal} / \mathrm{mol})$ & & 30.8 & 33.2 \\
\hline$r(\operatorname{Ir}-\mathrm{P})(\AA)$ & & 2.309 & 2.300 \\
\hline $\mathrm{LDE}\left(\mathrm{PH}{ }_{3}\right)(\mathrm{kcal} / \mathrm{mol})$ & & 28.6 & 27.4 \\
\hline
\end{tabular}

${ }^{\text {a }}$ All bond distances, frequencies, and NBO charges are calculated at the SVWN5 level.

${ }^{\mathrm{b}}$ All BDEs and LDEs are calculated by CCSD(T) at the SVWN5 optimized geometries.

${ }^{\mathrm{c}}$ Ir not bonded to $\mathrm{PH}_{3}$.

${ }^{\mathrm{d}}$ Ir bonded to $\mathrm{PH}_{3}$.

\subsection{DFT Benchmarks for the LDEs for Ir and $\operatorname{Ir}_{2}$ Clusters.}

The catalytic character of tetrairidium clusters is generally associated with the exposed metal core that is generated by the dissociation of ligands.[89] We have discussed the LDEs of $\operatorname{Ir}\left(\mathrm{PH}_{3}\right)_{y}(\mathrm{CO})_{\mathrm{z}}$ and $\operatorname{Ir}_{2}\left(\mathrm{PH}_{3}\right)_{\mathrm{y}}(\mathrm{CO})_{\mathrm{z}}$ clusters at the CCSD(T) level. It is difficult to perform $\operatorname{CCSD}(\mathrm{T})$ calculations for the ligated tetrairidium clusters because of their size, and so we need to find a DFT functional that can be used to predict the LDEs of the larger clusters. The functionals used for the benchmarking and their LDEs are given in Tables 2 and 5. Generalized gradient approximation (GGA) hybrid functionals with long-range corrections (LR-HGGA), CAM-B3LYP, $\omega B 97 X$, and $\omega B$ 97X-D perform the best, followed by hybrid GGA functionals (M06, B3LYP, B98) and pure GGA functionals (BP86, PBE, and PW91). Although SVWN5, 
a local density approximation (LDA) functional, gives good predictions of the geometry, the LDEs are predicted to be too large, as expected for the overbinding of local functionals. The energies of $\operatorname{Ir}_{4}\left(\mathrm{PH}_{3}\right)_{\mathrm{y}}(\mathrm{CO})_{\mathrm{z}}$ clusters were thus calculated at the $\omega \mathrm{B} 97 \mathrm{X}-\mathrm{D}$ level, which has deviations of less than $5 \mathrm{kcal} / \mathrm{mol}$ from CCSD(T) results for $\operatorname{Ir}\left(\mathrm{PH}_{3}\right)_{\mathrm{y}}(\mathrm{CO})_{\mathrm{z}}$ and $\operatorname{Ir}_{2}\left(\mathrm{PH}_{3}\right)_{\mathrm{y}}(\mathrm{CO})_{\mathrm{z}}$ clusters.

\section{4 $\operatorname{Ir}_{4}\left(\mathrm{PH}_{3}\right)_{\mathrm{y}}(\mathrm{CO})_{\mathrm{z}}$ structures, relative energies, and dissociation energies.}

Because it has been shown experimentally [40] that no more than four carbonyls can be substituted by phosphines, only the $\operatorname{Ir}_{4} \mathrm{PH}_{3}(\mathrm{CO})_{11}, \operatorname{Ir}_{4}\left(\mathrm{PH}_{3}\right)_{2}(\mathrm{CO})_{10}$, $\mathrm{Ir}_{4}\left(\mathrm{PH}_{3}\right)_{3}(\mathrm{CO})_{9}$, and $\mathrm{Ir}_{4}\left(\mathrm{PH}_{3}\right)_{4}(\mathrm{CO})_{8}$ clusters are considered here. Besides the lowest energy isomers characterized by experiment, other isomers with substitution at various positions were also investigated, including both $\mathrm{C}_{3 \mathrm{v}}$ and $\mathrm{T}_{\mathrm{d}}$ structures and terminal and bridging position substitution and dissociation. In total, $87 \operatorname{Ir}_{4}\left(\mathrm{PH}_{3}\right)_{\mathrm{y}}(\mathrm{CO})_{\mathrm{z}}$ isomers have been optimized, as shown in Figure 4; their bond distances are given in the SI, and relative energies are listed in Tables 7 and 8 .

Different DFT functionals give different relative energies for the $\operatorname{Ir}_{4}(\mathrm{CO})_{12}$ cluster. $\omega \mathrm{B} 97 \mathrm{X}-\mathrm{D}$, which we use to predict the energetics of the $\operatorname{Ir}_{4}\left(\mathrm{PH}_{3}\right)_{\mathrm{y}}(\mathrm{CO})_{\mathrm{z}}$ isomers, correctly predicts that the lowest energy isomer of $\operatorname{Ir}_{4}(\mathrm{CO})_{12}$ is the $\mathrm{T}_{\mathrm{d}}$ isomer.[51] The axial sites in the $\mathrm{C}_{3 \mathrm{v}}$ structures are less crowded, and axial site substitution by $\mathrm{PH}_{3}$ is favoured and controlled by steric effects. Whether a $\mathrm{C}_{3 \mathrm{v}}$ or $\mathrm{T}_{\mathrm{d}}$ structure is adopted is determined by the $\pi$-acidity of the substituting ligands (i.e., the exact structure is controlled by electronic effects).[38,39,40] As the electronegativity of the groups bonded to phosphorus increases, the contribution of the phosphorus to the LUMO that accepts $\pi$-electrons from Ir atom increases. Thus, the order of the $\pi$-acidity for common phosphine ligands is $\mathrm{PR}_{3}<\mathrm{PAr}_{3}<\mathrm{P}(\mathrm{OR})_{3}<\mathrm{P}(\mathrm{OAr})_{3}<\mathrm{PF}_{3}(\mathrm{R}=$ alkyl).[90] It has been reported that a good $\pi$-acceptor phosphite ligand, $\mathrm{P}\left(\mathrm{OCH}_{2}\right)_{3} \mathrm{CEt}$, can maintain the $T_{d}$ structure in monosubstitution of $\operatorname{Ir}_{4}(\mathrm{CO})_{12}$, [39] whereas for some poor $\pi$-acceptors, such as $\mathrm{PPh}_{2} \mathrm{Me}$, substitution leads to a $\mathrm{C}_{3 \mathrm{v}}$ structure.[39,91] The $\mathrm{PH}_{3}$ ligand used in the current investigation is a stronger $\pi$-acceptor than most phosphines but is not as strong as phosphites. Most of these phosphines and phosphites are poor $\pi$-acceptors as compared with carbonyl. Our calculations show that the $T_{d}$ structure of $\mathrm{Ir}_{4} \mathrm{PH}_{3}(\mathrm{CO})_{11}$ is lower in energy by only $0.3 \mathrm{kcal} / \mathrm{mol}$ than the $\mathrm{C}_{3 \mathrm{v}}$ structure. As more $\mathrm{CO}$ ligands are replaced by $\mathrm{PH}_{3}$ ligands, the $\mathrm{C}_{3 \mathrm{v}}$ structure becomes more favoured.

The NBO charges on Ir atoms were calculated to provide more insight into the chemistry. We discuss changes in the Ir NBO charge relative to that in $\mathrm{T}_{\mathrm{d}} \operatorname{Ir}_{4}(\mathrm{CO})_{12}$ as given in Equation (2):

$$
\Delta \mathrm{NBO}=\left[\mathrm{NBO}_{\mathrm{Ir} 4(\mathrm{CO}) 12(\mathrm{Td})} \text { charge }\right]-\left[\text { average } \mathrm{NBO}_{\mathrm{Ir} 4(\mathrm{PH} 3) \mathrm{y}(\mathrm{CO}) \mathrm{z}} \text { charge }\right]
$$

These $\triangle \mathrm{NBO}$ values with their relative energies are given in Table 8 . For $\mathrm{Ir}_{4} \mathrm{PH}_{3}(\mathrm{CO})_{11}$ isomers (4a, 4b, 4d, and 4e), although one $\mathrm{PH}_{3}$ ligand has been introduced, the Ir NBO charge does not change much (-0.01 to 0.02$)$, and so the $\mathrm{T}_{\mathrm{d}}$ structure is maintained. From $\mathrm{Ir}_{4}\left(\mathrm{PH}_{3}\right)_{2}(\mathrm{CO})_{10}$ to $\mathrm{Ir}_{4}\left(\mathrm{PH}_{3}\right)_{4}(\mathrm{CO})_{8}$ isomers $(6 \mathbf{a}, 6 \mathbf{c}, \mathbf{6 d}, 6 \mathbf{e}, 8 \mathbf{a}, 8 \mathbf{b}, 8 \mathbf{d}, 8 \mathbf{e}, 10 \mathbf{a}$, 10b, and 10d), the negative charge on the Ir atom and $\triangle \mathrm{NBO}$ increases, and our calculations predict that the $\mathrm{C}_{3 \mathrm{v}}$ isomers are more stable than the $\mathrm{T}_{\mathrm{d}}$ isomers. These trends suggest that the average charge on the Ir atom in tetra-iridium clusters plays a role in the energetics of the clusters. When a carbonyl is replaced by a ligand that has a similar $\pi$-acidity, the $T_{d}$ structure is maintained, because the Ir charge does not change 
much. If it is a weak $\pi$-acceptor that increases the electron density on the Ir atom, the $\mathrm{C}_{3 \mathrm{v}}$ structure is adopted, because in this structure, an $\mathrm{Ir}$ atom can bind to more carbonyls and transfer more electron density to get closer to the charge in $\operatorname{T}_{d} \operatorname{Ir}_{4}(C O)_{12}$.

There is an apparent equilibrium between increased $\pi$-back-bonding and lower electron repulsion in the $\sigma$ orbitals. Steric effects are lower in the $\mathrm{C}_{3 \mathrm{v}}$ structure, leading to an additional decrease in energy. The energy differences between the $T_{d}$ and $C_{3 v}$ structures are often not large, and thus, an interconversion between isomers with $a x$ ligands and $e q$ ligands is often observed experimentally. This was explained by Cotton in terms of a merry-go-round mechanism.[92,93] Different from the isomers discussed above that have terminal $\mathrm{PH}_{3}$ ligands, those with bridging $\mathrm{PH}_{3}$ ligands $(\mathbf{4 c}, \mathbf{6 b}, \mathbf{8 c}$, and 10c) have higher energies than other isomers by 7 to $10 \mathrm{kcal} / \mathrm{mol}$. This difference results because the phosphine lone pair electrons have high s character and cannot bind efficiently to two Ir atoms at the same time.

Carbonyl dissociation from a coordinatively saturated tetrairidium cluster can lead to a hydrogen atom transfer from the $\mathrm{PH}_{3}$ to a nearby $\mathrm{Ir}$ atom, analogous to reaction (15) for $\operatorname{Ir}_{2}\left(\mathrm{PH}_{3}\right)_{\mathrm{y}}(\mathrm{CO})_{\mathrm{z}}$. If one bridging carbonyl is dissociated from a tetrairidium cluster that has a bridging $\mathrm{PH}_{3}$, a $\mathrm{H}$ atom transfer always takes place, as shown in Figure 5 (a) (this corresponds to reactions (14), (34), (60), and (79) in Figure 4). As a bridging carbonyl is removed, an eq $\mathrm{CO}$ moves to the bri position of the removed $\mathrm{CO}$, and an $\mathrm{H}$ atom transfers to the original position of the eq $\mathrm{CO}$ and binds to the $\mathrm{Ir}$ atom to maintain Ir coordination. In the final product, there is a bridging phosphido group. Comparing $\mathbf{4 c}$ with $\mathbf{5 k}$, we see that the total reaction can be regarded as a dissociation of eq $\mathrm{CO}$, compensated by a hydrogen atom from a $\mathrm{PH}_{3}$ and keeping the cluster coordinatively saturated. A second type of hydrogen atom transfer is illustrated in Figure 5 (b) (which corresponds to reaction 70 in Figure 4). Dissociation of a bridging carbonyl leads to rotation of an adjacent $\mathrm{PH}_{3}$ to form a bridge $\mathrm{PH}_{2}$ group and a hydrogen bonded to Ir to maintain as high a coordination at Ir as possible. A similar reaction has been reported in which a basal phosphine monosubstituted tetrairidium cluster yields a product with both a bridging phosphido and a bridging $\mathrm{H}$ atom by deprotonation of the phosphine.[94] However, in our investigation, no $\mathrm{H}$ atom transfers involving basal $\mathrm{PH}_{3}$ have been found for $\mathrm{C}_{3 \mathrm{v}}$ isomers. The two kinds of $\mathrm{H}$ atom transfers described above correspond (a) to transfer from a bridging $\mathrm{PH}_{3}$ that does not bind efficiently because of its lone pair orientation and (b) to transfer from an apical (api = apical) $\mathrm{PH}_{3}$ that has the lowest dissociation energy (see below). These results suggest that each $\mathrm{PH}_{3}$ with a low LDE is more likely to lose an $\mathrm{H}$ atom forming a lower energy structure with a bridging phosphido group.

For all $\mathrm{CO}$ and $\mathrm{PH}_{3}$ dissociation processes, there are no transformations from $\mathrm{C}_{3 \mathrm{v}}$ structures to $\mathrm{T}_{\mathrm{d}}$ structures, but the reverse is possible. The third type of $\mathrm{H}$ atom transfer (Figure 5 (c)) is an example of such a transformation (reactions (65) and (82) in Figure 4). In a $\mathrm{T}_{\mathrm{d}}$ isomer, a terminal $\mathrm{PH}_{3}$ moves close to an unsaturated $\mathrm{Ir}$ atom as a result of a carbonyl dissociation, and one of its $\mathrm{H}$ atoms moves farther to bind to the Ir atom to maintain coordination, leaving a bridging phosphido group. During this process, two carbonyls are converted into bridging carbonyls, and the isomer switches to a $\mathrm{C}_{3 \mathrm{v}}$ structure. 


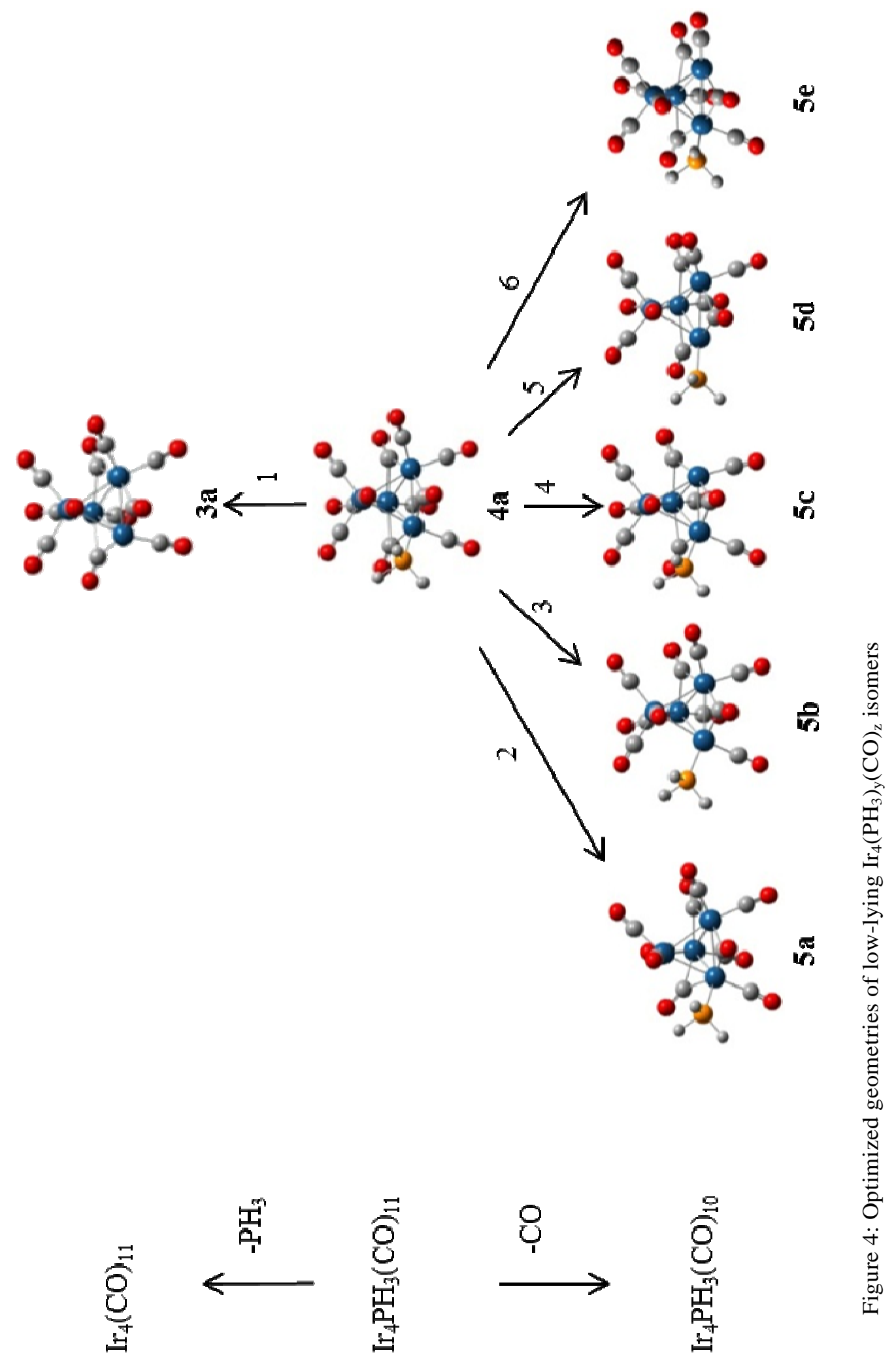




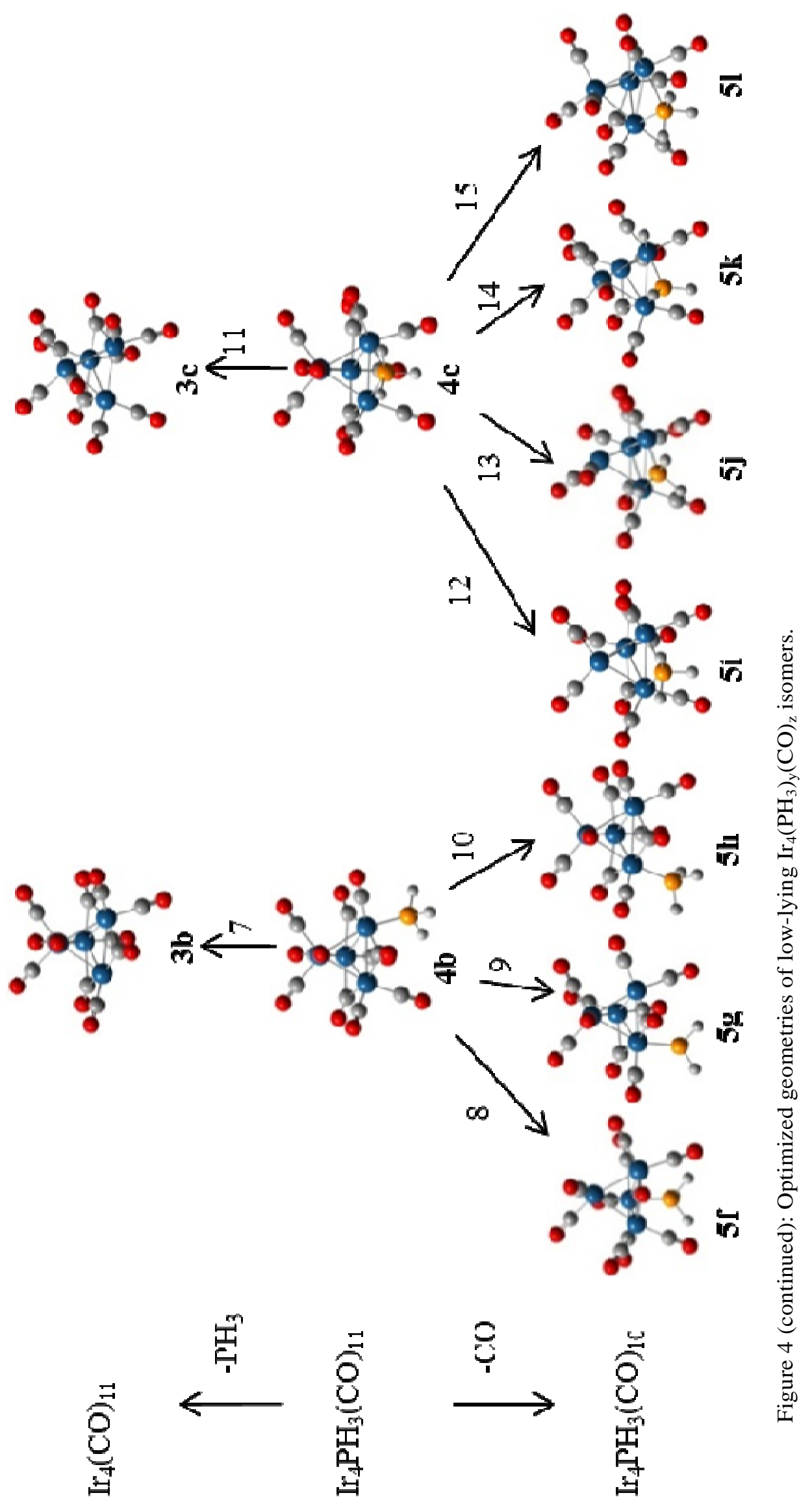




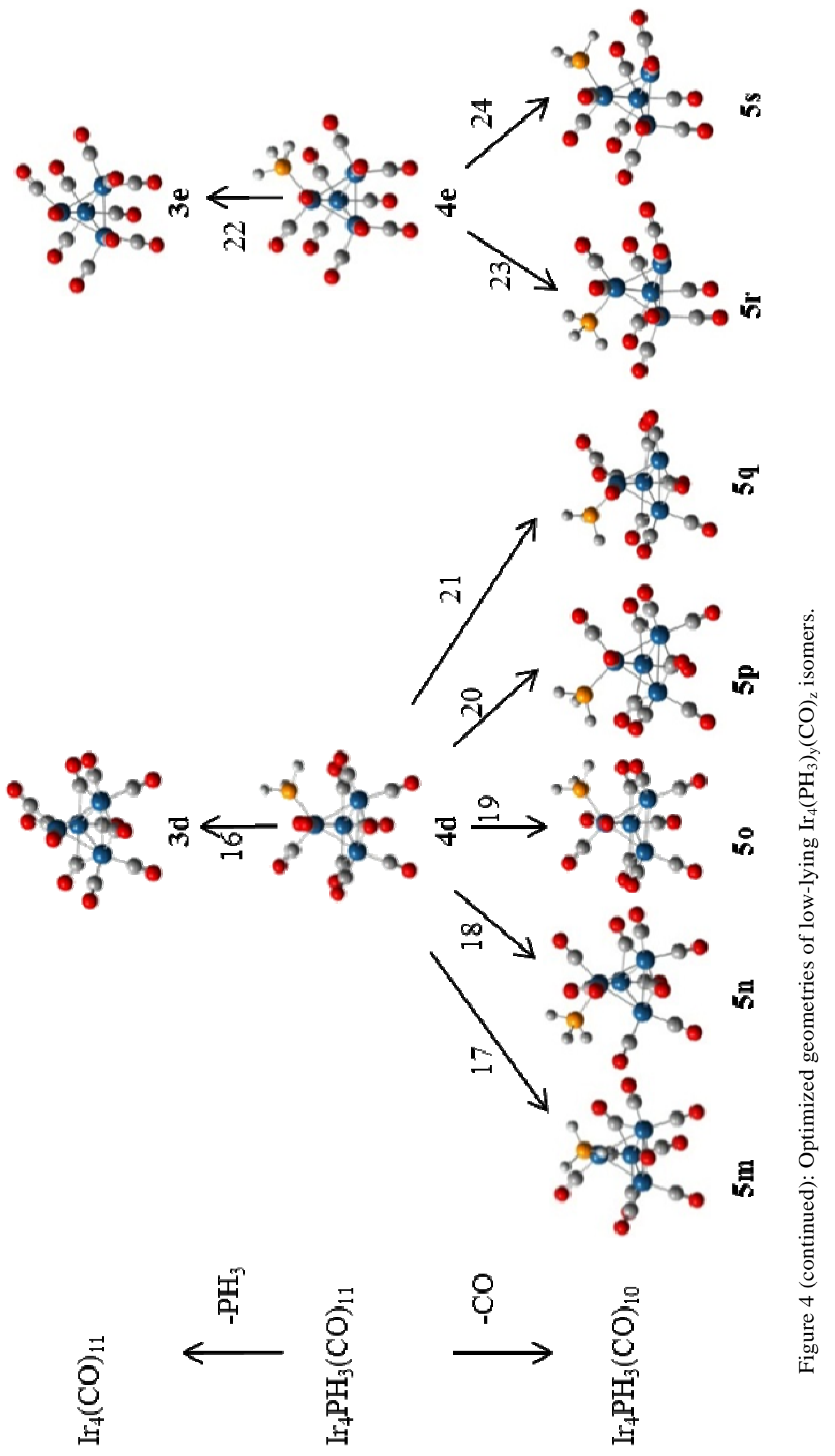




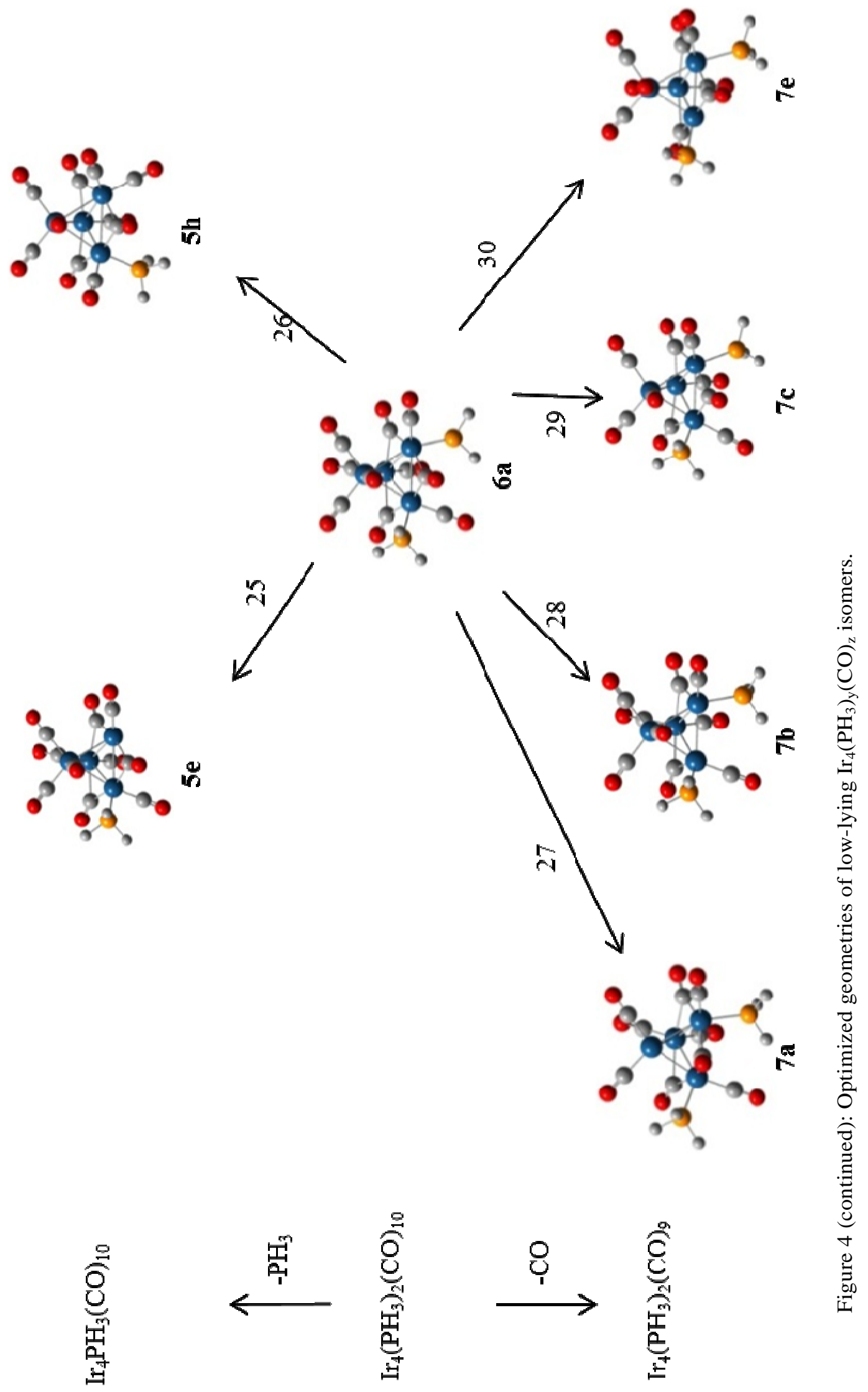




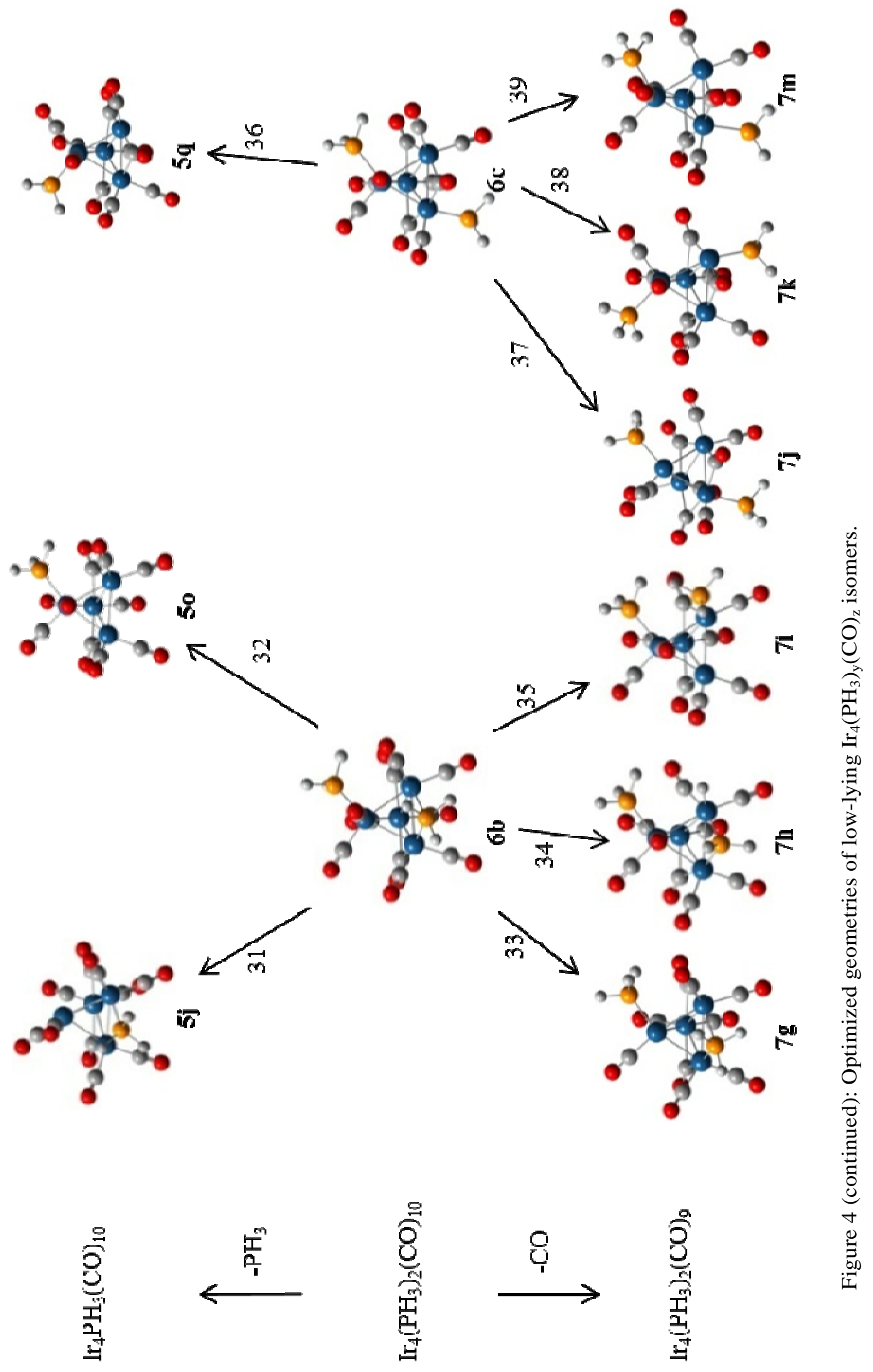



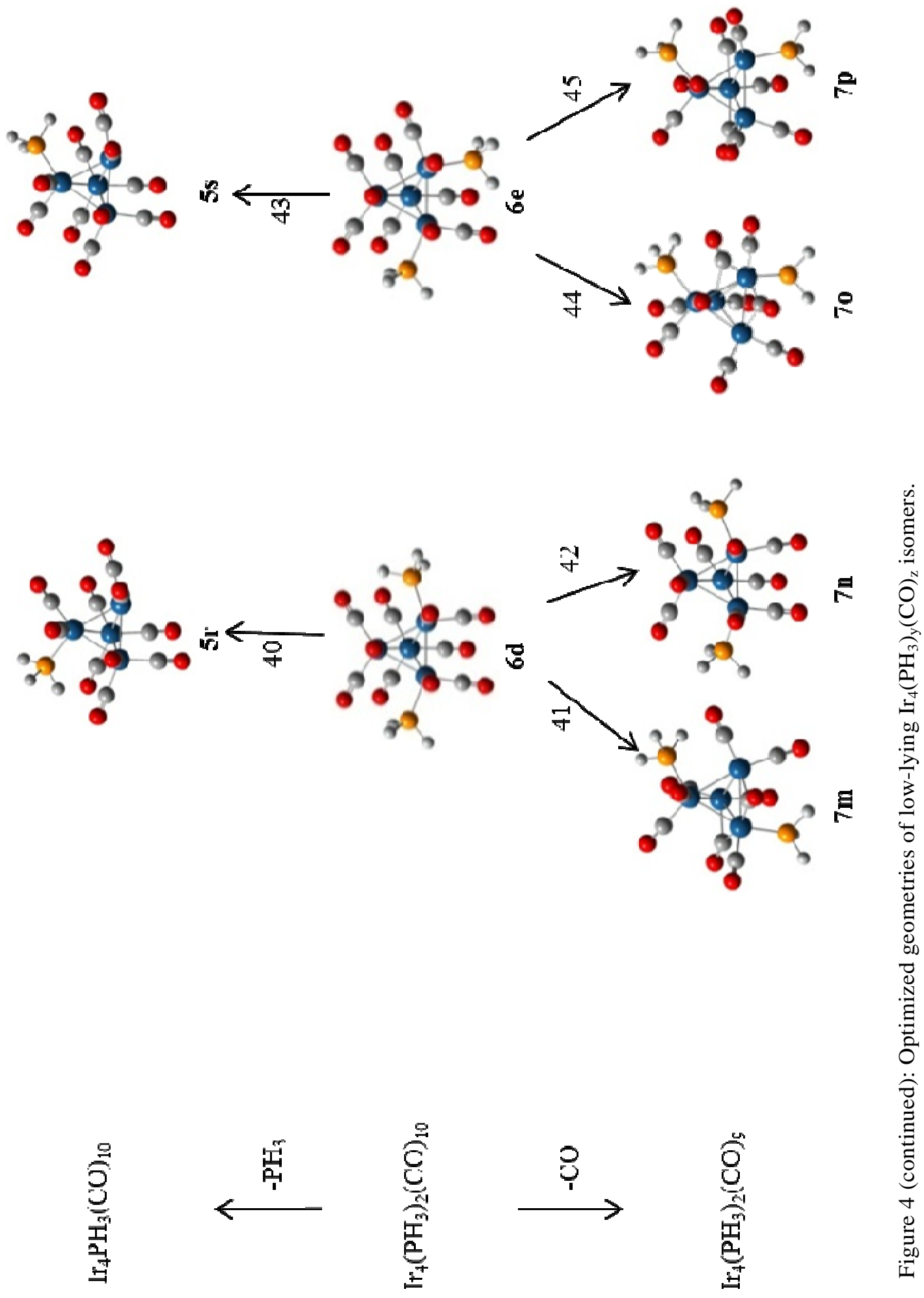


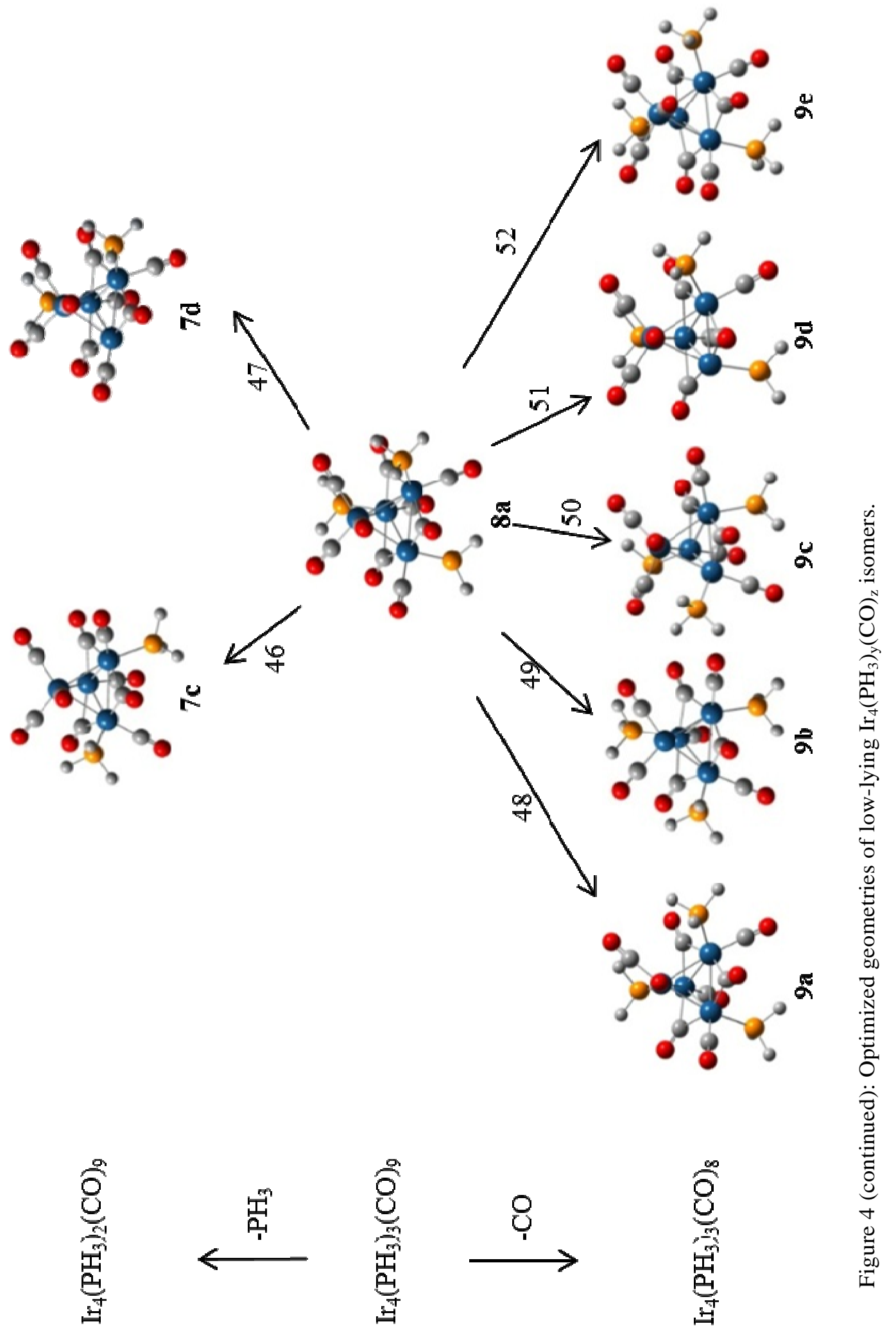



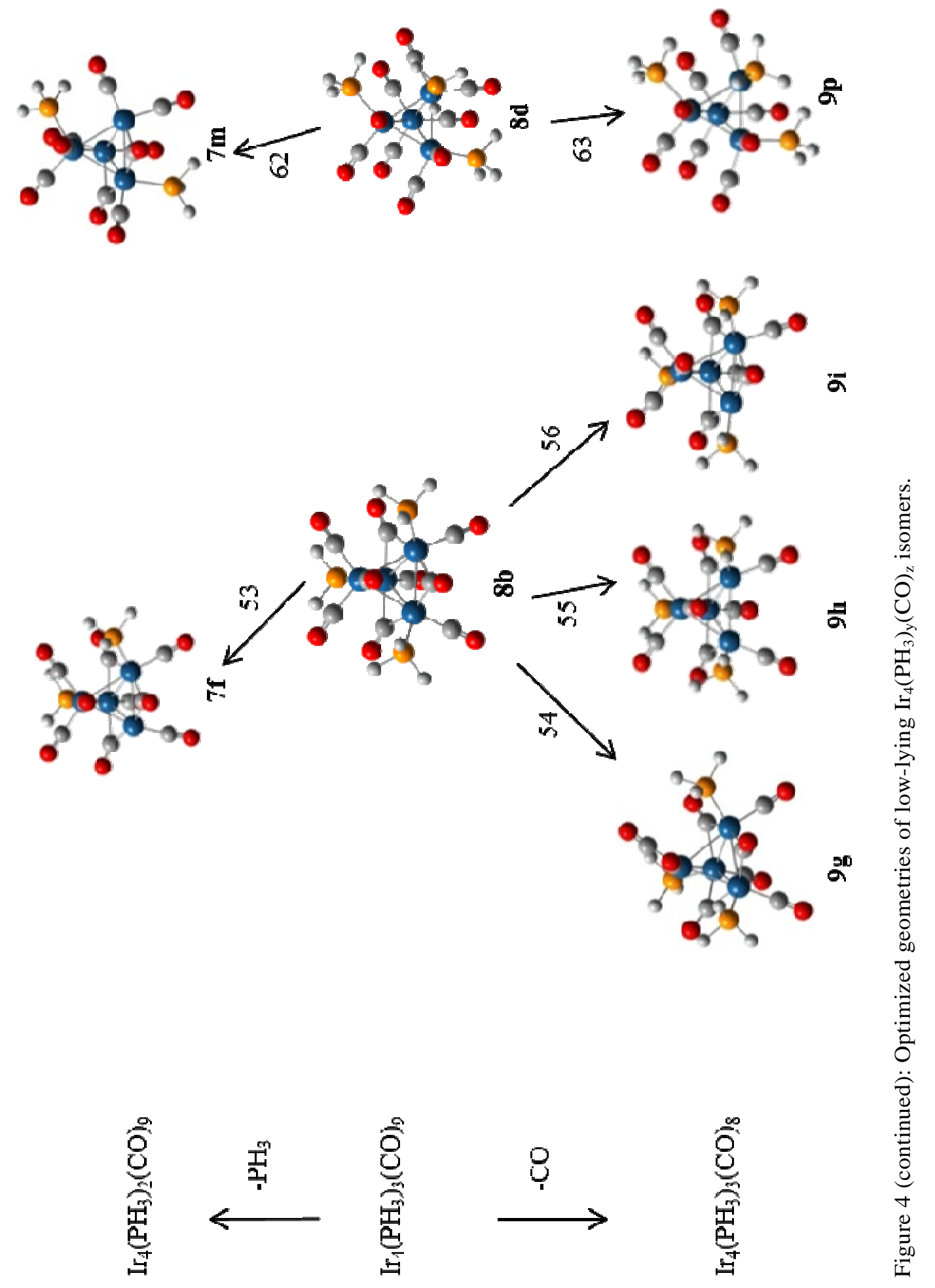


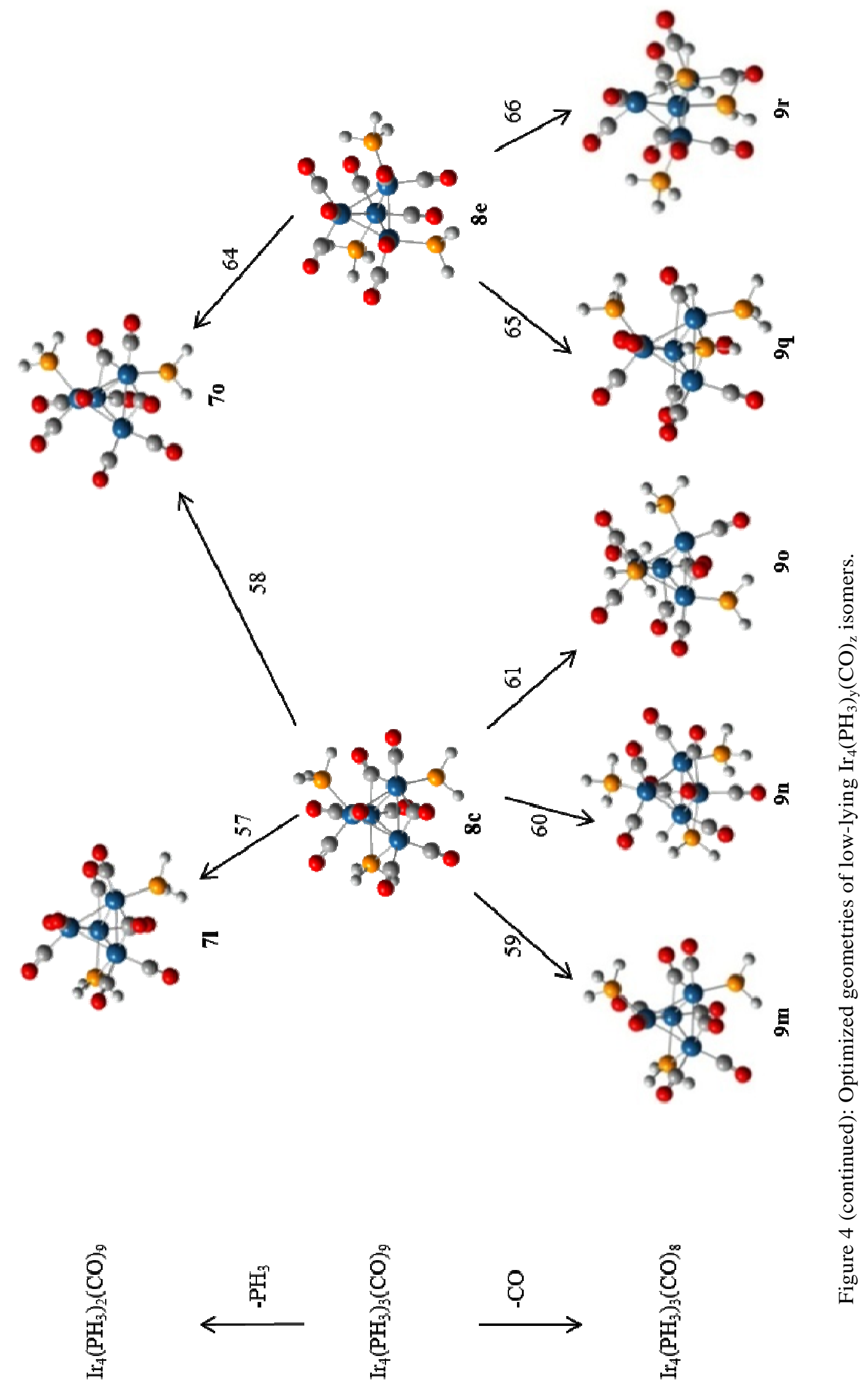




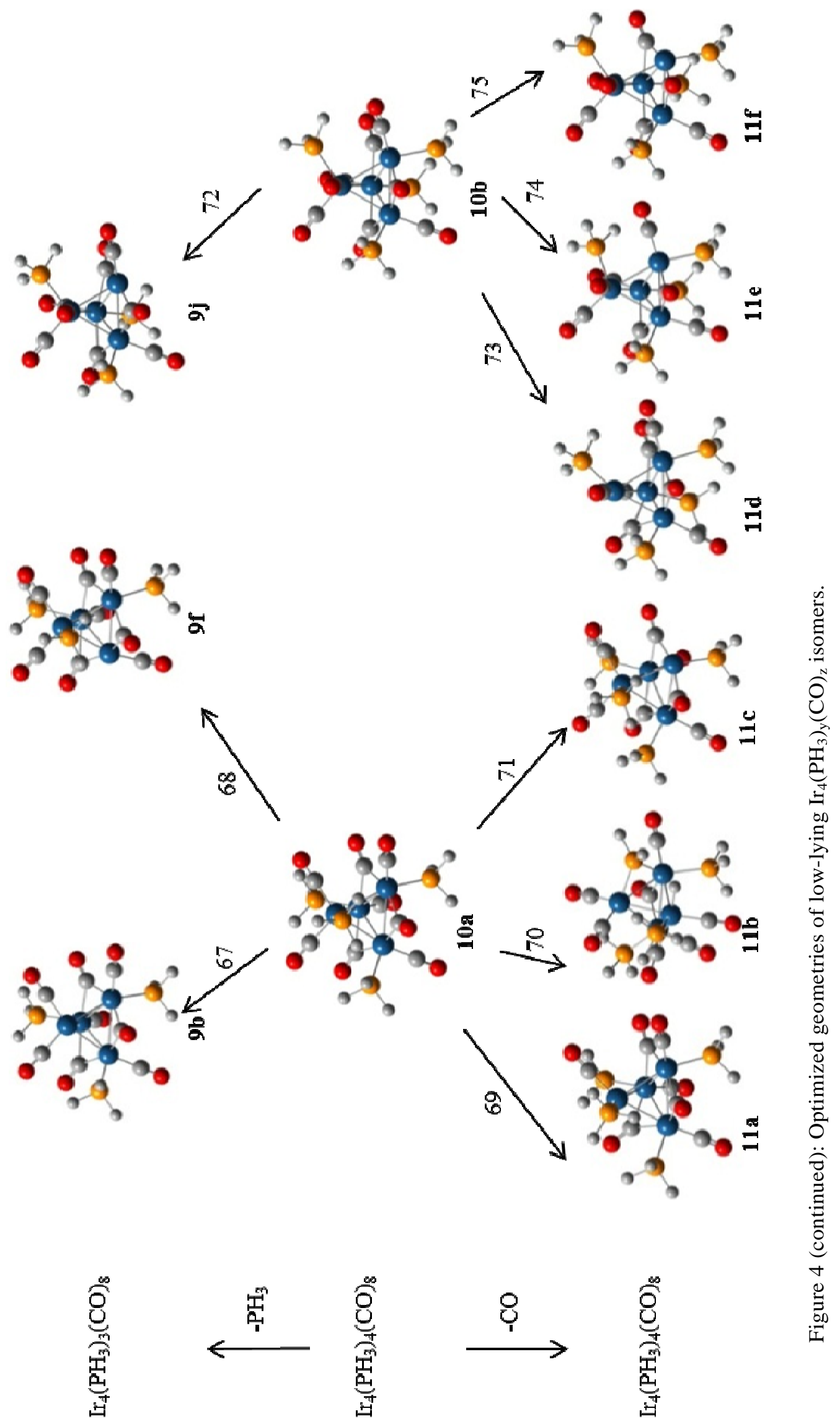



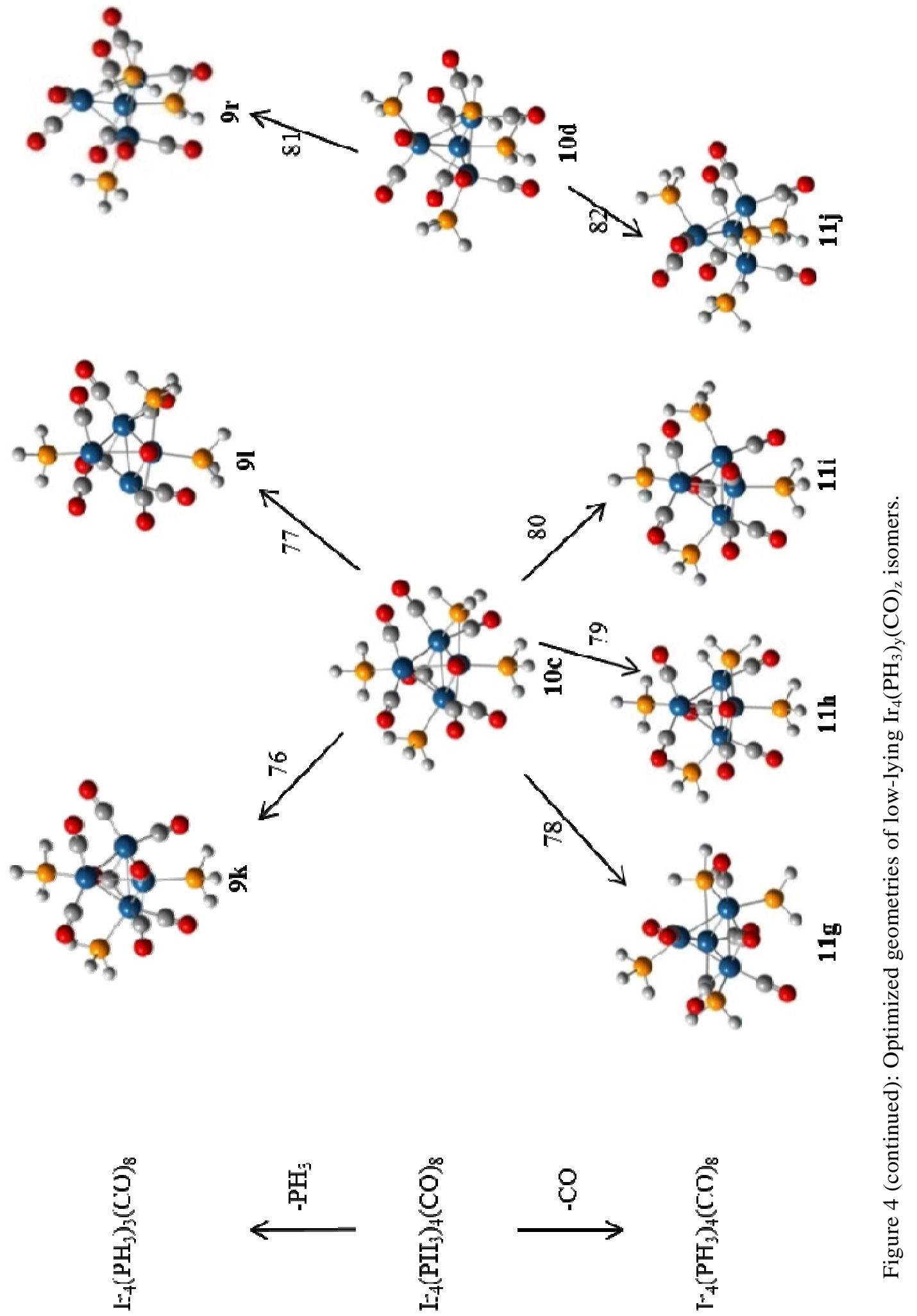


\begin{tabular}{|c|c|c|c|c|c|c|c|c|c|}
\hline & & in & 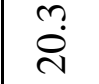 & & & & & & \\
\hline & & in & $\vec{d}$ & & & $\check{a}$ & $\stackrel{m}{\sim}$ & & \\
\hline & & - & $\stackrel{n}{n}$ & & & శ్ & $\stackrel{0}{0}$ & & \\
\hline & & in & बें & $\approx$ & $\begin{array}{l}\dot{v} \\
\ddot{N}\end{array}$ & ने & $\frac{n}{\text { กิ }}$ & & \\
\hline & & in & $\begin{array}{l}\infty \\
\stackrel{\lambda}{N}\end{array}$ & $\stackrel{0}{N}$ & $\stackrel{+}{\stackrel{\sim}{\sim}}$ & ๑ & $\ddot{n}$ & & \\
\hline & & เที & $\stackrel{0}{0}$ & $\equiv$ & ֻ̊. & $\widehat{\sigma}$ & $\stackrel{n}{\sim}$ & & \\
\hline & & เี & $\tilde{a}$ & $\Xi$ & तิ & $\Xi$ & ते & & \\
\hline & & $\bar{n}$ & 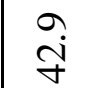 & $F$ & $\ddot{\sigma}$ & $\bar{\sigma}$ & $\underset{\infty}{\infty}$ & & \\
\hline & & $\frac{\pi}{n}$ & $\stackrel{0}{0}$ & 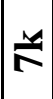 & $\begin{array}{l}0 \\
\dot{0}\end{array}$ & $\stackrel{y}{\alpha}$ & $\stackrel{\infty}{\sim}$ & & \\
\hline & & iñ & $\frac{0}{\hat{N}}$ & $i^{2}$ & $\begin{array}{l}\dot{+} \\
\dot{d}\end{array}$ & $\ddot{\sigma}$ & $\frac{\dot{\nabla}}{\Delta}$ & $\exists$ & $\overrightarrow{6}$ \\
\hline & & in & $\vec{m}$ & $i$ & $\begin{array}{l}0 \\
\ddot{n} \\
\ddot{d}\end{array}$ & $\ddot{\sigma}$ & $\frac{N}{m}$ & $\exists$ & ஜ̊ \\
\hline & & $\frac{1}{10}$ & $\begin{array}{l}0 \\
\ddot{n}\end{array}$ & $\frac{1}{N}$ & $\stackrel{0}{\circ}$ & ล์ & $\dot{\vec{v}}$ & $\equiv$ & $\stackrel{0}{0}$ \\
\hline & & 800 & ָ̃ & 20 & $\stackrel{\nabla}{\dot{m}}$ & 60 & $\stackrel{\varrho}{\varrho}$ & $\stackrel{\theta 0}{=}$ & $\begin{array}{l}0 \\
\dot{d}\end{array}$ \\
\hline & & 15 & $\stackrel{\nabla}{\stackrel{\nabla}{\sim}}$ & $\pi$ & $\stackrel{n}{n}$ & ă & $\stackrel{n}{m}$ & $\Xi$ & $\begin{array}{l}\infty \\
\dot{+}\end{array}$ \\
\hline$\ddot{m}$ & $\stackrel{0}{0}$ & $\mid \begin{array}{r}0 \\
\mid\end{array}$ & $\stackrel{n}{\text { n̊ }}$ & $\stackrel{0}{2}$ & ฉุ & $\Xi$ & $\stackrel{\infty}{\stackrel{\infty}{\infty}}$ & $\stackrel{\ominus}{=}$ & $\tilde{a}$ \\
\hline$\vec{m}$ & $\hat{0}$ & in & กุ & $\pi$ & $\ddot{a}$ & 8 & $\hat{m}$ & 크 & $\begin{array}{l}0 \\
\text { ì }\end{array}$ \\
\hline ల్ & 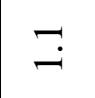 & in & aे & 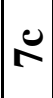 & $\stackrel{\sim}{\stackrel{j}{m}}$ & a & $\stackrel{n}{n}$ & $\stackrel{0}{=}$ & $\begin{array}{l}n \\
0 \\
n\end{array}$ \\
\hline ले & $m$ & $\frac{0}{10}$ & $\stackrel{a}{\sim}$ & 0 & 苘 & $\stackrel{2}{a}$ & $\stackrel{0}{\infty}$ & 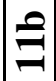 & $\stackrel{\infty}{a}$ \\
\hline ల్రా & $\stackrel{r}{\sim}$ & กี & 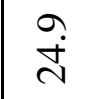 & $\boldsymbol{N}$ & $\stackrel{a}{\sigma}$ & ล์ & $\stackrel{0}{\infty}$ & $\stackrel{\Xi}{=}$ & $\vec{\lambda}$ \\
\hline 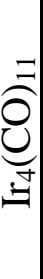 & 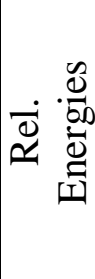 & 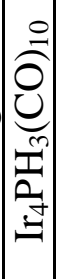 & 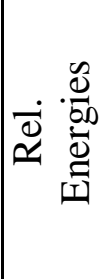 & 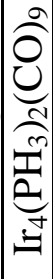 & 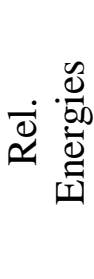 & 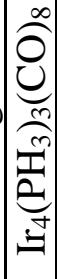 & 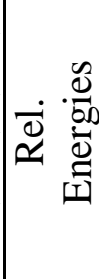 & 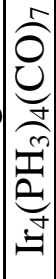 & 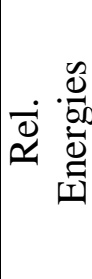 \\
\hline
\end{tabular}




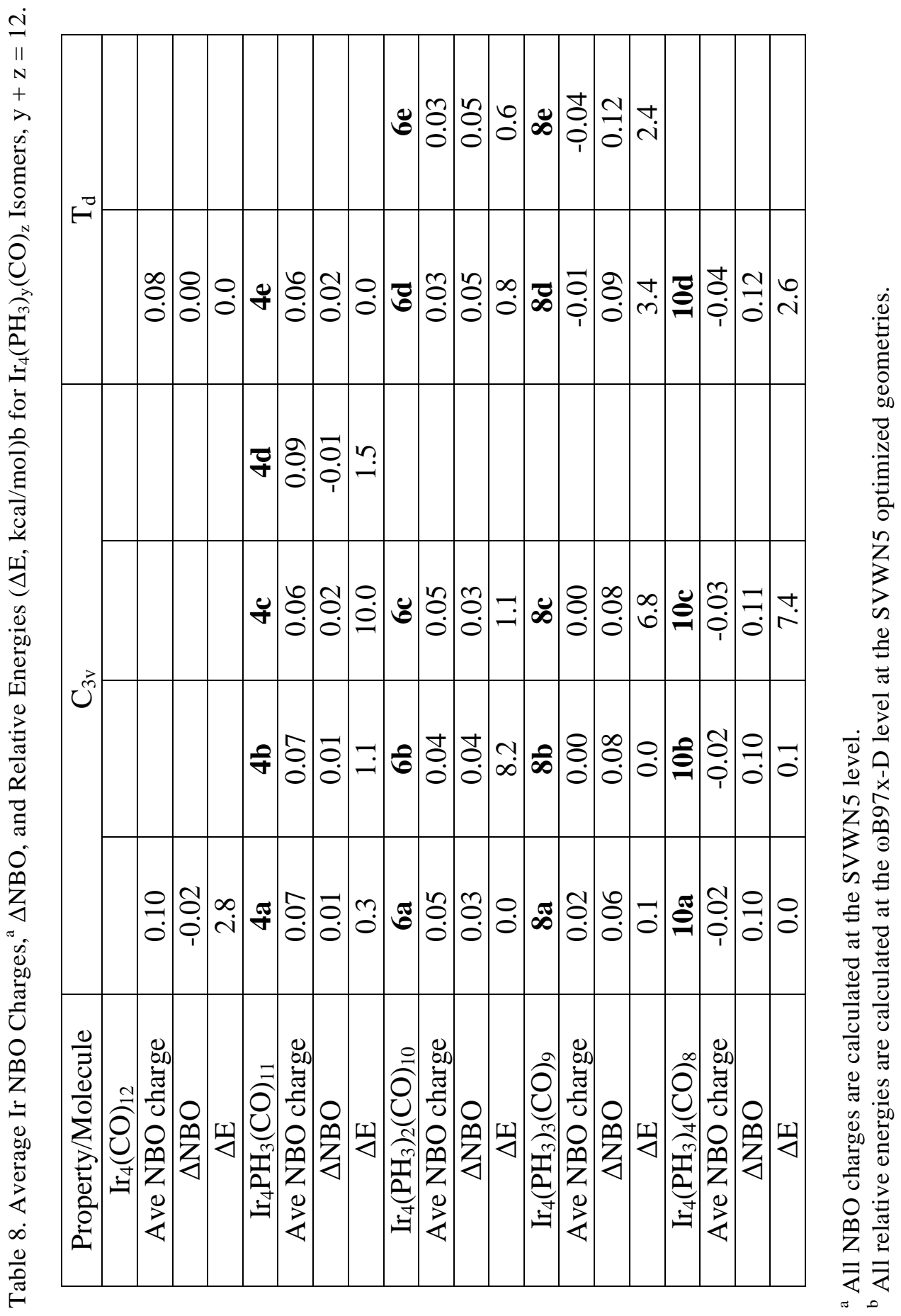


All of the predicted $\mathrm{H}$-atom transfer reactions occur on removal of a $\mathrm{CO}$ ligand from the cluster. The $\mathrm{H}$ atom that binds to the unsaturated $\mathrm{Ir}$ atom generated by $\mathrm{CO}$ removal comes from a $\mathrm{PH}_{3}$ bonded to an adjacent saturated $\mathrm{Ir}$ atom. If a terminal $\mathrm{PH}_{3}$ is directly bonded to the unsaturated Ir, it does not transfer an $\mathrm{H}$ atom. The $\mathrm{H}$ atom transfer reaction results in a cluster with a bridging phosphide group. Bridging $\mathrm{H}$ atoms are not found, although clusters with a bridging $\mathrm{H}$ atom are commonly observed experimentally. $[17,94,95]$ One possible reason for the lack of bridging $\mathrm{H}$ atoms is that the $\mathrm{H}$ atom needs to be a formal hydride in order to form an Ir-H-Ir bridge, as can be achieved experimentally by making the cluster an anion. For our neutral cluster, the $\mathrm{H}$ atom is unlikely to have very much hydridic character.

(a)

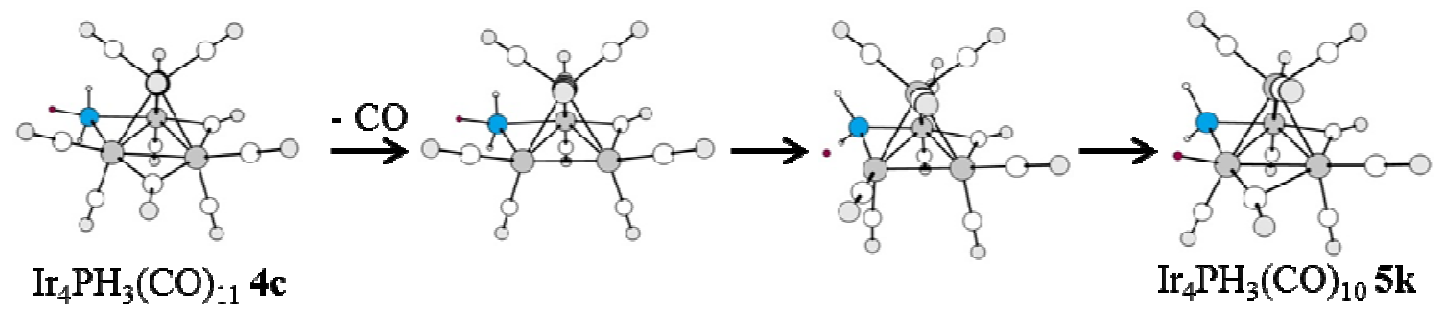

(b)

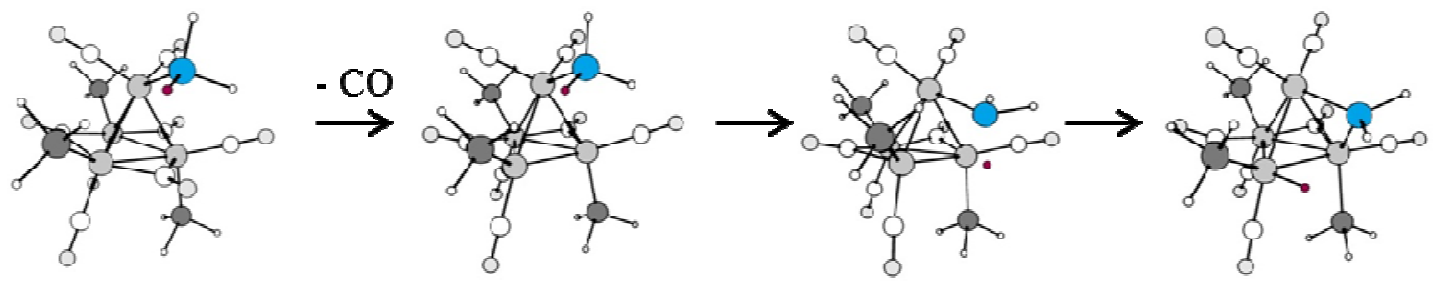

(c)

$$
\mathrm{Ir}_{4}\left(\mathrm{PH}_{3}\right)_{4}(\mathrm{CO})_{8} \text { 10a }
$$

$\mathrm{Ir}_{4}\left(\mathrm{PH}_{3}\right)_{4}(\mathrm{CO})_{7} \mathbf{1 1 b}$
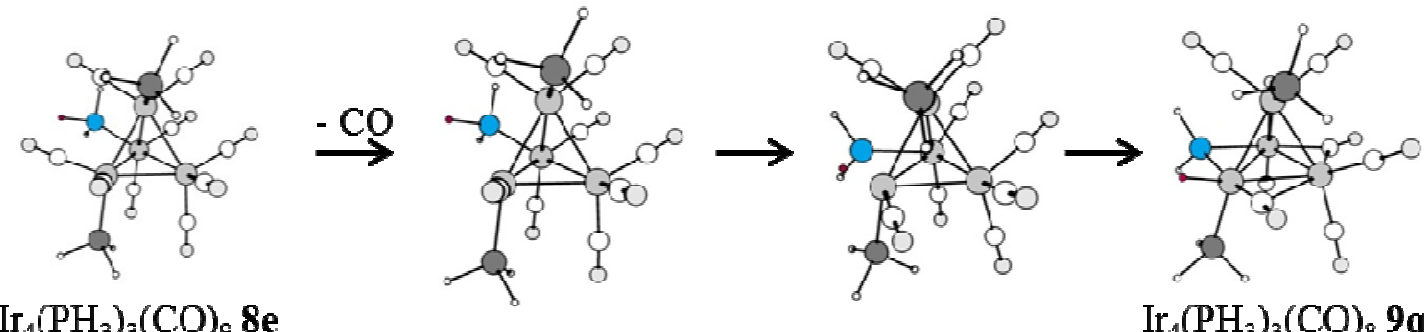

$\mathrm{Ir}_{4}\left(\mathrm{PH}_{3}\right)_{3}(\mathrm{CO})_{8} 9 \mathbf{q}$

Figure 5: Three types of $\mathrm{H}$ atom transfer reactions that occur on $\mathrm{CO}$ dissociation. The $\mathrm{H}$ and $\mathrm{P}$ atoms involved in the transfer reactions are marked in red and blue, respectively. The isomers in mid two columns are for description, not transition states or intermediates. 
(a)

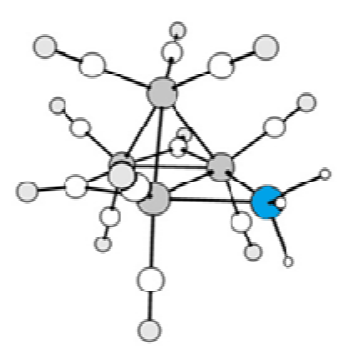

$\mathrm{Ir}_{4} \mathrm{PII}_{3}(\mathrm{CO})_{11} \mathbf{4 c}$

(b)

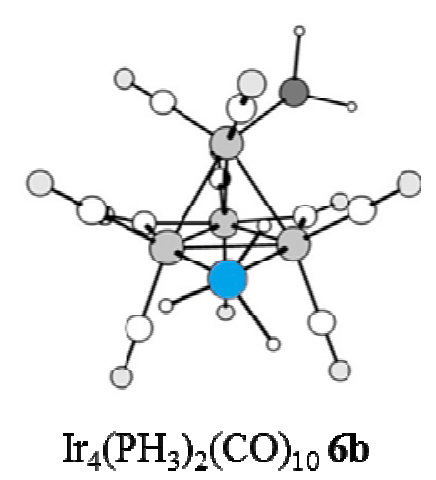

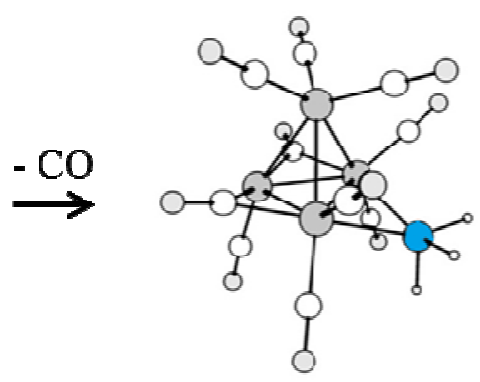

$\mathrm{Ir}_{4} \mathrm{PII}_{3}(\mathrm{CO})_{10} 5 \mathbf{l}$

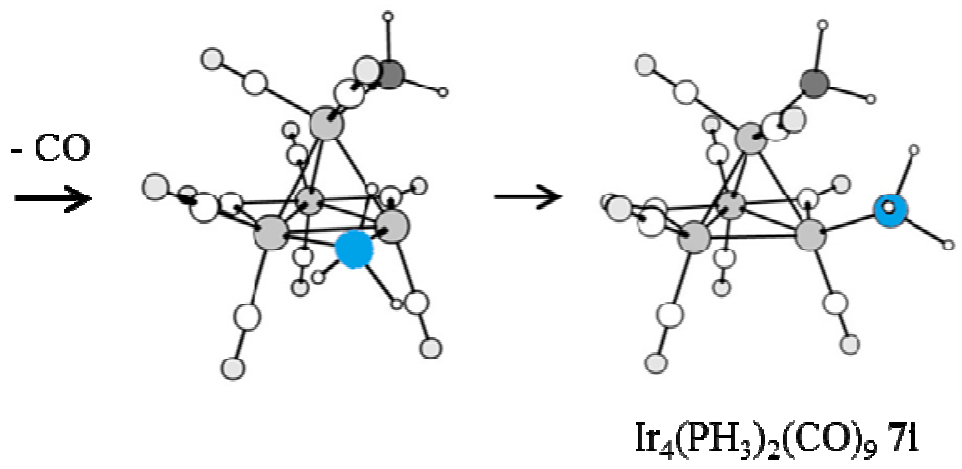

Figure 6: (a) A Bridging PH3 Maintained Process. (b) A Transformation from Bridging PH3 to Equatorial One. The $\mathrm{P}$ atoms involved in the transformation are marked in blue.

Another transformation involved in carbonyl dissociation involves changing the $\mathrm{PH}_{3}$ from a bridging site to an equatorial site. Figure 6a illustrates a case in which dissociation of an $e q \mathrm{CO}$ does not convert a bridging $\mathrm{PH}_{3}$ into a terminal one. When an $e q \mathrm{CO}$ that shares the Ir atom with the $\mathrm{PH}_{3}$ is removed, the $\mathrm{PH}_{3}$ leaves the bridging site and takes the place of the dissociated $e q \mathrm{CO}$ (Figure 6b).

The LDEs of $\mathrm{PH}_{3}$ and $\mathrm{CO}$ ligands of the $\mathrm{Ir}_{4}$ clusters are given in Table 9. (See also the Supporting Information, where they are arranged according to the reaction numbers in Figure 4.) The LDEs of carbonyls at various sites in the $\mathrm{C}_{3 \mathrm{v}}$ isomers follow the general order: $e q>a x>b r i>a p i$ for the lowest LDE in a group when $\mathrm{PH}_{3}$ does not change from a bri to an eq. The bri vs. api ordering is isomer-dependent, and so this order can switch, but clearly the $e q$ have the highest LDEs followed by the $a x$ LDEs. If the $\mathrm{PH}_{3}$ can rearrange to an $e q$ from a bri position, then the $e q \mathrm{CO}$ ligands have the lowest LDE. The hydrogen atom transfer processes that can occur when $\mathrm{CO}$ is removed lead to a lower energy product cluster, and so the CO LDEs decrease by 8 to $\sim 33 \mathrm{kcal} / \mathrm{mol}$.

As $\mathrm{PH}_{3}$ ligands are substituted for $\mathrm{CO}$, there is not a clear trend in the CO LDEs for the lowest energy species up to $\mathrm{x}=3$. The specific details regarding which $\mathrm{CO}$ will be lost are complicated by the similar energies of the various $\mathrm{C}_{3 \mathrm{v}}$ (and even $\mathrm{T}_{\mathrm{d}}$ ) isomers. For $\mathrm{x}=4$, the lowest-energy CO LDE is clearly greater than those characterizing the other low-energy isomers. We emphasize that these values are adiabatic thermodynamic values going to the lowest-energy product cluster.[86] Experimentally, as observed for much bulkier phosphines, the decarbonylation rates increase with increasing numbers of phosphines.[96] Our results are indeed consistent with these 
observations, because of the small steric bulk of $\mathrm{PH}_{3}$ and the fact that kinetic control may also pertain if there is an additional barrier to decarbonylation above that for the LDE. If carbonyl dissociation does occur with an additional energy barrier by an $\mathrm{S}_{\mathrm{N} 1}$ process,[97] the transition state will be of lower energy if there is a strong $\sigma$-donor in the cluster that increases the electron density on the Ir core. Such a transition state influence has been assumed for mononuclear complexes[98] and was used as a basis for inferring ligand dissociation rates for bi- and higher-nuclearity iridium clusters.[96] For $\operatorname{Ir}_{\mathrm{x}}\left(\mathrm{PH}_{3}\right)_{\mathrm{y}}(\mathrm{CO})_{\mathrm{z}}$ clusters, this reasoning would suggest that decarbonylation will take place at an increasing rate as more phosphines are substituted, in agreement with what has been reported.[42]

Given that the api and bri sites in the $\mathrm{C}_{3 \mathrm{v}}$ structures are comparable, the following question arises: at which site would ligand dissociation likely have the highest reaction rate? Our calculations predict NBO charges of the eq, ax, api, and bri carbonyls (charge on $\mathrm{C}$ plus the charge on $\mathrm{O}$ ) in $\mathrm{C}_{3 \mathrm{v}} \mathrm{Ir}_{4}(\mathrm{CO})_{12}$ to be $0.07 \mathrm{e}, 0.06 \mathrm{e},-0.02 \mathrm{e}$, and -0.25 e, respectively, with a total charge on the $\mathrm{Ir}_{4}$ cluster of $0.41 \mathrm{e}$. The corresponding charges on the $\mathrm{Ir}_{4}$ core in $\operatorname{Ir}_{4}(\mathrm{CO})_{11}$ generated after removal of an eq, ax, api, or bri $\mathrm{CO}$ are $0.52 \mathrm{e}, 0.40 \mathrm{e}, 0.40 \mathrm{e}$, and $0.33 \mathrm{e}$, respectively. This comparison suggests that removal of a bri CO leads to the largest increase in the electron density of the Ir core. Thus, we suggest, if a transition state for $\mathrm{CO}$ loss is present, that such a transition state would have the lowest energy for a bri CO. This inference is consistent with the experimental result that bridging carbonyls dissociate more readily in thermal decarbonylation.[42] Because bri $\mathrm{CO}$ ligands and $e q$, ax $\mathrm{CO}$ ligands can be readily interconverted,[40] substitution with a new ligand can take place at any basal position determined by its bulk and orbital direction; api substitutions are rare except through some selectively reactive decarbonylations that lower the activation energy, as they are not readily interconverted.[43]

The eq CO position has very high LDEs, ranging from $~ 51$ to $60 \mathrm{kcal} / \mathrm{mol}$, except for dissociation involving the transformation of $\mathrm{PH}_{3}$ from bri to eq, which lowers the LDE to $\sim 37 \mathrm{kcal} / \mathrm{mol}$, because the product is of lower energy. Different from the $\mathrm{PH}_{3}$ LDEs discussed below, the other bri CO LDEs, from $\sim 39$ to $\sim 46$ $\mathrm{kcal} / \mathrm{mol}$, are close to or even slightly greater than those of api $\mathrm{CO}$, ranging from 37 to $\sim 47 \mathrm{kcal} / \mathrm{mol}$. This result suggests that carbonyls can bind to two $\mathrm{Ir}$ atoms more efficiently than $\mathrm{PH}_{3}$. The LDEs of $\mathrm{CO}$ in the $\mathrm{T}_{\mathrm{d}}$ isomers, from $\sim 38$ to 44 $\mathrm{kcal} / \mathrm{mol}$, are close to those for api $\mathrm{CO}$ (the same trend was found for $\mathrm{PH}_{3} \mathrm{LDEs}$ ) and bri $\mathrm{CO}$ in the $\mathrm{C}_{3 \mathrm{v}}$ isomers.

For $\mathrm{PH}_{3}$ in the $\mathrm{C}_{3 \mathrm{v}}$ isomers, the LDEs range from $\sim 29$ to $\sim 51 \mathrm{kcal} / \mathrm{mol}$. The $e q$ $\mathrm{PH}_{3}$ ligands have the highest LDEs, from $\sim 45$ to $\sim 51 \mathrm{kcal} / \mathrm{mol}$, much higher than others by at least $\sim 10 \mathrm{kcal} / \mathrm{mol}$. In the remainder, the $a x \mathrm{PH}_{3}$ 's have LDEs from 35 to $\sim 41 \mathrm{kcal} / \mathrm{mol}$, followed by api LDEs from $\sim 32$ to $\sim 37 \mathrm{kcal} / \mathrm{mol}$. As a consequence of the higher energies of the bri $\mathrm{PH}_{3}$ isomers, their LDEs are the lowest, $\sim 30 \mathrm{kcal} / \mathrm{mol}$. The range of $\mathrm{PH}_{3} \mathrm{LDEs}$ in the $\mathrm{T}_{\mathrm{d}}$ structure, from $\sim 33$ to $\sim 38$ $\mathrm{kcal} / \mathrm{mol}$, is relatively narrow with respect to those in the $\mathrm{C}_{3 \mathrm{v}}$ isomer. Even if the product changes to the $\mathrm{C}_{3 \mathrm{v}}$ structure, the LDEs of the $\mathrm{T}_{\mathrm{d}} \mathrm{PH}_{3}$ ligands are very close to the api values. This result can be explained by the similar environment of the api Ir atom and the Ir atoms in the $\mathrm{T}_{\mathrm{d}}$ isomers, both of which are three-fold ligand 


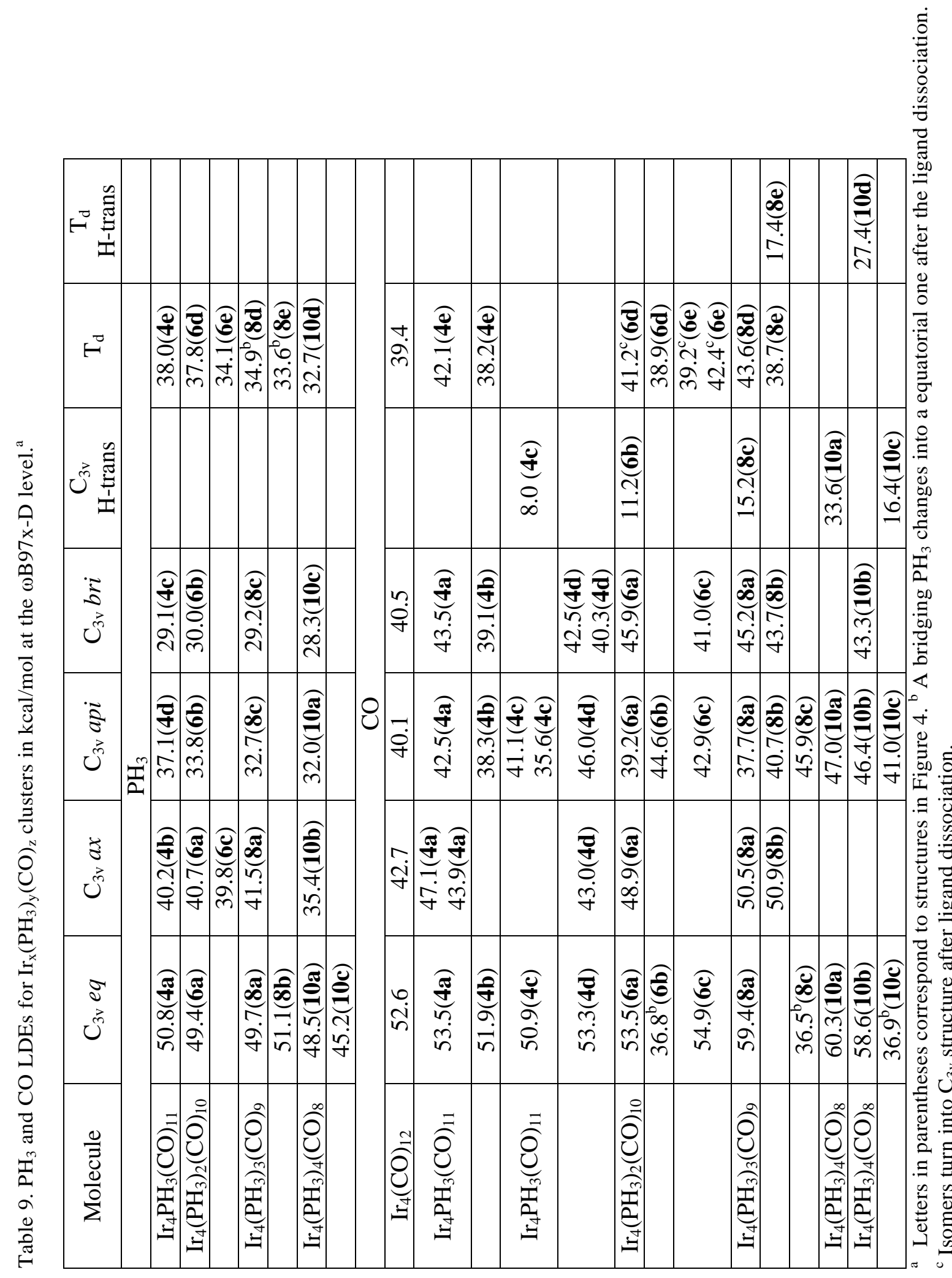


coordinated. As more carbonyls are replaced, the LDEs gradually decrease, similar to the trend found in $\operatorname{Ir}\left(\mathrm{PH}_{3}\right)_{\mathrm{y}}(\mathrm{CO})_{\mathrm{z}}$ and $\operatorname{Ir}_{2}\left(\mathrm{PH}_{3}\right)_{\mathrm{y}}(\mathrm{CO})_{\mathrm{z}}$ clusters.

\section{Conclusions}

Calculations of the structures and energetics of a range of $\operatorname{Ir}_{x}\left(\mathrm{PH}_{3}\right)_{\mathrm{y}}(\mathrm{CO})_{\mathrm{z}}(\mathrm{x}=1,2$, 4) have been performed. Benchmark calculations for the LDEs for the $x=1$ and 2 clusters were performed at the DFT level with 10 exchange-correlation functionals as well as at the correlated molecular orbital MP2 and CCSD(T) levels. The best agreement with CCSD(T) was found by using the $\omega \mathrm{B} 97 \mathrm{X}-\mathrm{D}$ functional so it was used for the $x=4 \mathrm{LDE}$ calculations. The doublet states of the $\operatorname{Ir}\left(\mathrm{PH}_{3}\right)_{y}(\mathrm{CO})_{z}$ clusters are significantly lower in energy than the quartet states. The carbonyls are stronger $\pi$-electron acceptor ligands than $\mathrm{PH}_{3}$, and the $\mathrm{PH}_{3}$ ligands act as stronger $\sigma$-electron donors and weaker $\pi$-electron acceptors than $\mathrm{CO}$. Such electronic effects strengthen the Ir-C bond and increase the LDEs of the CO ligand for the complexes with a single Ir atom. The $\sigma$-electron donation ability of $\mathrm{PH}_{3}$ provides more electron density on the Ir atom that can be used for $\pi$-back-bonding to the carbonyl. Yet this increased $\sigma$-electron donation leads to an increased repulsion with the $\mathrm{d}$ valence electrons from the Ir (often called Pauli repulsion). The balance of these two effects will govern the properties at the minimum for the mononuclear iridium complexes, and it is also relevant to the LDE if the properties of the Ir-containing product are similar. The trans influence of carbonyls is important in determining the structure, relative energies, and dissociation energies of these mononuclear iridium complexes. The isomers of these complexes with ax phosphines are of lower energy. The Ir-CO LDE increases with increasing substitution of $\mathrm{CO}$ by $\mathrm{PH}_{3}$ ligands, whereas the LDEs of $\mathrm{PH}_{3}$ ligands decrease slightly as more $\mathrm{PH}_{3}$ ligands are added. $\mathrm{IrL}_{4}$ complexes are more distorted, with lower LDEs, than $\operatorname{IrL}_{3}$, and steric effects also play roles even in these small complexes.

The three basic structures of $\operatorname{Ir}_{2}\left(\mathrm{PH}_{3}\right)_{\mathrm{y}}(\mathrm{CO})_{\mathrm{z}}$ isomers have $\mathrm{C}_{2 \mathrm{v}}, \mathrm{C}_{2}$, and $\mathrm{D}_{3 \mathrm{~d}}$ symmetry. The two trans ligands along the $\mathrm{Ir}-\mathrm{Ir}$ axis in the $\mathrm{D}_{3 \mathrm{~d}}$ isomers are strongly bonded, leading to lower energy structures. The dissociation of such ligands results in higher energy isomers. $\mathrm{C}_{2}$ and $\mathrm{C}_{2 \mathrm{v}}$ are coordinatively saturated isomers with energies that are close to each other, whereas the $\mathrm{C}_{2}$ isomers of coordinatively unsaturated species are of lower energy. The changes are similar to those found for $\operatorname{Ir}\left(\mathrm{PH}_{3}\right)_{y}(\mathrm{CO})_{z}$ complexes as more $\mathrm{PH}_{3}$ ligands are introduced. $\mathrm{PH}_{3}$ substitution increases the negative charge on the Ir atom and strengthens the binding between the carbonyls and the Ir atom and slightly decreases the LDEs of the $\mathrm{PH}_{3}$ ligands. This trend is the same as that predicted for $\operatorname{Ir}\left(\mathrm{PH}_{3}\right)_{y}(\mathrm{CO})_{\mathrm{z}}$ complexes.

The $\mathrm{C}_{3 \mathrm{v}}$ structures of $\operatorname{Ir}_{4}\left(\mathrm{PH}_{3}\right)_{\mathrm{y}}(\mathrm{CO})_{\mathrm{z}}$ clusters have similar energies with respect to the $\mathrm{T}_{\mathrm{d}}$ isomers for $\mathrm{Ir}_{4} \mathrm{PH}_{3}(\mathrm{CO})_{11}$ and $\mathrm{Ir}_{4}\left(\mathrm{PH}_{3}\right)_{2}(\mathrm{CO})_{10}$, and the $\mathrm{C}_{3 \mathrm{v}}$ structures are of even lower energy for $\operatorname{Ir}_{4}\left(\mathrm{PH}_{3}\right)_{3}(\mathrm{CO})_{9}$ and $\operatorname{Ir}_{4}\left(\mathrm{PH}_{3}\right)_{4}(\mathrm{CO})_{8}$. This conclusion is consistent with observations of $\mathrm{C}_{3 \mathrm{v}}$ structures for mono-phosphine $\mathrm{Ir}_{4}$ clusters with larger and more electron-withdrawing $\mathrm{R}$ groups on the phosphorus.[40] Our explanation for these energy differences is that the charge differences on the Ir atoms do not vary much from those in the $\operatorname{Ir}_{4}(\mathrm{CO})_{12} \mathrm{~T}_{\mathrm{d}}$ structure and that steric interactions are minimized. Structural transformations in the process of carbonyl dissociation can occur with $\mathrm{T}_{\mathrm{d}}$ isomers converting into $\mathrm{C}_{3 \mathrm{v}}$ isomers; the reverse does not occur. Hydrogen atom transfer from a $\mathrm{PH}_{3}$ usually occurs with a bridging carbonyl 
dissociation coupled with a weakly bonded $\mathrm{PH}_{3}$ and results in a bridging phosphido group and an $\mathrm{Ir}-\mathrm{H}$ bond that makes the cluster coordinatively saturated. A bridging $\mathrm{PH}_{3}$ can move to an equatorial position as that $e q$ carbonyl is dissociated. Such a transformation stabilizes the product cluster and reduces the LDEs. When hydrogen atom transfer and the rearrangement of the $\mathrm{PH}_{3}$ from a bri to an $e q$ position does not occur, the lowest CO LDE for a given site follows the order $e q>a x>b r i>a p i$. Up to $\mathrm{x}=3$, the lowest energy $\mathrm{CO}$ LDEs are approximately the same as $\mathrm{PH}_{3}$ ligands are substituted for CO. Experimentally, an increase in the numbers of phosphines leads to an increase in the decarbonylation rates,[42] most likely a consequence of the use of much bulkier phosphines and potentially kinetic control of the decarbonylation. The addition of the phosphines makes the Ir atoms more electron rich, leading, it has been hypothesized, to a lower-energy transition state for thermal decarbonylation.[96] The NBO calculations for the $\mathrm{C}_{3 \mathrm{v}}$ isomers show that the bridging carbonyls donate less electron density to the tetrairidium core (are less positive) than do the terminal carbonyls. The transition state for dissociation of a bridging carbonyl has more electron density on the tetrairidium core than those involving carbonyls in other sites, as shown by the charge distributions in the respective $\operatorname{Ir}_{4}(\mathrm{CO})_{11}$ clusters. Thus, one should expect lower-energy transition states for the dissociation of bridging carbonyls. Because CO ligands can switch between the basal and bridging sites,[40] basal substitution can occur more readily than apical substitution.[42] Similar to the trend found for $\operatorname{Ir}\left(\mathrm{PH}_{3}\right)_{y}(\mathrm{CO})_{z}$ and $\operatorname{Ir}_{2}\left(\mathrm{PH}_{3}\right)_{y}(\mathrm{CO})_{z}$, the LDEs of the $\mathrm{PH}_{3}$ 's in the tetra-iridium cluster gradually decrease with an increasing number of $\mathrm{PH}_{3}$ 's.

\section{Acknowledgements}

The authors are grateful to the U.S. Department of Energy (DOE), Office of Science, Basic Energy Sciences, under contract DE-SC0005822, for financial support. Some of the computational work was performed at the Molecular Science Computing Facility, William R. Wiley Environmental Molecular Sciences Laboratory, a national scientific user facility sponsored by the DOE Office of Biological and Environmental Research and located at Pacific Northwest National Laboratory (PNNL). PNNL is operated for DOE by Battelle Memorial Institute under Contract No. DE-AC06-76RLO-1830. We thank the Alabama Supercomputing Center for providing computational resources. D.A.D. thanks the Robert Ramsay Chair Fund of The University of Alabama for support.

\section{Notes and references}

[1] T. Shibata, Y. Kobayashi, S. Maekawa, N. Toshida, K. Takagi, Iridium-catalyzed enantioselective cycloisomerization of nitrogen-bridged 1,6-enynes to 3-azabicylo[4.1.0]heptenes, Tetrahedron, 61 (2005) 9018-9024.

[2] Y. Yamamoto, H. Hayashi, T. Saigoku, H. Nishiyama, Domino Coupling Relay Approach to Polycyclic Pyrrole-2-carboxylates, J. Am. Chem. Soc., 127 (2005) 10804-10805.

[3] D. Santi, T. Holl, V. Calemma, J. Weitkamp, High-performance ring-opening catalysts based on iridium-containing zeolite Beta in the hydroconversion of decalin, Appl. Catal. A: General, 455 (2013) 46-57. 
[4] D. Morales-Morales, R. Redón, Z. Wang, D.W. Lee, C. Yung, K. Magnuson, C.M. Jensen, Selective dehydrogenation of alcohols and diols catalyzed by a dihydrido iridium PCP pincer complex, Can. J. Chem., 79 (2001) 823-829.

[5] A. Nait Ajjou, First example of water-soluble transition-metal catalysts for Oppenauer-type oxidation of secondary alcohols, Tetrahedron Lett., 42 (2001) 13-15.

[6] T. Suzuki, T. Matsuo, K. Watanabe, T. Katoh, Iridium-Catalyzed Oxidative Dimerization of Primary Alcohols to Esters Using 2-Butanone as an Oxidant, Synlett, 2005 (2005) 1453-1455.

[ 7 ] P. Barbaro, C. Bianchini, P. Frediani, A. Meli, F. Vizza, Chemoselective oxidation of 3,5-di-tert-butylcatechol by molecular oxygen. Catalysis by an iridium(III) catecholate through its dioxygen adduct, Inorg. Chem., 31 (1992) 1523-1529.

[8] P. Barbaro, C. Bianchini, K. Linn, C. Mealli, A. Meli, F. Vizza, F. Laschi, P. Zanello, Dioxygen uptake and transfer by Co(III), Rh(III) and Ir(III) catecholate complexes, Inorg. Chim. Acta, 198-200 (1992) 31-56.

[9]S. Iwasa, A. Fakhruddin, H.S. Widagdo, H. Nishiyama, A Rapid and Efficient Synthesis of Quinone Derivatives: $\mathrm{Ru}(\mathrm{II})$ - or $\operatorname{Ir}(\mathrm{I})-\mathrm{Catalyzed} \mathrm{Hydrogen} \mathrm{Peroxide} \mathrm{Oxidation} \mathrm{of} \mathrm{Phenols} \mathrm{and} \mathrm{Methoxyarenes,} \mathrm{Adv.}$ Synth. Catal., 347 (2005) 517-520.

[10] X.-Q. Gu, W. Chen, D. Morales-Morales, C.M. Jensen, Dehydrogenation of secondary amines to imines catalyzed by an iridium PCP pincer complex: initial aliphatic or direct amino dehydrogenation?, J. Mol. Catal. A: Chem., 189 (2002) 119-124.

[11] W.H. Bernskoetter, M. Brookhart, Kinetics and Mechanism of Iridium-Catalyzed Dehydrogenation of Primary Amines to Nitriles, Organometallics, 27 (2008) 2036-2045.

[12] R. Crabtree, Iridium compounds in catalysis, Acc. Chem. Res., 12 (1979) 331-337.

[13] J.M. Brown, Directed Homogeneous Hydrogenation [New Synthetic Methods (65)], Angew. Chem. Int. Ed., 26 (1987) 190-203.

[14] H. Werner, Carbene-Transition Metal Complexes Formed by Double C-H Bond Activation, Angew. Chem. Int. Ed., 49 (2010) 4714-4728.

[15] R.J. Burford, W.E. Piers, M. Parvez, $\beta$-Elimination-Immune PCcarbeneP Iridium Complexes via Double C-H Activation: Ligand-Metal Cooperation in Hydrogen Activation, Organometallics, 31 (2012) 2949-2952.

[16] B.G. Hashiguchi, S.M. Bischof, M.M. Konnick, R.A. Periana, Designing Catalysts for Functionalization of Unactivated C-H Bonds Based on the CH Activation Reaction, Acc. Chem. Res., 45 (2012) 885-898.

[17] L. Malatesta, G. Caglio, Tetranuclear carbonyliridates, Chem. Comm., (1967) 420-421.

[18] R. Takeuchi, S. Tanaka, Y. Nakaya, Iridium complex-catalyzed [2+2+2] cycloaddition of $\alpha, \omega$-diynes with monoalkynes: a new and efficient catalyst for cyclotrimerization of alkynes, Tetrahedron Lett., 42 (2001) 2991-2994.

[19] E. Farnetti, N. Marsich, Regioselective cyclotrimerization of phenylacetylenes to 1,2,4-triarylbenzenes catalyzed by iridium-diphosphine complexes, J. Organomet. Chem., 689 (2004) 14-17.

[20] N. Jeong, D.H. Kim, J.H. Choi, Desymmetrization of meso-dienyne by asymmetric Pauson-Khand type reaction catalysts, Chem. Commun., (2004) 1134-1135.

[21] T. Shibata, N. Toshida, M. Yamasaki, S. Maekawa, K. Takagi, Iridium-catalyzed enantioselective Pauson-Khand-type reaction of 1,6-enynes, Tetrahedron, 61 (2005) 9974-9979.

[22] T. Shibata, K. Takasaku, Y. Takesue, N. Hirata, K. Takagi, Iridium Complex-Catalyzed Enantioselective Intramolecular [4+2] Cycloaddition of Dieneynes, Synlett, (2002) 1681-1682.

[23] T. Shibata, G. Nishizawa, K. Endo, Iridium-Catalyzed Enantioselective Formal [4+2] Cycloaddition of Biphenylene and Alkynes for the Construction of Axial Chirality, Synlett, (2008) 765-768.

[24] S. Fang, X. Liang, Y. Long, X. Li, D. Yang, S. Wang, C. Li, Iridium-Catalyzed Asymmetric Ring-Opening of Azabicyclic Alkenes with Phenols, Organometallics, 31 (2012) 3113-3118.

[25] A. Uzun, D.A. Dixon, B.C. Gates, Prototype Supported Metal Cluster Catalysts: Ir4 and Ir6, ChemCatChem, 3 (2011) 95-107.

[26] A.J.L Hanlan, G.A. Ozin, Synthesis using transition metal diatomic molecules. Dirhodium octacarbonyl and diiridium octacarbonyl, J. Am. Chem. Soc., 96 (1974) 6324-6329.

[27] A.J.L Hanlan, G. A. Ozin, Iridium atom chemistry: a reappraisal of the matrix synthesis of diiridium octacarbonyl, $\mathrm{Ir}_{2}(\mathrm{CO})_{8}$, J. Organomet. Chem., 179 (1979) 57-64.

[28] G.A. Ozin, A.J.L. Hanlan, Rhodium and iridium atom chemistry: binary carbonyls of rhodium and iridium, Inorg. Chem., 18 (1979) 2091-2101.

[29] Y. Shvo, R.M. Laine, Homogeneous catalytic activation of C-N bonds. Alkyl exchange between tertiary amines, J. Chem. Soc., Chem. Commun., (1980) 753-754.

[30] R.A. Schunn, G.C. Demitras, H.W. Choi, E.L. Muetterties, Methane formation in the reaction of carbon monoxide and hydrogen in the presence of iridium and osmium clusters, Inorg. Chem., 20 (1981) 4023-4025. 
[31] H.-K. Wang, H.W. Choi, E.L. Muetterties, Catalytic hydrogenation of carbon monoxide with dodecacarbonyltetrairidium and aluminum chloride, Inorg. Chem., 20 (1981) 2661-2663.

[32] T. Shido, T. Okazaki, M. Ichikawa, EXAFS/FT-IR characterization of tetra-iridium carbonyl clusters bound to tris-(hydroxymethyl)phosphine grafted silica surface catalytically active for propene oxidation to acetone, J. Mol. Catal. A: Chem., 120 (1997) 33-45.

[33] F.A. Cotton, G. Wilkinson, Advanced Inorganic Chemistry, 4th ed., John Wiley \& Sons, New York, 1980, 1050-1055.

[34] F.A. Cotton, G. Wilkinson, Advanced Inorganic Chemistry, 5th ed., John Wiley \& Sons, New York, 1988, 1023-1028.

[35] F. Cariati, V. Valenti, G. Zerbi, Structure of $\operatorname{Ir}_{4}(\mathrm{CO})_{12}$ from its vibrational spectrum, Inorg. Chim. Acta, 3 (1969) 378-382.

[36] M.R. Churchill, J.P. Hutchinson, Crystal structure of tetrairidium dodecacarbonyl, $\operatorname{Ir}_{4}(\mathrm{CO})_{12}$. An unpleasant case of disorder, Inorg. Chem., 17 (1978) 3528-3535.

[37] T.H. Walter, L. Reven, E. Oldfield, Magic-angle spinning carbon-13 NMR spectroscopy of transition-metal carbonyl clusters, J. Phys. Chem. 93 (1989) 1320-1326.

[38]D. Braga, F. Grepioni, J. J. Byrne, M. J. Calhorda, Molecular Structure and Crystal Organization of Neutral and Ionic Derivatives of [M4(CO)12] $(\mathrm{M}=\mathrm{Co}$, Rh or Ir) Clusters and a Bonding Study by Extended-Huckel Calculations, J. Chem. Soc. Dalton Trans. (1995) 3287-3296

[39] K. Besançon, G. Laurenczy, T. Lumini, R. Roulet, G. Gervasio, Solution Equilibria in Trialkyl-Phosphite Derivatives of $\left[\operatorname{Ir}_{4}(\mathrm{CO})_{12}\right]$. Crystal Structure of $\left[\operatorname{Ir}_{4}(\mathrm{CO})_{11}\left\{\mathrm{P}\left(\mathrm{OCH}_{2}\right)_{3} \mathrm{CEt}\right\}\right]$, Helv. Chim. Acta, 76 (1993) 2926-2935.

[40] R. Ros, A. Scrivanti, V.G. Albano, D. Braga, L. Garlaschelli, Chemistry of tetrairidium carbonyl clusters. Part 1. Synthesis, chemical characterization, and nuclear magnetic resonance study of mono- and di-substituted phosphine derivatives. X-Ray crystal structure determination of the diaxial isomer of $\left[\mathrm{Ir}_{4}(\mathrm{CO})_{7}(\mu-\mathrm{CO})_{3}\left(\mathrm{Me}_{2} \mathrm{PCH}_{2} \mathrm{CH}_{2} \mathrm{PMe}_{2}\right)\right]$, J. Chem. Soc., Dalton Trans., (1986) 2411-2421.

[41] G.F. Stuntz, J.R. Shapley, Direct synthesis of mono- and disubstituted phosphorus ligand derivatives of dodecacarbonyltetrairidium, Inorg. Chem., 15 (1976) 1994-1996.

[42] A. Okrut, O. Gazit, N.d. Silva, R. Nichiporuk, A. Solovyov, A. Katz, Stabilization of coordinatively unsaturated Ir4 clusters with bulky ligands: a comparative study of chemical and mechanical effects, Dalton Trans., 41 (2012) 2091-2099.

[43] A. Okrut, R.C. Runnebaum, X. Ouyang, J. Lu, C. Aydin, S.-J. Hwang, S. Zhang, O.A. Olatunji-Ojo, K.A. Durkin, D.A. Dixon, B.C. Gates, A. Katz, Selective molecular recognition by nanoscale environments in a supported iridium cluster catalyst, Nat Nano, 9 (2014) 459-465.

[44] P. Hohenberg, W. Kohn, Inhomogeneous Electron Gas, Phys. Rev., 136 (1964) B864-B871.

[45] W. Kohn, L.J. Sham, Self-Consistent Equations Including Exchange and Correlation Effects, Phys. Rev., 140 (1965) A1133-A1138.

[46] R.G. Parr, W. Yang, Density-functional theory of atoms and molecules, Oxford University Press, Oxford, 1989.

[47] G. D. Purvis, III, R. J. Bartlett, A Full Coupled Cluster Singles and Doubles Model: The Inclusion of Disconnected Triples. J. Chem. Phys. 76 (1982) 1910-1918.

[48] K. Raghavachari, G. W. Trucks, J. A. Pople, M. Head-Gordon, A Fifth-Order Perturbation Comparison of Electron Correlation Theories. Chem. Phys. Lett. 157 (1989) 479-483.

[49] J. D. Watts, J. Gauss, R. J. Bartlett, Coupled-Cluster Methods with Noniterative Triple Excitations for Restricted Open Shell Hartree-Fock and Other General Single Determinant Reference Functions. Energies and Analytical Gradients. J. Chem. Phys. 98 (1993) 8718-8733.

[50] R.J. Bartlett, M. Musiał, Coupled-cluster theory in quantum chemistry, Rev. Mod. Phys. 79 (2007) 291-352.

[51] M. Chen, J.E. Dyer, B.C. Gates, A. Katz, D.A. Dixon, Structures and Stability of Irn(CO)m, Mol. Phys., 110 (2012) 1977-1992.

[52] J.C. Slater, The Self-Consistent Field for Molecular and Solids, Quantum Theory of Molecular and Solids, McGraw-Hill, New York, 1974.

[53] S.H. Vosko, L. Wilk, M. Nusair, Accurate spin-dependent electron liquid correlation energies for local spin density calculations: a critical analysis, Can. J. Phys. 58 (1980) 1200-1211.

[54] T.H. Dunning, Gaussian basis sets for use in correlated molecular calculations. I. The atoms boron through neon and hydrogen, J. Chem. Phys. 90 (1989) 1007-1023.

[55] R.A. Kendall, T.H. Dunning, R.J. Harrison, Electron affinities of the first-row atoms revisited. Systematic basis sets and wave functions, J. Chem. Phys. 96 (1992) 6796-6806. 
[56] D. Figgen, K.A. Peterson, M. Dolg, H. Stoll, Energy-consistent pseudopotentials and correlation consistent basis sets for the 5d elements Hf-Pt, J. Chem. Phys. 130 (2009) 164108-164112.

[57] A.D. Becke, Density-functional thermochemistry. III. The role of exact exchange, J. Chem. Phys. 98 (1993) 5648-5652.

[58] C. Lee, W. Yang, R.G. Parr, Development of the Colle-Salvetti correlation-energy formula into a functional of the electron density, Phys. Rev. B, 37 (1988) 785-789.

[59] S. Grimme, Semiempirical GGA-type density functional constructed with a long-range dispersion correction, J. Comp. Chem. 27 (2006) 1787-1799.

[60] A.D. Becke, Density-functional thermochemistry. V. Systematic optimization of exchange-correlation functionals, J. Chem. Phys. 107 (1997) 8554-8560.

[61] H.L. Schmider, A.D. Becke, Optimized density functionals from the extended G2 test set, J. Chem. Phys. 108 (1998) 9624-9631.

[62] A.D. Becke, Density-functional exchange-energy approximation with correct asymptotic behavior, Phys. Rev. A, 38 (1988) 3098-3100.

[63 ] T. Yanai, D.P. Tew, N.C. Handy, A new hybrid exchange-correlation functional using the Coulomb-attenuating method (CAM-B3LYP), Chem. Phys. Lett. 393 (2004) 51-57.

[64] Y. Zhao, D. Truhlar, The M06 suite of density functionals for main group thermochemistry, thermochemical kinetics, noncovalent interactions, excited states, and transition elements: two new functionals and systematic testing of four M06-class functionals and 12 other functionals, Theor Chem Account, 120 (2008) 215-241.

[65] J.P. Perdew, Y. Wang, Accurate and simple analytic representation of the electron-gas correlation energy, Phys. Rev. B, 45 (1992) 13244-13249.

[66] J.P. Perdew, K. Burke, Y. Wang, Generalized gradient approximation for the exchange-correlation hole of a many-electron system, Phys. Rev. B, 54 (1996) 16533-16539.

[67] K. Burke, J.P. Perdew, Y. Wang, in Electronic Density Functional Theory: Recent Progress and New Directions, Plenum, New York, 1997.

[68] J.P. Perdew, K. Burke, M. Ernzerhof, Generalized Gradient Approximation Made Simple, Phys. Rev. Lett. 77 (1996) 3865-3868.

[69] J.P. Perdew, K. Burke, M. Ernzerhof, Generalized Gradient Approximation Made Simple [Phys. Rev. Lett. 77, 3865 (1996)], Phys. Rev. Lett. 78 (1997) 1396-1396.

[70] J.-D. Chai, M. Head-Gordon, Systematic optimization of long-range corrected hybrid density functionals, J. Chem. Phys. 128 (2008) 084106.

[71] J.-D. Chai, M. Head-Gordon, Long-range corrected hybrid density functionals with damped atom-atom dispersion corrections, Physical Chemistry Chemical Physics, 10 (2008) 6615-6620.

[72] P. J. Knowles, C. Hampel, H.-J. Werner, Coupled Cluster Theory for High Spin, Open Shell Reference Wave Functions. J. Chem. Phys. 99 (1993) 5219-5227.

[73] P. J. Knowles, C. Hampel, H.-J. Werner Erratum: Coupled Cluster Theory for High Spin, Open Shell Reference Wave Functions. [J. Chem. Phys. 99, 5219 (1993)]. J. Chem. Phys. 112 (2000) 3106-3107.

[74] M. J. O. Deegan, P. J. Knowles, Perturbative Corrections to Account for Triple Excitations in Closed and Open Shell Coupled Cluster Theories. Chem. Phys. Lett. 227 (1994) 321-327.

[75] R. D. Amos, J. S. Andrews, N. C. Handy, P. J. Knowles, Open-shell Møller-Plesset Perturbation Theory. Chem. Phys. Lett. 185 (1991) 256-264

[76] M.J. Frisch, G.W. Trucks, H.B. Schlegel, G.E. Scuseria, M.A. Robb, J.R. Cheeseman, G. Scalmani, V. Barone, B. Mennucci, G.A. Petersson, H. Nakatsuji, M. Caricato, X. Li, H.P. Hratchian, A.F. Izmaylov, J. Bloino, G. Zheng, J.L. Sonnenberg, M. Hada, M. Ehara, K. Toyota, R. Fukuda, J. Hasegawa, M. Ishida, T. Nakajima, Y. Honda, O. Kitao, H. Nakai, T. Vreven, J. Montgomery, J. A., J.E. Peralta, F. Ogliaro, M. Bearpark, J.J. Heyd, E. Brothers, K.N. Kudin, V.N. Staroverov, R. Kobayashi, J. Normand, K. Raghavachari, A. Rendell, J.C. Burant, S.S. Iyengar, J. Tomasi, M. Cossi, N. Rega, N.J. Millam, M. Klene, J.E. Knox, J.B. Cross, V. Bakken, C. Adamo, J. Jaramillo, R. Gomperts, R.E. Stratmann, O. Yazyev, A.J. Austin, R. Cammi, C. Pomelli, J.W. Ochterski, R.L. Martin, K. Morokuma, V.G. Zakrzewski, G.A. Voth, P. Salvador, J.J. Dannenberg, S. Dapprich, A.D. Daniels, Ö. Farkas, J.B. Foresman, J.V. Ortiz, J. Cioslowski, D.J. Fox, Gaussian 09, Revision D.1, Gaussian, Inc., Wallingford CT, 2009.

[77] P.J. Knowles, F.R. Manby, M. Schütz, P. Celani, G. Knizia, T. Korona, R. Lindh, A. Mitrushenkov, G. Rauhut, T.B. Adler, R.D. Amos, A. Bernhardsson, A. Berning, D.L. Cooper, M.J.O. Deegan, A.J. Dobbyn, F. Eckert, E. Goll, C. Hampel, A. Hesselmann, G. Hetzer, T. Hrenar, G. Jansen, C. Köppl, Y. Liu, A.W. Lloyd, R.A. Mata, A.J. May, S.J. McNicholas, W. Meyer, M.E. Mura, A. Nicklass, P. Palmieri, K. Pflüger, R. Pitzer, M. 
Reiher, T. Shiozaki, H. Stoll, A.J. Stone, R. Tarroni, T. Thorsteinsson, M. Wang, A. Wolf, MOLPRO, version 2010.1, a package of ab initio programs, http://www.molpro.net, 2010.

[78] M.J.S. Dewar, A review of $\pi$ Complex Theory, Bull. Soc. Chim. Fr., 18 (1951) C71-C79.

[79] J. Chatt, L.A. Duncanson, 586. Olefin co-ordination compounds. Part III. Infra-red spectra and structure: attempted preparation of acetylene complexes, J. Chem. Soc. (1953) 2939-2947.

[80] M. Nyberg, A. Föhlisch, L. Triguero, A. Bassan, A. Nilsson, L.G.M. Pettersson, Bonding in metal-carbonyls: A comparison with experiment and calculations on adsorbed CO, J. Molec. Struct.: THEOCHEM, 762 (2006) 123-132.

[81] J.P. Foster, F. Weinhold, Natural hybrid orbitals, J. Am. Chem. Soc. 102 (1980) 7211-7218.

[82] A.E. Reed, L.A. Curtiss, F. Weinhold, Intermolecular interactions from a natural bond orbital, donor-acceptor viewpoint, Chem. Rev. 88 (1988) 899-926.

[83] E. D. Glendening, J. K. Badenhoop, A. E. Reed, J. E. Carpenter, J. A Bohmann, C. M. Morales, C. R. Landis, F.Weinhold. (Theoretical Chemistry Institute, University of Wisconsin, Madison, WI, 2013); http://nbo6.chem.wisc.edu/.

[84] F. Weinhold, in Encyclopedia of Computational Chemistry; P. v. R. Schleyer, Ed.; John Wiley \& Sons: Chichester, U.K., 1998; Vol. 3, pp 1792-1811.

[85] F. Weinhold, C. R. Landis, Valency and Bonding: A Natural Bond Orbital Donor-Acceptor Perspective; University Press: Cambridge, U.K., 2003.

[86] D.J. Grant, M.H. Matus, J.R. Switzer, D.A. Dixon, J.S. Francisco, K.O. Christe, Bond Dissociation Energies in Second-Row Compounds, J. Phys. Chem. A, 112 (2008) 3145-3156.

[87] P. Chini, G. Ciani, L. Garlaschelli, M. Manassero, S. Martinengo, A. Sironi, F. Canziani, New tetrahedral cluster compounds of iridium. Synthesis of the anions $\left[\mathrm{Ir}_{4}(\mathrm{CO})_{11} \mathrm{X}\right]^{-}(\mathrm{X}=\mathrm{Cl}, \mathrm{Br}, \mathrm{I}, \mathrm{CN}, \mathrm{SCN})$ and $\mathrm{x}-\mathrm{ray}$ structure of $\left[\mathrm{PPh}_{4}\right]\left[\mathrm{Ir}_{4}(\mathrm{CO})_{11} \mathrm{Br}\right]$, J. Organomet. Chem., 152 (1978) C35-C38.

[88] N. de Silva, A. Solovyov, A. Katz, Patterned metal polyhedra using calixarenes as organizational scaffolds: $\mathrm{Ir}_{4}$-based cluster assemblies, Dalton Trans. 39 (2010) 2194-2197.

[89] J. Lieto, J.J. Rafalko, B.C. Gates, Polymer-bound phosphine-substituted tetrairidium carbonyl clusters: Catalysts for olefin hydrogenation, J. Catal., 62 (1980) 149-156.

[ 90 ] A.G. Orpen, N.G. Connelly, Structural systematics: the role of P-A .sigma.* orbitals in metal-phosphorus .pi.-bonding in redox-related pairs of $\mathrm{M}-\mathrm{PA}_{3}$ complexes $(\mathrm{A}=\mathrm{R}, \mathrm{Ar}, \mathrm{OR} ; \mathrm{R}=\mathrm{alkyl})$, Organometallics, 9 (1990) 1206-1210.

[91] G.F. Stuntz, J.R. Shapley, Intracluster ligand mobility. 4. Solution structures and dynamics of $\mathrm{Ir}_{4}(\mathrm{CO})_{12-\mathrm{x}}\left(\mathrm{PPh}_{2} \mathrm{Me}\right)_{\mathrm{x}}(\mathrm{x}=1-4)$, J. Am. Chem. Soc., 99 (1977) 607-609.

[92] F.A. Cotton, A Solution to the Structural Dilemma of $\mathrm{Co}_{4}(\mathrm{CO})_{12}$ Based on Valence Tautomerism and Steric Nonrigidity, Inorg. Chem., 5 (1966) 1083-1085.

[93] F.A. Cotton, L. Kruczynski, B.L. Shapiro, L.F. Johnson, Direct evidence from carbon-13 nuclear magnetic resonance for intramolecular scrambling of carbonyl groups in a metal atom cluster carbonyl, tetrarhodium dodecacarbonyl, J. Am. Chem. Soc., 94 (1972) 6191-6193.

[94] F.S. Livotto, P.R. Raithby, M.D. Vargas, Synthesis and structural characterization of the phosphido cluster $\left[\mathrm{Ir}_{4} \mathrm{H}(\mathrm{CO})_{10}\left(\mu-\mathrm{PPh}_{2}\right)\right]$ and its facile carbonyl substitution reactions with a series of phosphines and phosphites, $\mathrm{J}$. Chem. Soc., Dalton Trans., (1993) 1797-1803.

95 M.J. Davis, R. Roulet, Fluxional behaviour of $\left[\operatorname{Ir}_{4}(\mathrm{CO})_{11} \mathrm{H}\right]^{-}$in solution, Inorg. Chim. Acta, 197 (1992) 15-20.

[96] D.C. Sonnenberger, J.D. Atwood, Labilization of carbon monoxide dissociation from metal clusters: an investigation of the substitution reactions of monosubstituted derivatives of tetrairidium, $\operatorname{Ir}_{4}(\mathrm{CO})_{11} \mathrm{~L}\left(\mathrm{~L}=\mathrm{PBu}_{3}\right.$, $\mathrm{PPh}_{3}, \mathrm{AsPh}_{3}$, and $\left.\mathrm{P}(\mathrm{OPh})_{3}\right)$, J. Am. Chem. Soc., 104 (1982) 2113-2116.

[97] J.D. Atwood, M.J. Wovkulich, D.C. Sonnenberger, Ligand effects on organometallic substitution reactions, Acc. Chem. Res., 16 (1983) 350-355.

[98] M.J. Wovkulich, J.L. Atwood, L. Canada, J.D. Atwood, A crystallographic determination of the influence of the trans ligand on the bonding of triphenylphosphine. Crystal and molecular structures of $\mathrm{Cr}(\mathrm{CO})_{4}\left(\mathrm{PPh}_{3}\right) \mathrm{L}$ $\left(\mathrm{L}=\mathrm{PBu}_{3}, \mathrm{P}(\mathrm{OMe})_{3}\right.$, and $\left.\mathrm{P}(\mathrm{OPh})_{3}\right)$, Organometallics, 4 (1985) 867-871. 


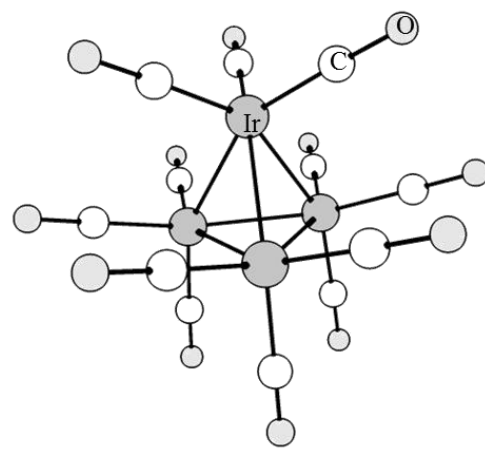

a

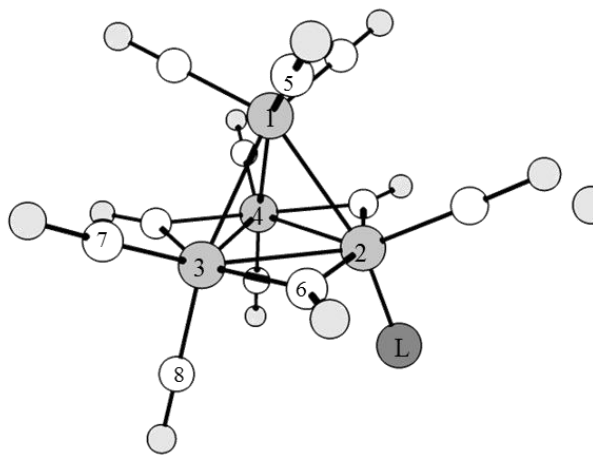

b

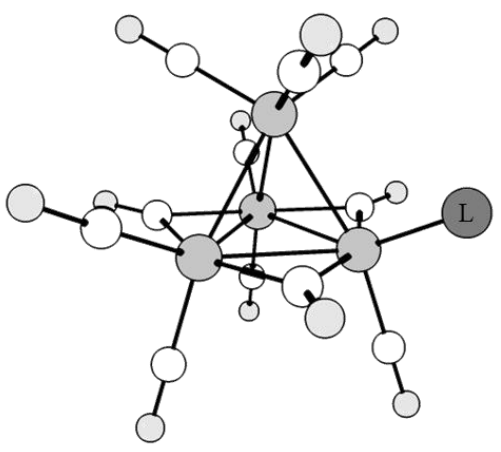

C

Figure 1: Isomers of $\operatorname{Ir}_{4}(\mathrm{CO})_{12}$ and $\operatorname{Ir}_{4}(\mathrm{CO})_{11} \mathrm{~L}$. In $\mathrm{T}_{\mathrm{d}}$ structure a, all carbonyls are terminal. In $\mathrm{C}_{3 \mathrm{v}}$ structure $\mathbf{b}, \operatorname{Ir}(1)$ is apical iridium, and $\operatorname{Ir}(2), \operatorname{Ir}(3)$, and $\operatorname{Ir}(4)$ are basal iridiums that build up the basal plane. $\mathrm{C}(5), \mathrm{C}(6), \mathrm{C}(7)$, and $\mathrm{C}(8)$ are apical, bridging, equatorial, and axial carbon, respectively. $\mathrm{L}$ is the ligand that substitutes for axial carbonyl in $\mathbf{b}$ and for equatorial carbonyl in c. 


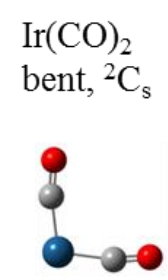

0.0

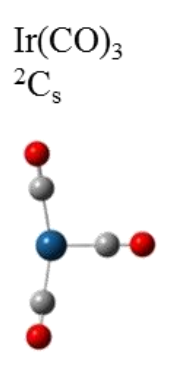

$1 \mathrm{a}$

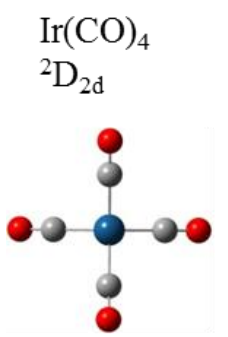

$1 \mathrm{~g}$

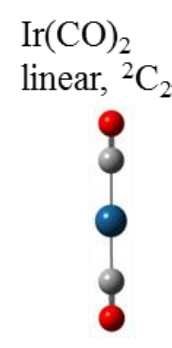

9.9

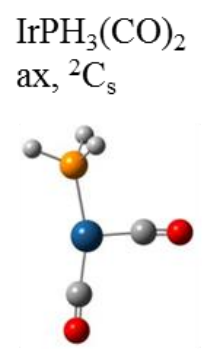

1b 0.0

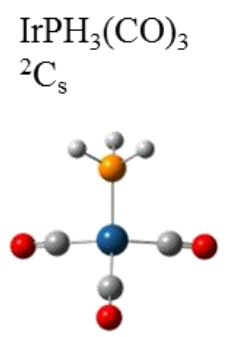

$1 \mathrm{~h}$

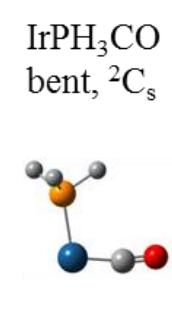

0.0

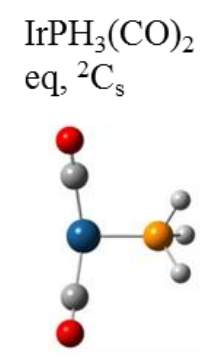

1c 2.2

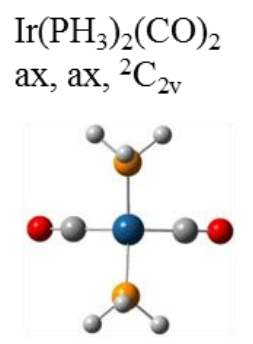

$1 i 0.0$

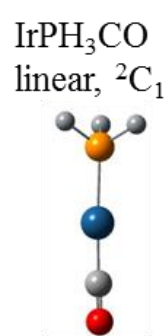

6.8

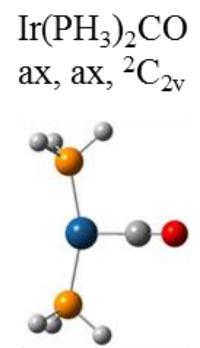

1d 0.0

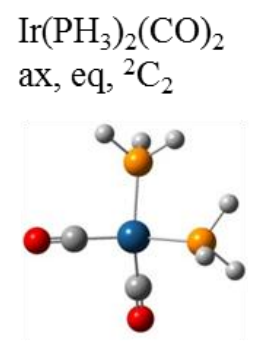

1j 1.4

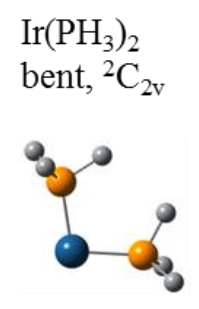

0.0

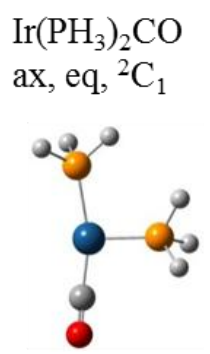

1e 1.3

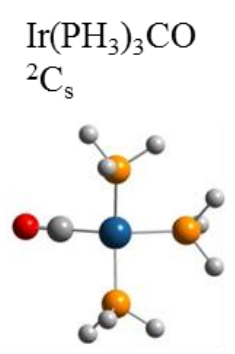

$1 k$

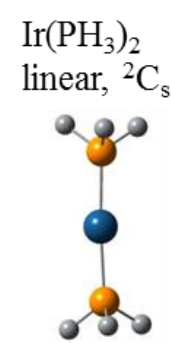

5.9

$$
\operatorname{Ir}\left(\mathrm{PH}_{3}\right)_{3}
$$$$
{ }^{2} \mathrm{C}_{\mathrm{s}}
$$

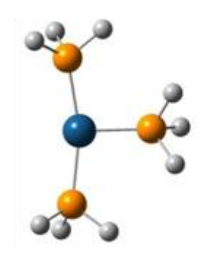

1f

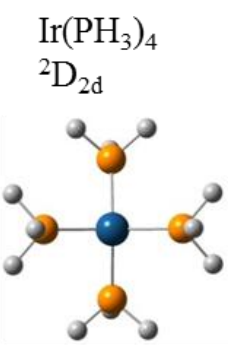

11

Figure 1: Optimized geometries of low-lying $\operatorname{Ir}\left(\mathrm{PH}_{3}\right)_{y}(\mathrm{CO})_{\mathrm{z}}$ isomers and their symmetries. Only doublet states are shown. Relative energies are in $\mathrm{kcal} / \mathrm{mol}$. 


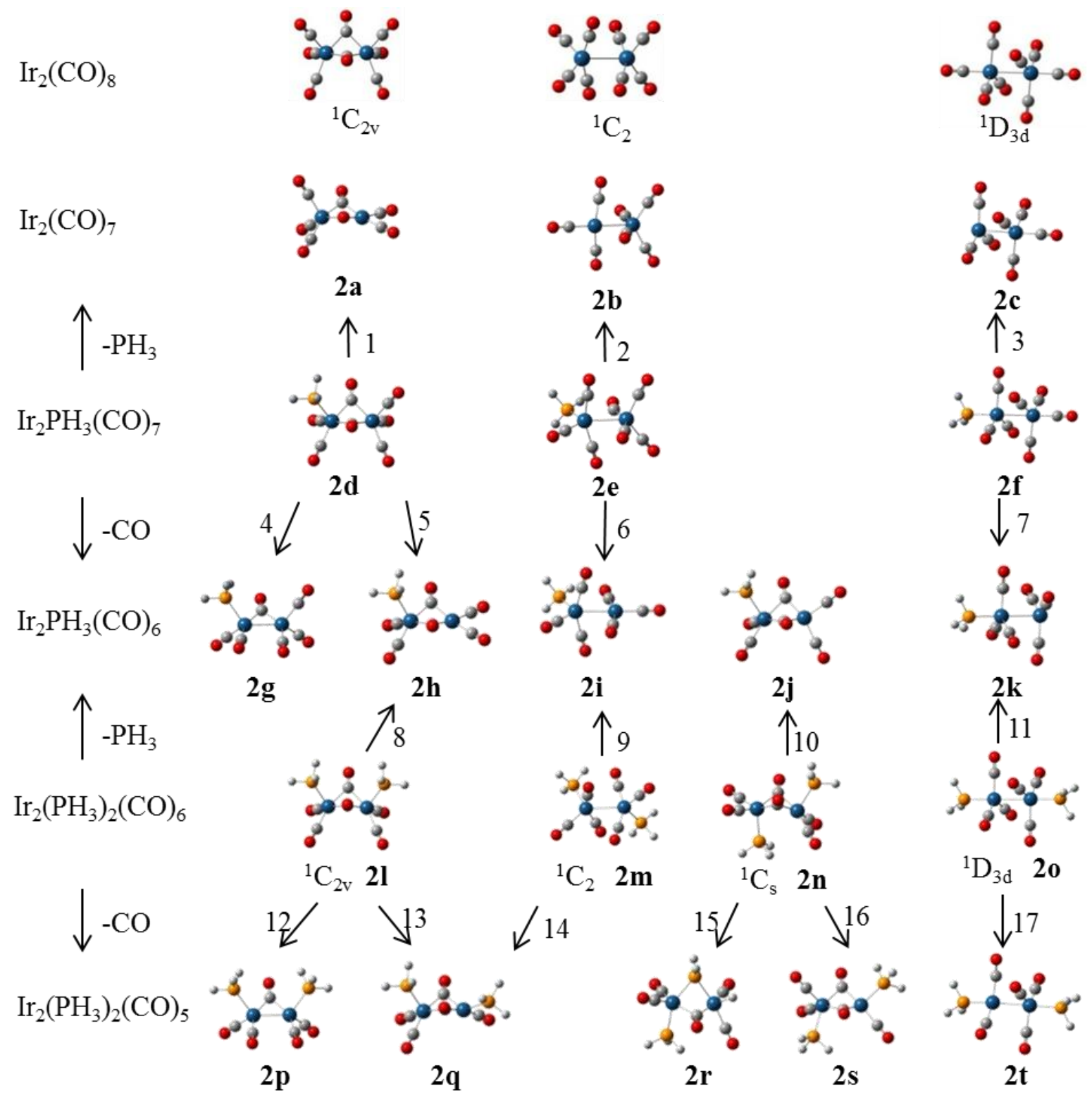

Figure 1: Optimized geometries of low-lying $\operatorname{Ir}_{2}\left(\mathrm{PH}_{3}\right)_{\mathrm{y}}(\mathrm{CO})_{\mathrm{z}}$ isomers. 
$\operatorname{Ir}_{4}(\mathrm{CO})_{11}$

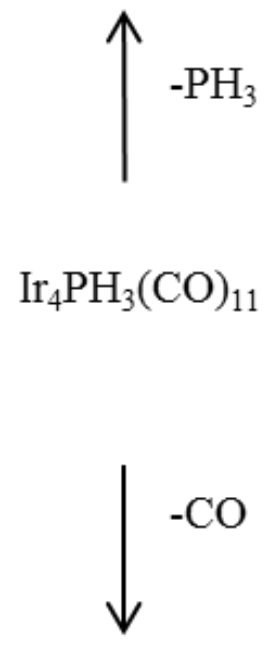

$\mathrm{Ir}_{4} \mathrm{PH}_{3}(\mathrm{CO})_{10}$

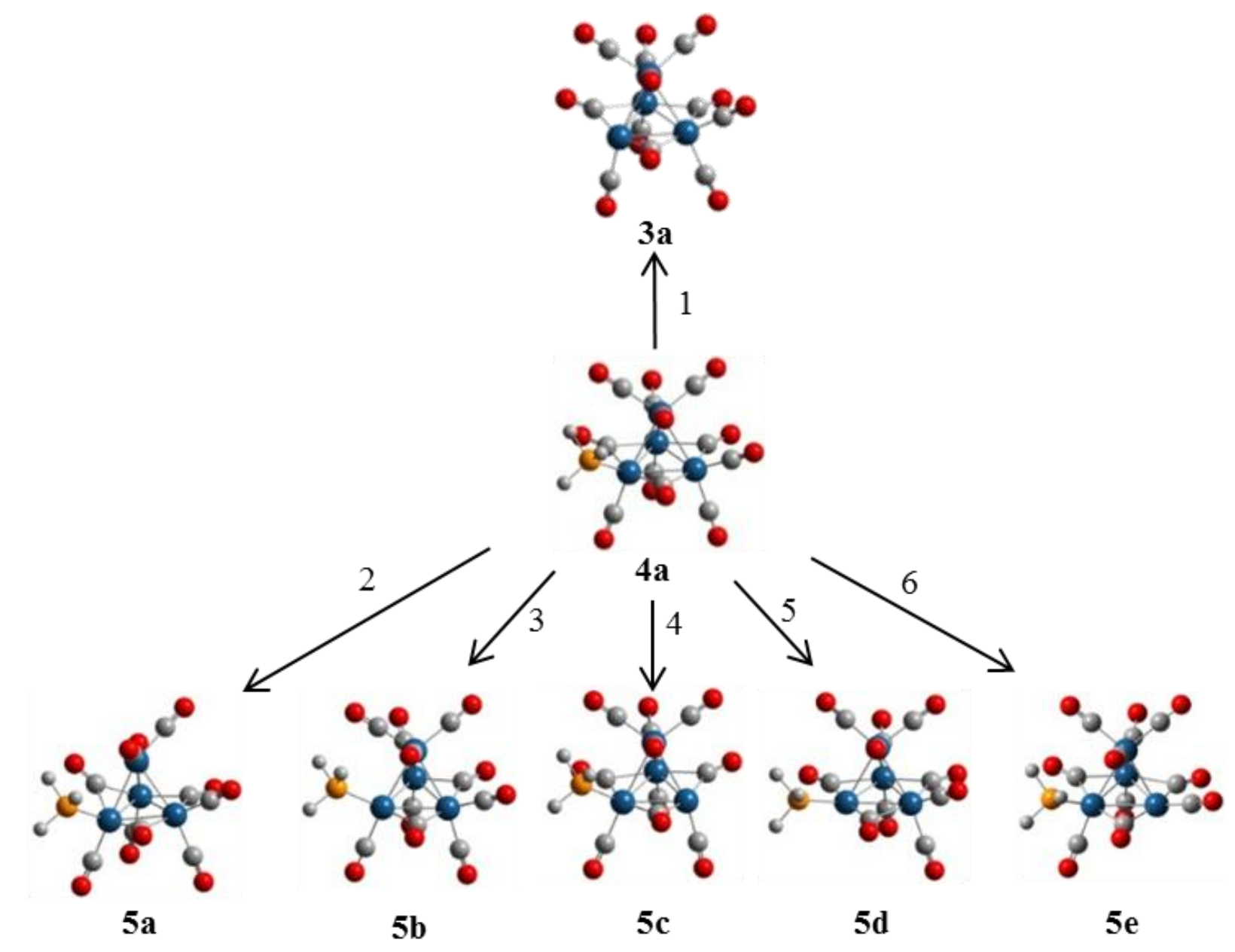

Figure 1: Optimized geometries of low-lying $\operatorname{Ir}_{4}\left(\mathrm{PH}_{3}\right)_{y}(\mathrm{CO})_{z}$ isomers 


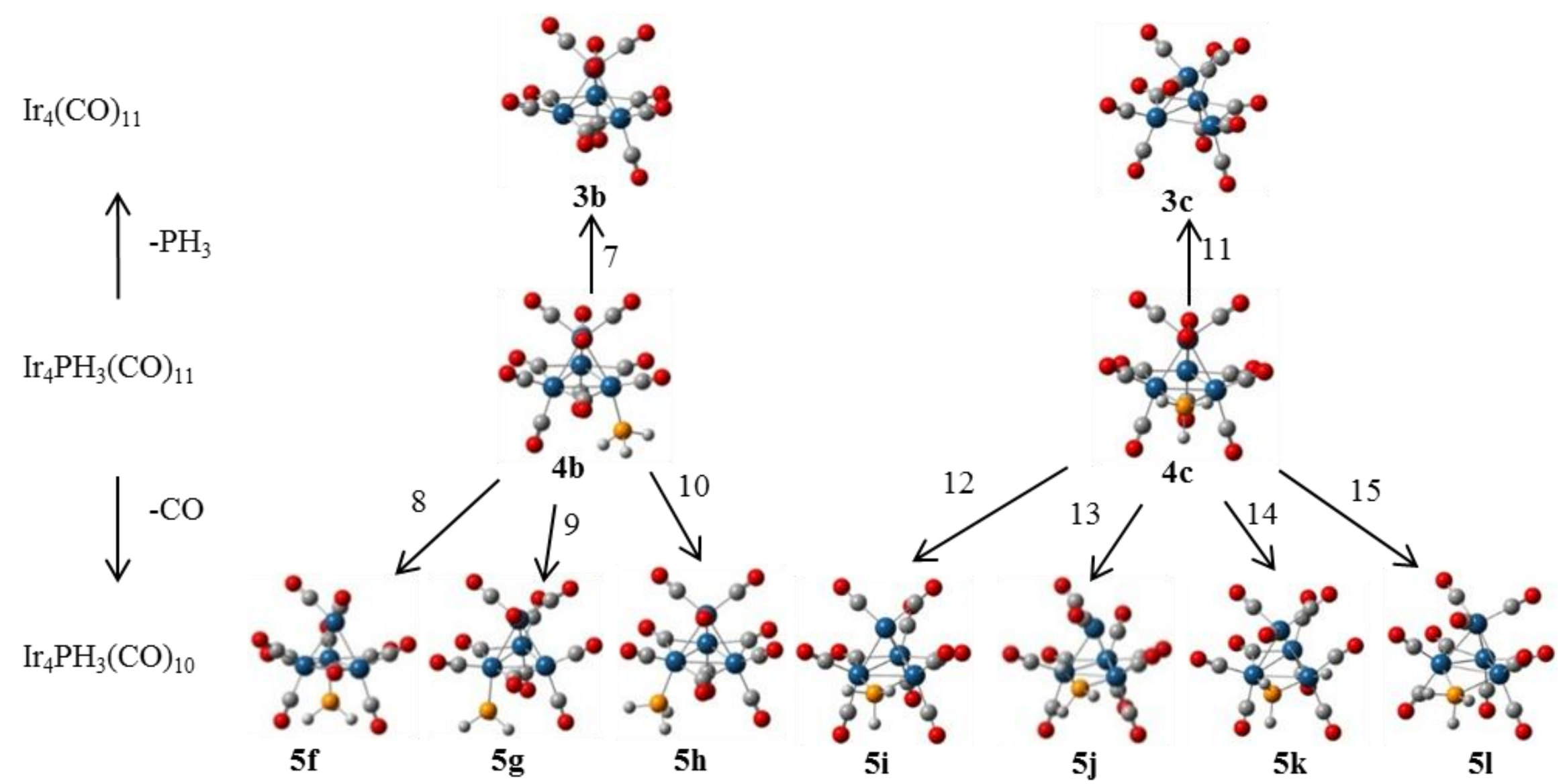

Figure 4 (continued): Optimized geometries of low-lying $\operatorname{Ir} 4(\mathrm{PH} 3) \mathrm{y}(\mathrm{CO}) \mathrm{z}$ isomers. 


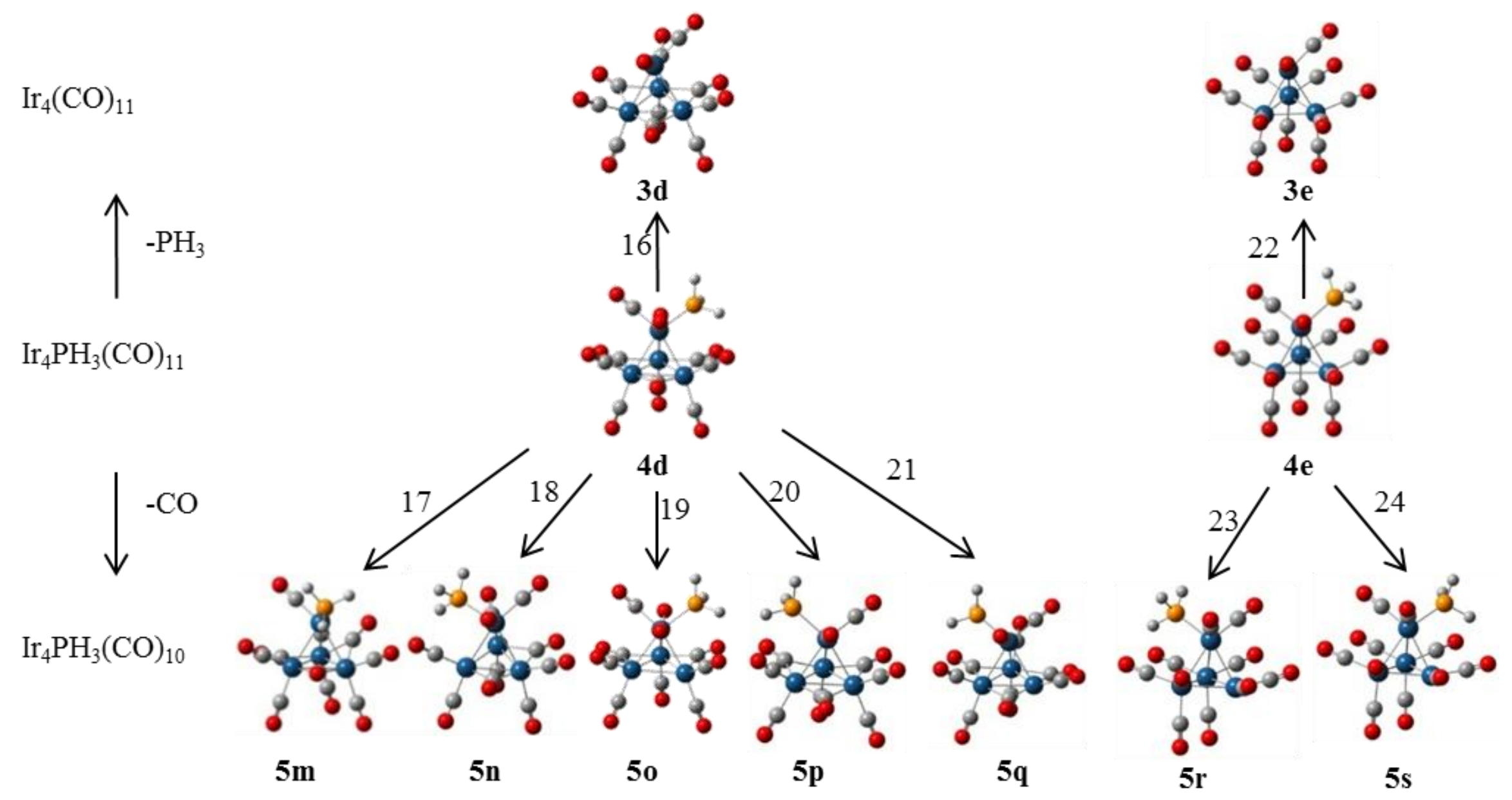

Figure 4 (continued): Optimized geometries of low-lying $\operatorname{Ir} 4(\mathrm{PH} 3) \mathrm{y}(\mathrm{CO}) \mathrm{z}$ isomers. 
$\mathrm{Ir}_{4} \mathrm{PH}_{3}(\mathrm{CO})_{10}$

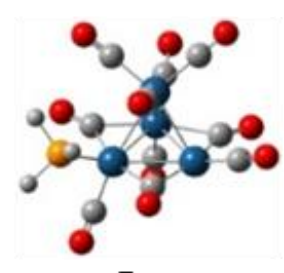

$5 e$

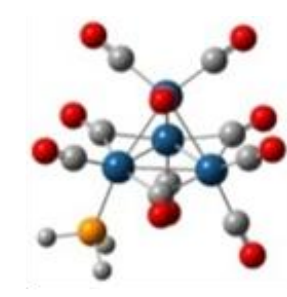

5 h

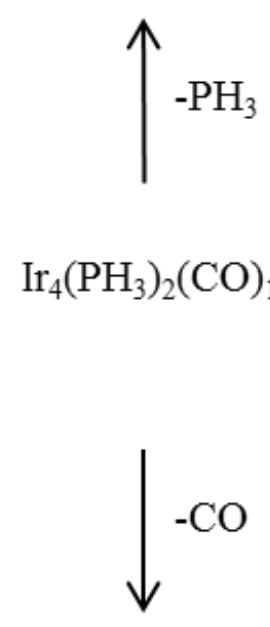

$\mathrm{Ir}_{4}\left(\mathrm{PH}_{3}\right)_{2}(\mathrm{CO})_{9}$
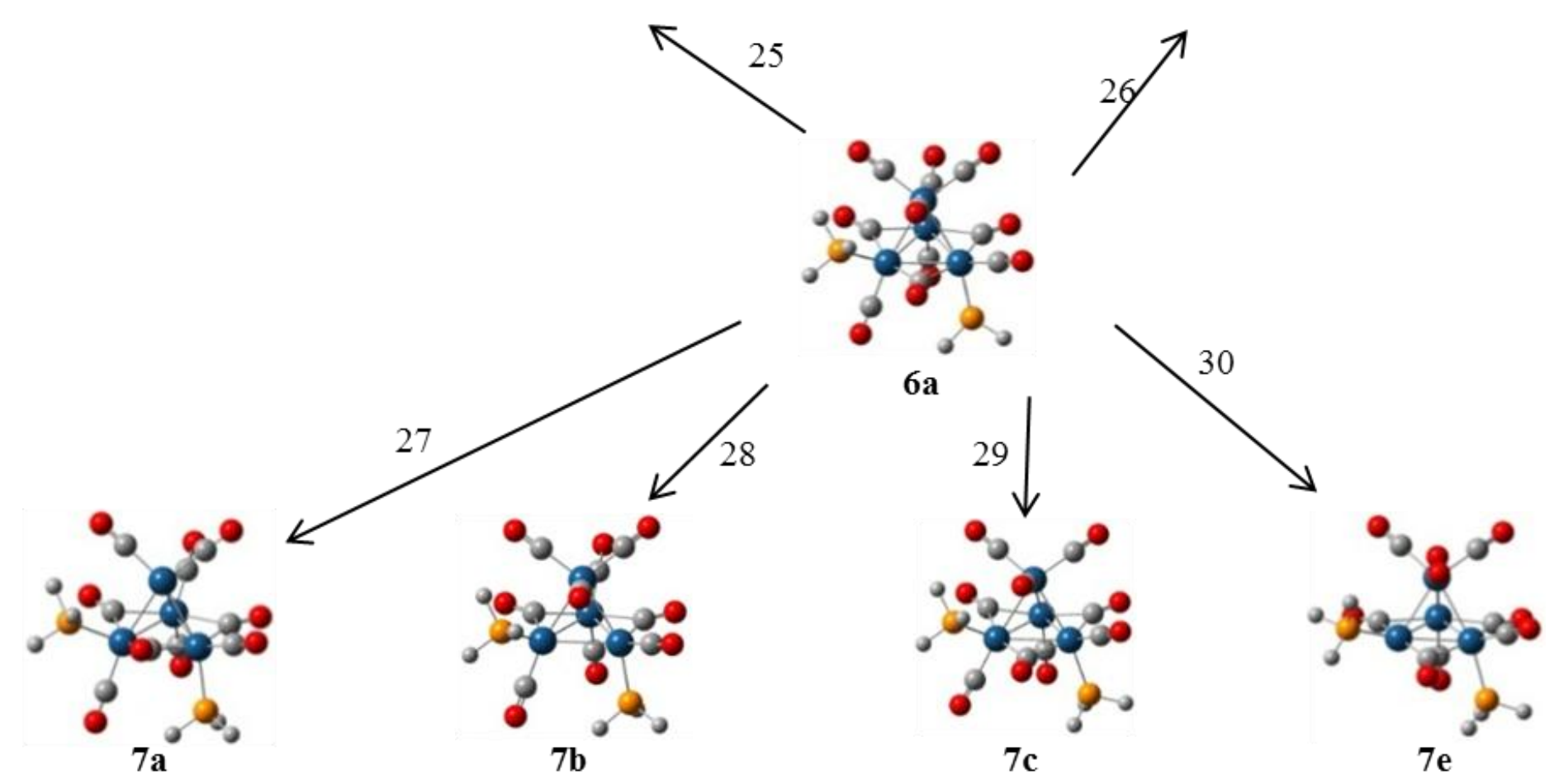

Figure 4 (continued): Optimized geometries of low-lying $\operatorname{Ir} 4(\mathrm{PH} 3) \mathrm{y}(\mathrm{CO}) \mathrm{z}$ isomers. 
$\mathrm{Ir}_{4} \mathrm{PH}_{3}(\mathrm{CO})_{10}$
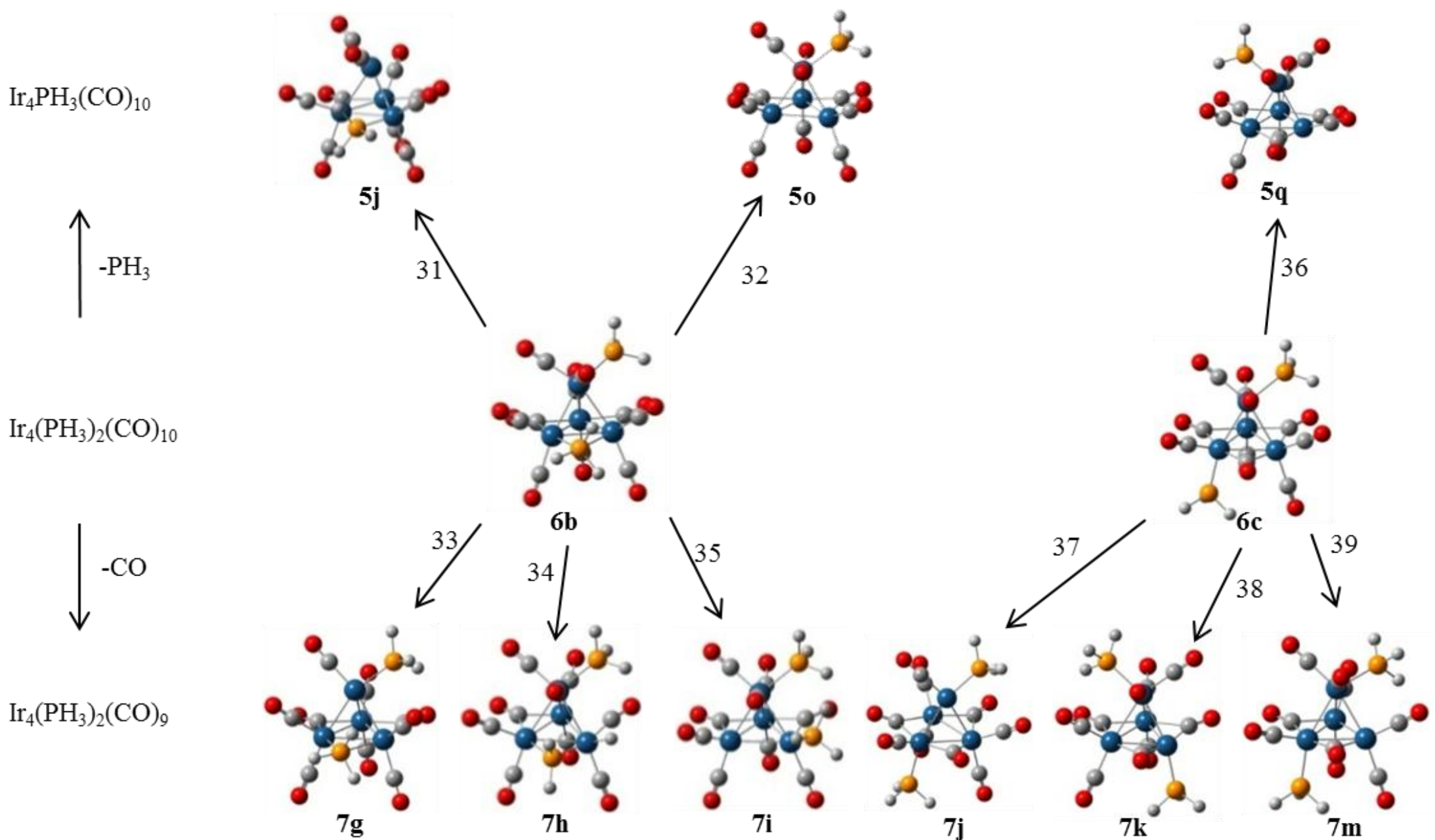

Figure 4 (continued): Optimized geometries of low-lying $\operatorname{Ir} 4(\mathrm{PH} 3) \mathrm{y}(\mathrm{CO}) \mathrm{z}$ isomers. 
$\mathrm{Ir}_{4} \mathrm{PH}_{3}(\mathrm{CO})_{10}$

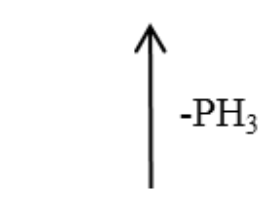

$\mathrm{Ir}_{4}\left(\mathrm{PH}_{3}\right)_{2}(\mathrm{CO})_{10}$

$\downarrow^{-\mathrm{CO}}$

$\mathrm{Ir}_{4}\left(\mathrm{PH}_{3}\right)_{2}(\mathrm{CO})_{9}$

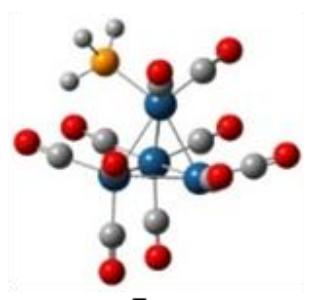

$5 r$

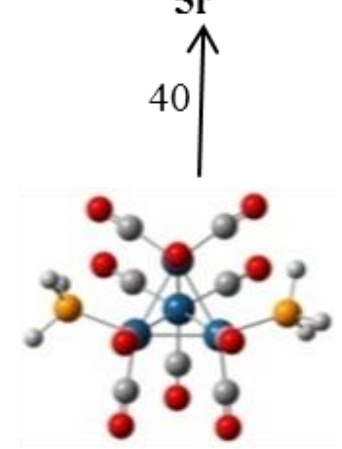

6d

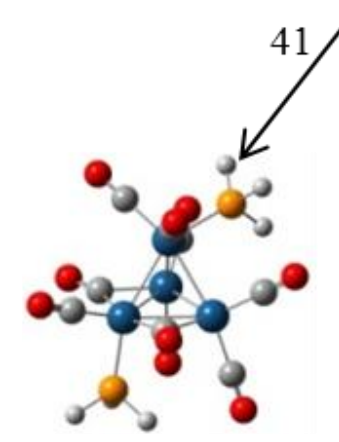

$7 \mathbf{m}$

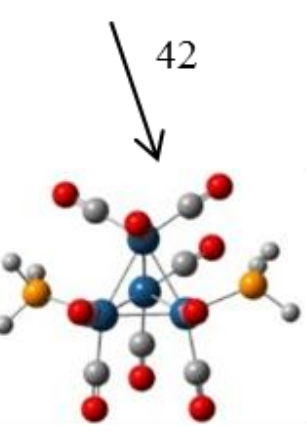

$7 n$

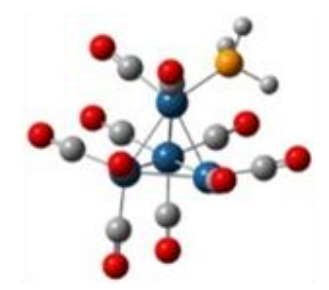

5s
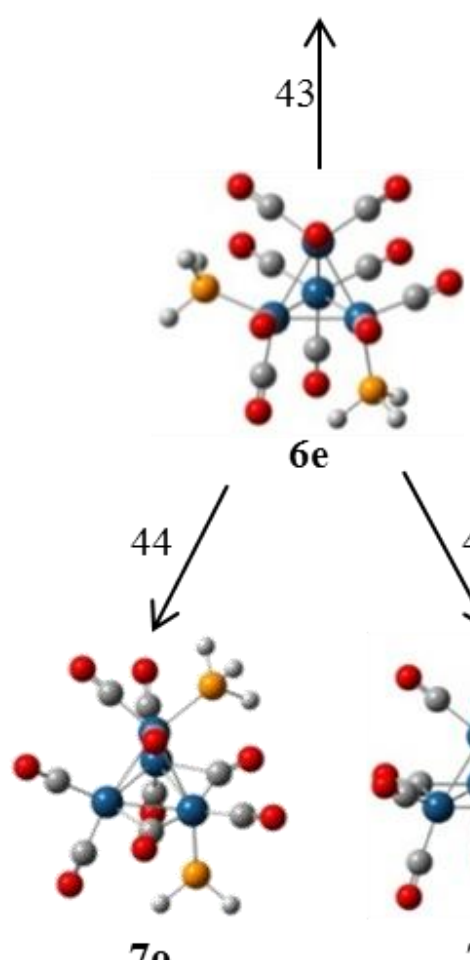

7o

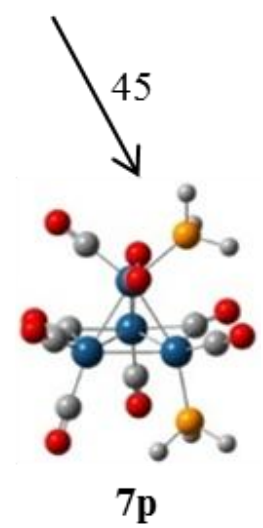

Figure 4 (continued): Optimized geometries of low-lying $\operatorname{Ir} 4(\mathrm{PH} 3) \mathrm{y}(\mathrm{CO}) \mathrm{z}$ isomers. 
$\mathrm{Ir}_{4}\left(\mathrm{PH}_{3}\right)_{2}(\mathrm{CO})_{9}$
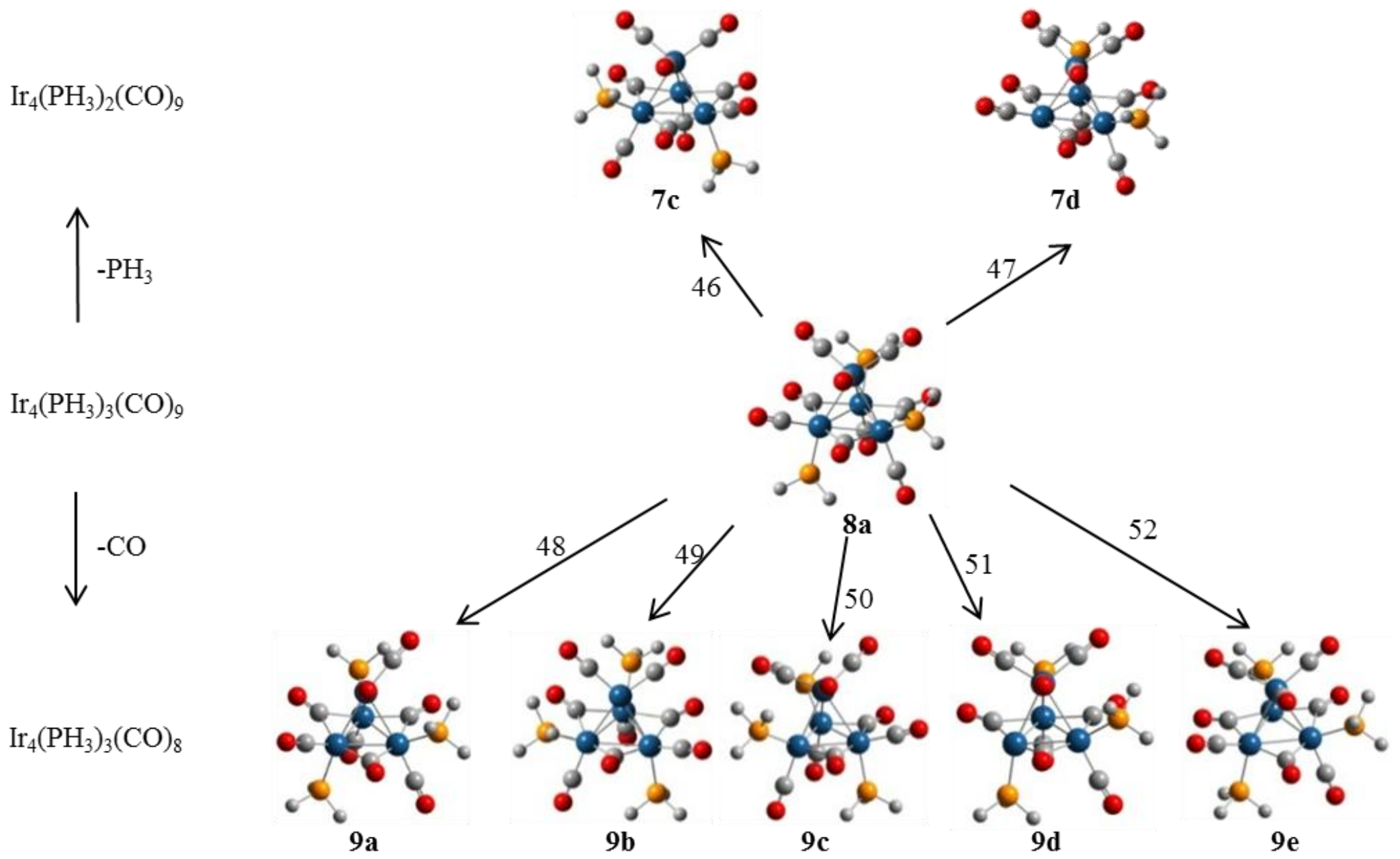

Figure 4 (continued): Optimized geometries of low-lying $\operatorname{Ir} 4(\mathrm{PH} 3) \mathrm{y}(\mathrm{CO}) \mathrm{z}$ isomers. 
$\mathrm{Ir}_{4}\left(\mathrm{PH}_{3}\right)_{2}(\mathrm{CO})_{9}$

$\uparrow-\mathrm{PH}_{3}$

$\mathrm{Ir}_{4}\left(\mathrm{PH}_{3}\right)_{3}(\mathrm{CO})_{9}$

$\downarrow-\mathrm{CO}$

$\mathrm{Ir}_{4}\left(\mathrm{PH}_{3}\right)_{3}(\mathrm{CO})_{8}$

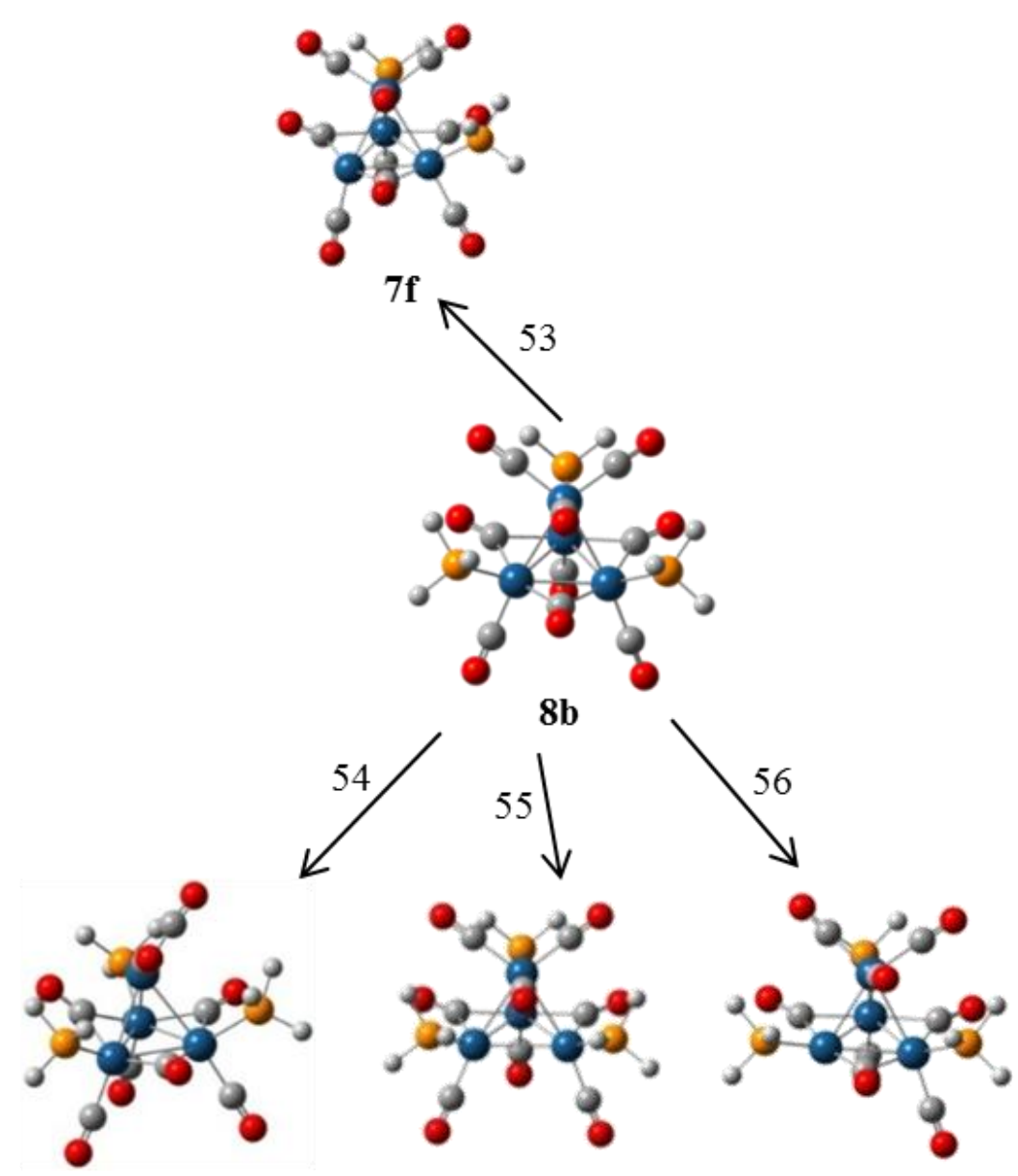

$9 h$

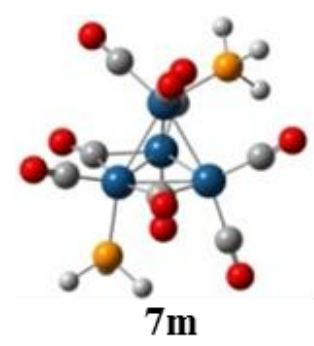

$62 \uparrow$

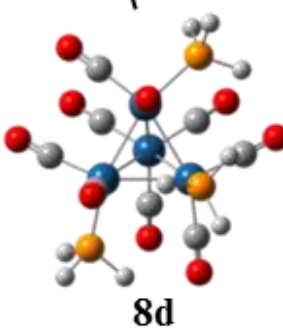

$63 \downarrow$

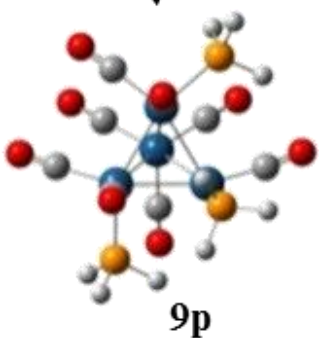

Figure 4 (continued): Optimized geometries of low-lying $\operatorname{Ir} 4(\mathrm{PH} 3) \mathrm{y}(\mathrm{CO}) \mathrm{z}$ isomers. 
$\mathrm{Ir}_{4}\left(\mathrm{PH}_{3}\right)_{2}(\mathrm{CO})_{9}$

个 $-\mathrm{PH}_{3}$

$\mathrm{Ir}_{4}\left(\mathrm{PH}_{3}\right)_{3}(\mathrm{CO})_{9}$

$\downarrow^{-\mathrm{CO}}$

$\mathrm{Ir}_{4}\left(\mathrm{PH}_{3}\right)_{3}(\mathrm{CO})_{8}$
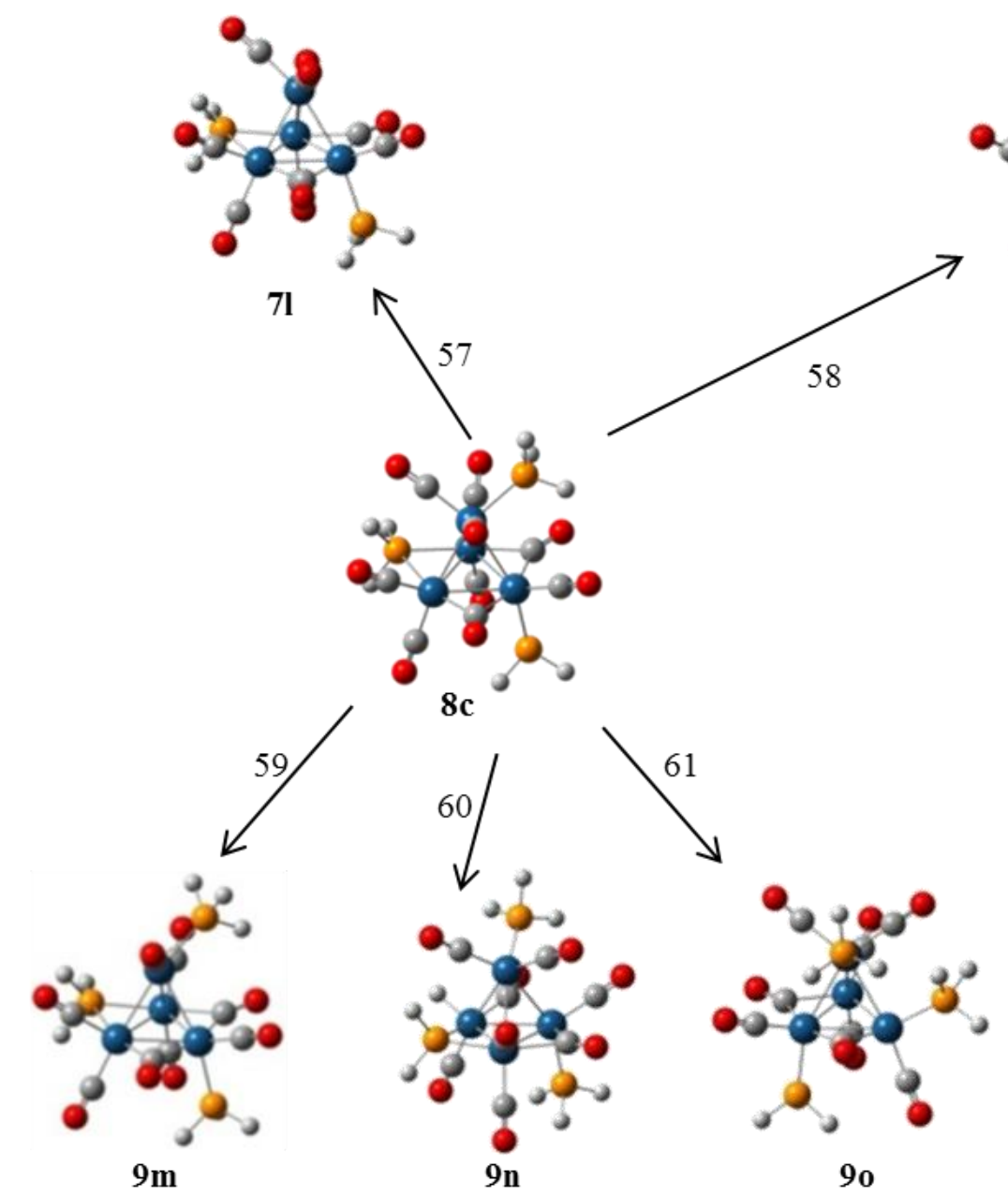

90

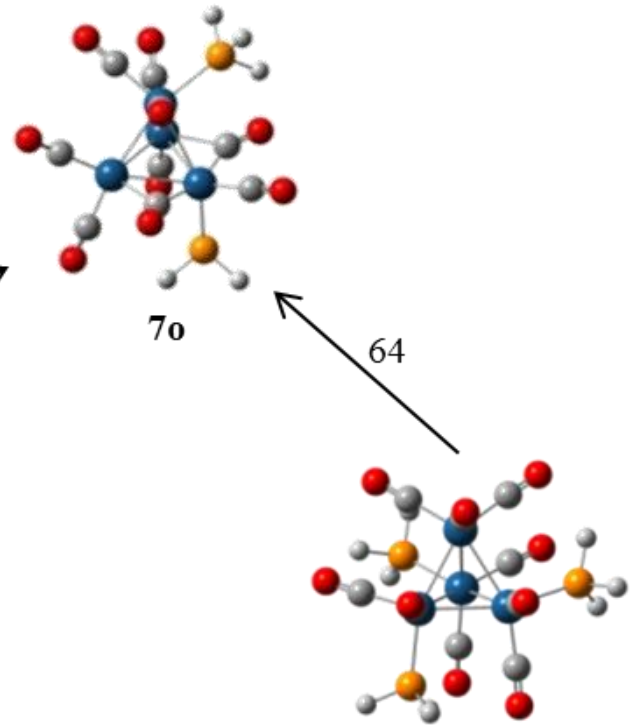

8 e

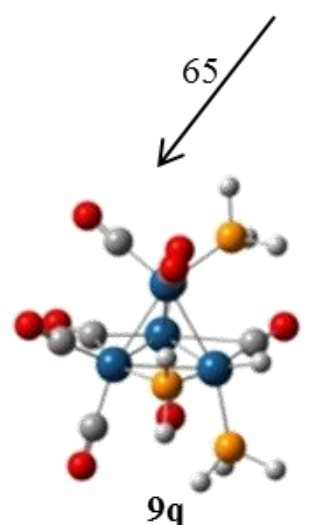

$9 q^{3}$

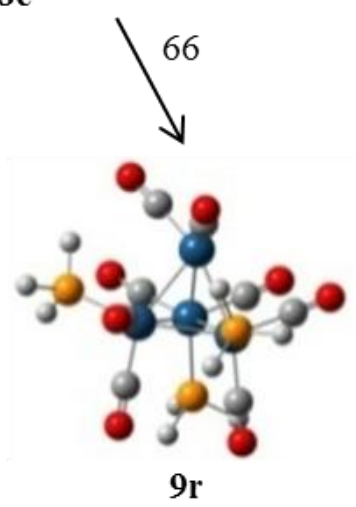

Figure 4 (continued): Optimized geometries of low-lying $\operatorname{Ir} 4(\mathrm{PH} 3) \mathrm{y}(\mathrm{CO}) \mathrm{z}$ isomers. 


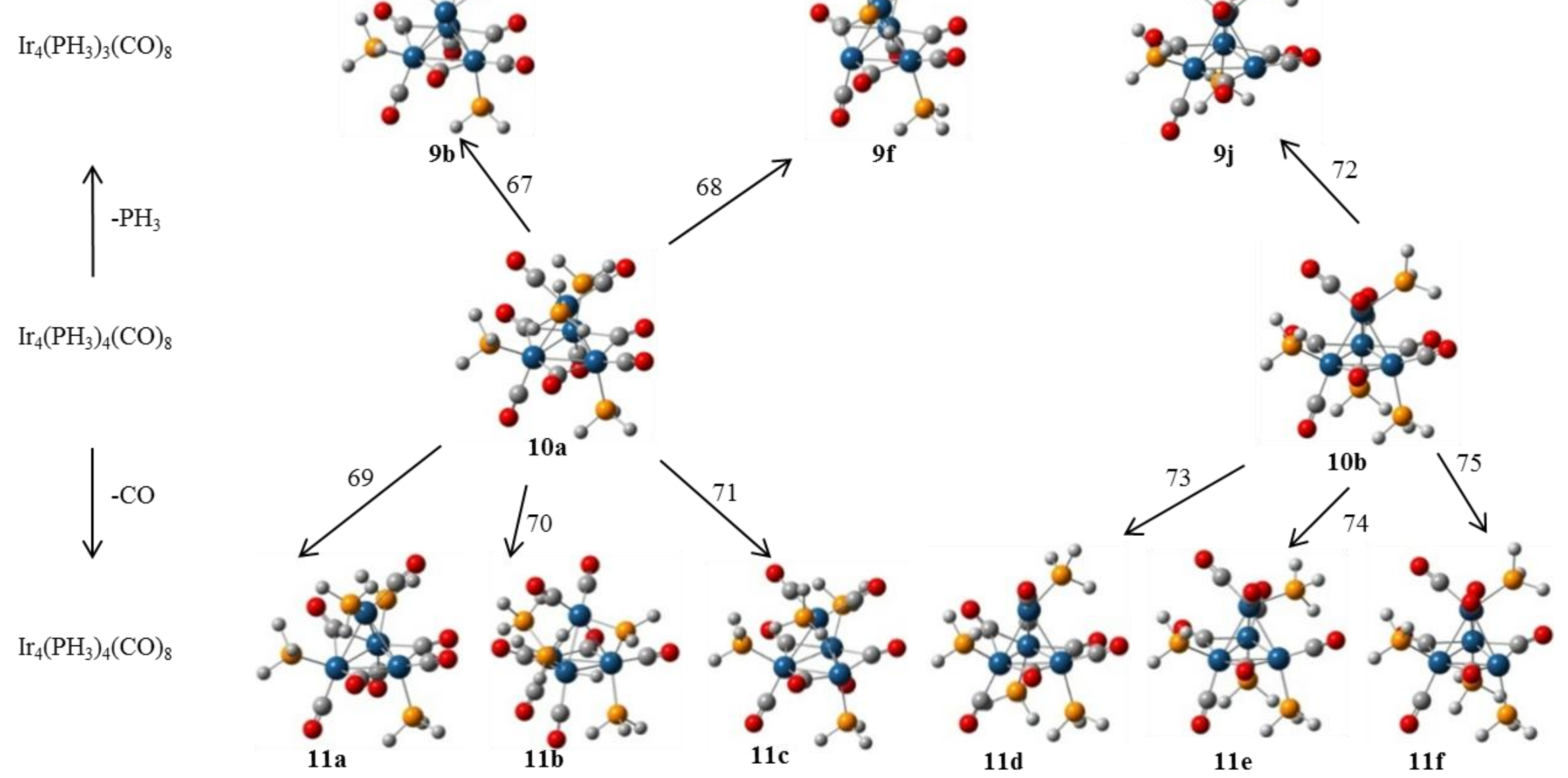

Figure 4 (continued): Optimized geometries of low-lying $\operatorname{Ir} 4(\mathrm{PH} 3) \mathrm{y}(\mathrm{CO}) \mathrm{z}$ isomers. 
$\mathrm{Ir}_{4}\left(\mathrm{PH}_{3}\right)_{3}(\mathrm{CO})_{8}$
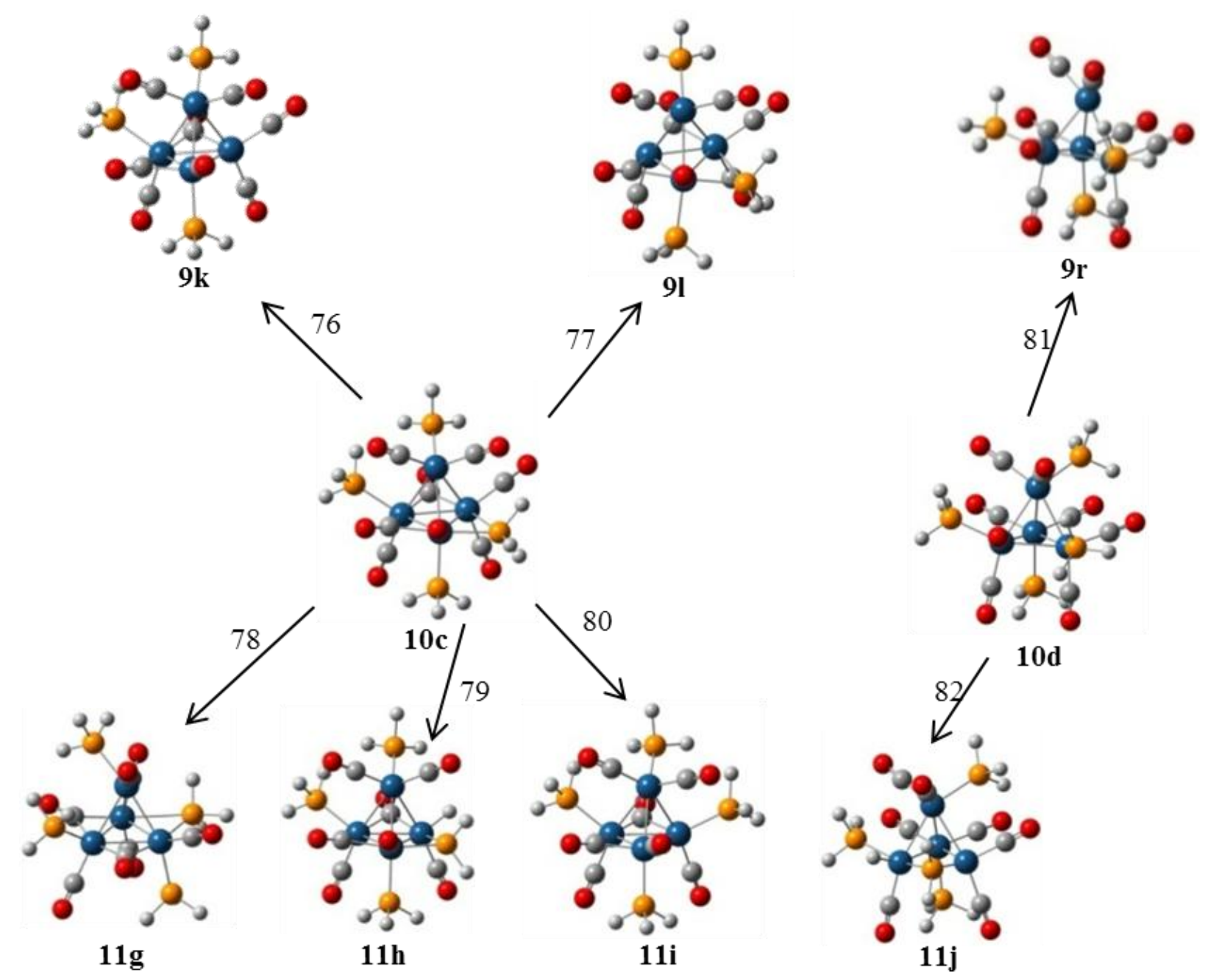

Figure 2: Optimized geometries of low-lying $\operatorname{Ir}_{4}\left(\mathrm{PH}_{3}\right)_{y}(\mathrm{CO})_{\mathrm{z}}$ isomers 
(a)

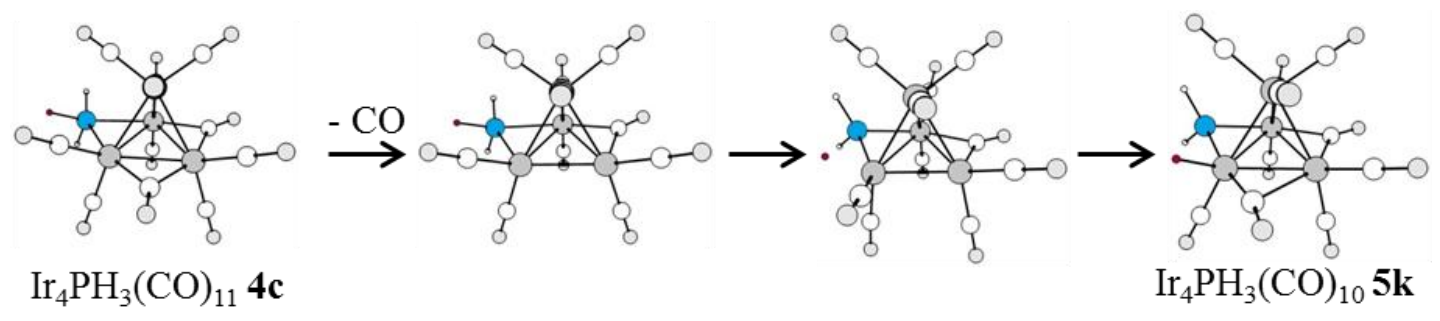

(b)
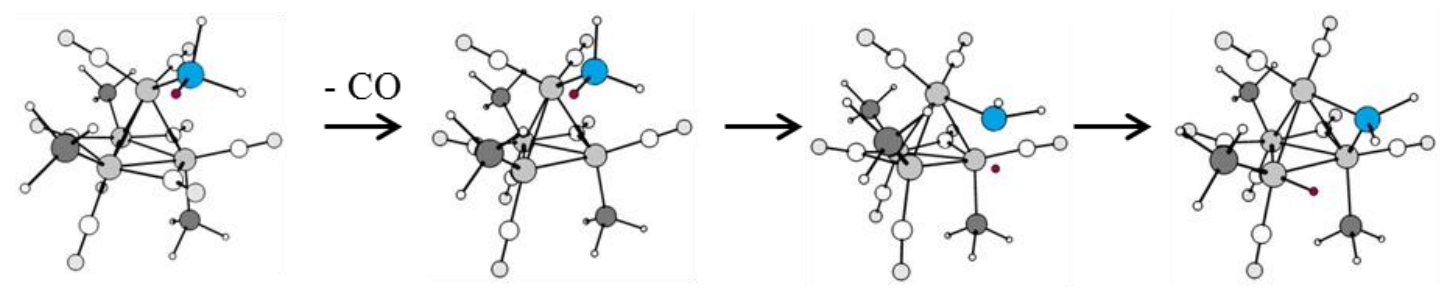

$\mathrm{Ir}_{4}\left(\mathrm{PH}_{3}\right)_{4}(\mathrm{CO})_{8}$ 10a

(c)
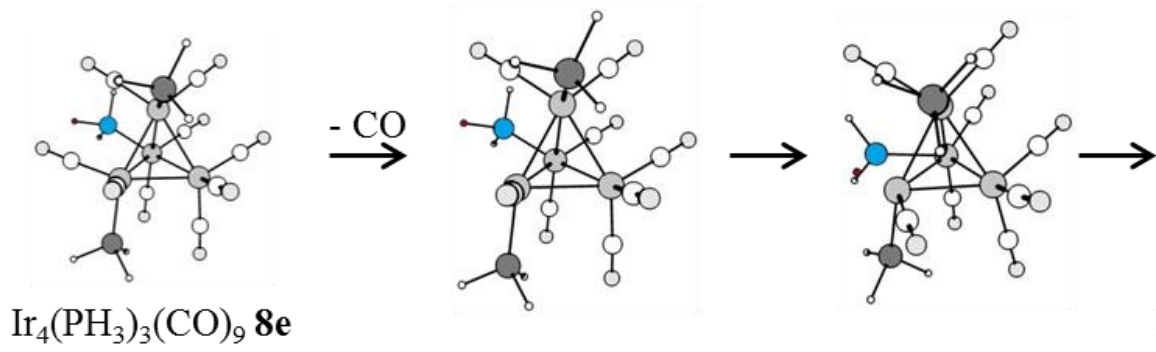

$\mathrm{Ir}_{4}\left(\mathrm{PH}_{3}\right)_{4}(\mathrm{CO})_{7} \mathbf{1 1 b}$

Figure 1: Three types of $\mathrm{H}$ atom transfer reactions that occur on $\mathrm{CO}$ dissociation. The $\mathrm{H}$ and $\mathrm{P}$ atoms involved in the transfer reactions are marked in red and blue, respectively. The isomers in mid two columns are for description, not transition states or intermediates. 
(a)

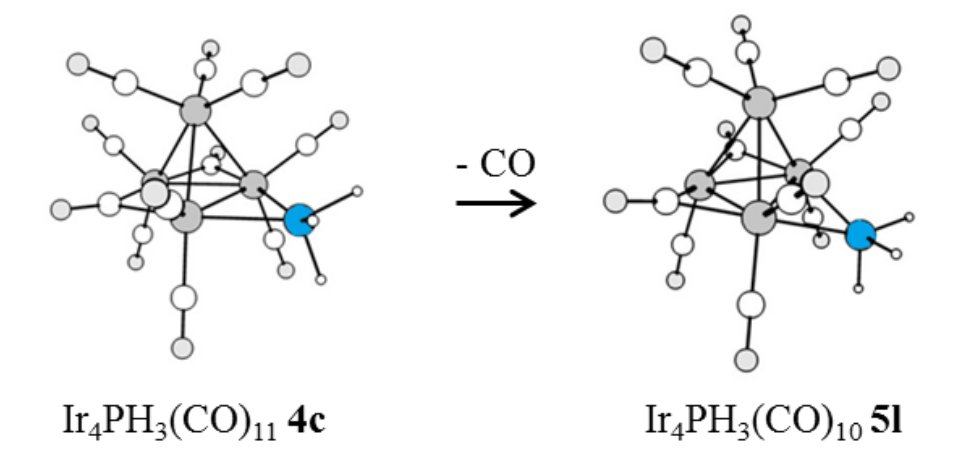

(b)
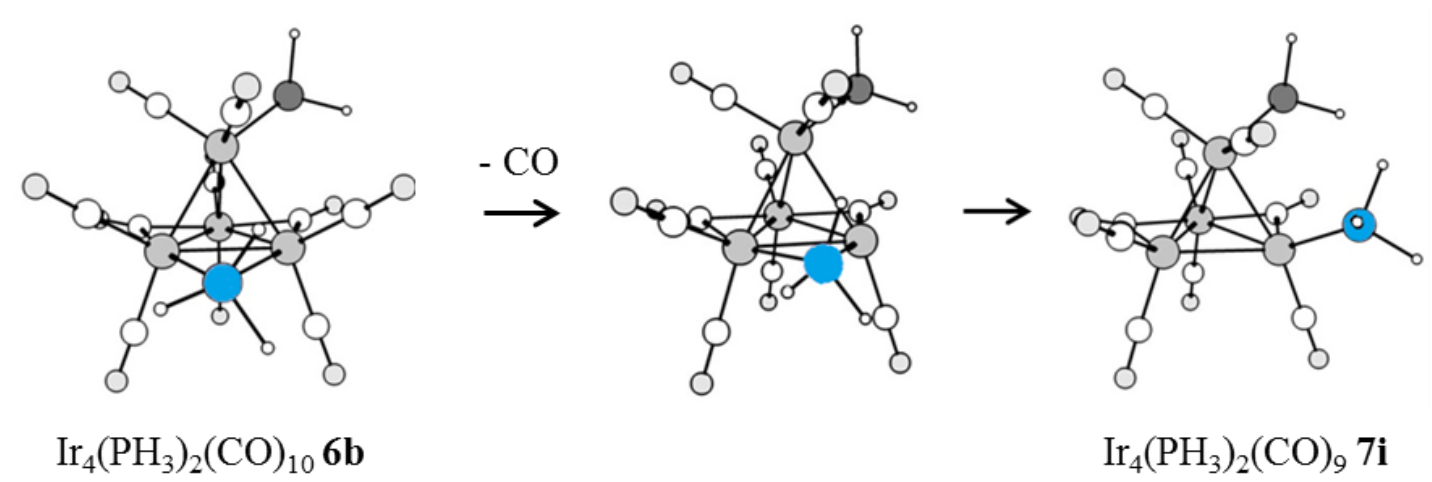

Figure 1: (a) A Bridging PH3 Maintained Process. (b) A Transformation from Bridging $\mathrm{PH} 3$ to Equatorial One. The $\mathrm{P}$ atoms involved in the transformation are marked in blue. 
Table 1. Relative Energies of $\operatorname{Ir}\left(\mathrm{PH}_{3}\right)_{\mathrm{y}}(\mathrm{CO})_{\mathrm{z}}$ Isomers in kcal/mol, predicted by various electronic structure methods. ${ }^{\text {a }}$

\begin{tabular}{|c|c|c|c|c|c|c|c|c|c|c|c|c|c|}
\hline Molecule & Symmetry & BP86 & $\mathrm{PBE}$ & PW91 & B3LYP & $\begin{array}{l}\text { CAM- } \\
\text { B3LYP }\end{array}$ & B97-D & B98 & $\omega B 97 X$ & $\begin{array}{c}\omega B 97 X \\
-D\end{array}$ & M06 & MP2 & $\operatorname{CCSD}(\mathrm{T})$ \\
\hline${ }^{4} \mathrm{IrCO}$ & $\mathrm{C}_{\mathrm{s}}$ & 39.7 & 39.7 & 39.7 & 37.4 & 35.1 & 46.6 & 40.4 & 42.3 & 41.5 & 44.9 & 44.9 & 36.3 \\
\hline${ }^{2} \operatorname{Ir}(\mathrm{CO})_{2}$ & $\mathrm{C}_{2 \mathrm{v}}$ linear & -1.5 & -0.1 & -0.5 & -8.7 & -10.7 & -2.3 & -8.3 & -11.3 & -8.9 & -6.3 & 15.8 & 9.9 \\
\hline${ }^{2} \operatorname{Ir}(\mathrm{CO})_{3}$ & $\mathrm{C}_{\mathrm{s}}$ & 0.0 & 0.0 & 0.0 & 0.0 & 0.0 & 0.0 & 0.0 & 0.0 & 0.0 & 0.0 & 0.0 & 0.0 \\
\hline${ }^{4} \operatorname{Ir}(\mathrm{CO})_{3}$ & $\mathrm{C}_{2 \mathrm{v}}$ & 49.9 & 49.6 & 49.8 & 47.8 & 45.3 & 54.5 & 49.0 & 49.0 & 49.9 & 55.8 & 58.1 & 49.2 \\
\hline${ }^{2} \mathrm{IrPH}_{3}(\mathrm{CO})_{2}$ & $\mathrm{C}_{\mathrm{s}} a x$ & 0.0 & 0.0 & 0.0 & 0.0 & 0.0 & 0.0 & 0.0 & 0.0 & 0.0 & 0.0 & 0.0 & 0.0 \\
\hline${ }^{2} \mathrm{IrPH}_{3}(\mathrm{CO})_{2}$ & $\mathrm{C}_{\mathrm{s}} e q$ & 1.2 & 1.1 & 1.1 & 1.5 & 2.2 & 0.5 & 1.5 & 2.6 & 2.3 & 1.8 & 2.6 & 2.2 \\
\hline${ }^{4} \mathrm{IrPH}_{3}(\mathrm{CO})_{2}$ & $\mathrm{C}_{\mathrm{s}} \operatorname{ax}$ & 53.8 & 53.5 & 53.8 & 51.6 & 49.1 & 58.5 & 52.8 & 52.9 & 53.8 & 60.0 & 62.6 & 52.7 \\
\hline${ }^{4} \mathrm{IrPH}_{3}(\mathrm{CO})_{2}$ & $\mathrm{C}_{\mathrm{s}} e q$ & 48.5 & 48.3 & 48.4 & 45.5 & 43.0 & 52.5 & 46.7 & 46.9 & 47.8 & 53.5 & 107.3 & 94.2 \\
\hline${ }^{2} \mathrm{IrPH}_{3}(\mathrm{CO})_{3}$ & $\mathrm{C}_{\mathrm{s}}$ & 0.0 & 0.0 & 0.0 & 0.0 & 0.0 & 0.0 & 0.0 & 0.0 & 0.0 & 0.0 & 0.0 & 0.0 \\
\hline${ }^{2} \operatorname{Ir}\left(\mathrm{PH}_{3}\right)_{2} \mathrm{CO}$ & $\mathrm{C}_{1} a x, e q$ & 1.4 & 1.2 & 1.2 & 1.2 & 1.6 & 0.9 & 1.3 & 1.8 & 1.6 & 0.8 & 2.3 & 1.3 \\
\hline${ }^{4} \mathrm{Ir}\left(\mathrm{PH}_{3}\right)_{2} \mathrm{CO}$ & $\mathrm{C}_{\mathrm{s}} a x, e q$ & 45.4 & 44.5 & 44.7 & 45.5 & 43.6 & 48.8 & 46.2 & 48.3 & 48.2 & 54.2 & 51.2 & 42.4 \\
\hline${ }^{2} \operatorname{Ir}\left(\mathrm{PH}_{3}\right)_{2}(\mathrm{CO})_{2}$ & $\mathrm{C}_{2 \mathrm{v}} a x, a x$ & 0.0 & 0.0 & 0.0 & 0.0 & 0.0 & 0.0 & 0.0 & 0.0 & 0.0 & 0.0 & 0.0 & 0.0 \\
\hline${ }^{2} \mathrm{Ir}\left(\mathrm{PH}_{3}\right)_{2}(\mathrm{CO})_{2}$ & $\mathrm{C}_{2} a x, e q$ & 1.8 & 1.7 & 1.7 & 2.1 & 1.9 & 2.1 & 2.0 & 1.6 & 1.8 & 1.7 & 1.0 & 1.4 \\
\hline${ }^{4} \operatorname{Ir}\left(\mathrm{PH}_{3}\right)_{2}(\mathrm{CO})_{2}$ & $\mathrm{C}_{1} a x, e q$ & 63.4 & 63.0 & 63.1 & 63.3 & 64.0 & 64.2 & 64.9 & 68.1 & 68.4 & 69.3 & 82.8 & 66.3 \\
\hline${ }^{4} \operatorname{Ir}\left(\mathrm{PH}_{3}\right)_{2}(\mathrm{CO})_{2}$ & $\mathrm{C}_{2 \mathrm{v}} a x, a x$ & 62.0 & 61.5 & 61.5 & 62.9 & 66.3 & 62.8 & 65.0 & 72.5 & 70.6 & 67.9 & 83.1 & 69.3 \\
\hline${ }^{2} \operatorname{Ir}\left(\mathrm{PH}_{3}\right)_{3}$ & $\mathrm{C}_{\mathrm{s}}$ & 0.0 & 0.0 & 0.0 & 0.0 & 0.0 & 0.0 & 0.0 & 0.0 & 0.0 & 0.0 & 0.0 & 0.0 \\
\hline${ }^{4} \operatorname{Ir}\left(\mathrm{PH}_{3}\right)_{3}$ & $\mathrm{C}_{\mathrm{s}}$ & 53.4 & 53.2 & 53.4 & 50.4 & 47.7 & 58.8 & 51.6 & 51.6 & 52.8 & 58.8 & 77.0 & 64.7 \\
\hline${ }^{2} \mathrm{Ir}\left(\mathrm{PH}_{3}\right)_{3} \mathrm{CO}$ & $\mathrm{C}_{\mathrm{s}}$ & 0.0 & 0.0 & 0.0 & 0.0 & 0.0 & 0.0 & 0.0 & 0.0 & 0.0 & 0.0 & 0.0 & 0.0 \\
\hline${ }^{4} \mathrm{Ir}\left(\mathrm{PH}_{3}\right)_{3} \mathrm{CO}$ & $\mathrm{C}_{1}$ & 70.5 & 70.8 & 70.8 & 67.3 & 68.3 & 73.7 & 69.6 & 73.4 & 73.3 & 72.7 & 92.5 & 85.7 \\
\hline
\end{tabular}

${ }^{a}$ All energies are single-point energies at the SVWN5 optimized geometries. 
Table 2. LDEs of $\operatorname{Ir}\left(\mathrm{PH}_{3}\right)_{\mathrm{y}}(\mathrm{CO})_{\mathrm{z}}$ Isomers in $\mathrm{kcal} / \mathrm{mol}$, predicted by various electronic structure methods. ${ }^{\mathrm{a}}$

\begin{tabular}{|c|c|c|c|c|c|c|c|c|c|c|c|c|}
\hline Reaction & BP86 & PBE & PW91 & B3LYP & $\begin{array}{l}\text { CAM- } \\
\text { B3LYP }\end{array}$ & $\begin{array}{l}\text { B97 } \\
-\mathrm{D}\end{array}$ & B98 & $\omega B 97 X$ & $\begin{array}{c}\omega \mathrm{B} 97 \mathrm{X} \\
-\mathrm{D}\end{array}$ & M06 & MP2 & $\operatorname{CCSD}(\mathrm{T})$ \\
\hline $\begin{array}{c}{ }^{2} \operatorname{Ir}(\mathrm{CO})_{3} \rightarrow \\
{ }^{2} \operatorname{Ir}(\mathrm{CO})_{2}(\text { bent })+\mathrm{CO}\end{array}$ & 48.0 & 49.3 & 49.8 & 42.0 & 44.0 & 43.2 & 44.1 & 44.2 & 44.5 & 42.5 & 48.2 & 41.3 \\
\hline $\begin{array}{c}{ }^{2} \operatorname{Ir}(\mathrm{CO})_{3} \rightarrow \\
{ }^{2} \operatorname{Ir}(\mathrm{CO})_{2}(\text { linear })+\mathrm{CO}\end{array}$ & 46.5 & 49.2 & 49.2 & 33.4 & 33.3 & 40.8 & 35.9 & 33.0 & 35.6 & 36.2 & 64.0 & 51.2 \\
\hline $\begin{array}{c}{ }^{2} \mathrm{IrPH}_{3}(\mathrm{CO})_{2}(\mathrm{ax}) \rightarrow \\
\left.{ }^{2} \mathrm{Ir}(\mathrm{CO})_{2} \text { (bent }\right)+\mathrm{PH}_{3}\end{array}$ & 35.7 & 37.0 & 37.6 & 32.7 & 36.6 & 33.8 & 35.5 & 39.4 & 38.6 & 34.6 & 45.3 & 38.1 \\
\hline $\begin{array}{l}{ }^{2} \mathrm{IrPH}_{3}(\mathrm{CO})_{2}(\mathrm{ax}) \rightarrow \\
{ }^{2} \mathrm{IrPH}_{3} \mathrm{CO}(\text { bent })+\mathrm{CO}\end{array}$ & 53.8 & 55.3 & 55.7 & 47.1 & 49.0 & 48.0 & 49.4 & 49.6 & 50.1 & 47.5 & 55.6 & 46.5 \\
\hline $\begin{array}{c}{ }^{2} \mathrm{IrPH}_{3}(\mathrm{CO})_{2}(a x) \rightarrow \\
{ }^{2} \mathrm{IrPH}_{3} \mathrm{CO} \text { (linear) }+\mathrm{CO}\end{array}$ & 46.8 & 49.4 & 49.5 & 34.0 & 33.9 & 41.5 & 36.6 & 33.5 & 36.1 & 36.8 & 66.4 & 53.3 \\
\hline $\begin{array}{c}{ }^{2} \mathrm{IrPH}_{3}(\mathrm{CO})_{2}(e q) \rightarrow \\
{ }^{2} \mathrm{Ir}(\mathrm{CO})_{2} \text { (linear) }+\mathrm{PH}_{3}\end{array}$ & 33.1 & 35.8 & 36.0 & 22.5 & 23.8 & 30.9 & 25.7 & 25.5 & 27.4 & 26.5 & 58.6 & 45.8 \\
\hline $\begin{array}{c}{ }^{2} \mathrm{IrPH}_{3}(\mathrm{CO})_{2}(e q) \rightarrow \\
{ }_{2} \mathrm{IrPH}_{3} \mathrm{CO} \text { (bent) + CO }\end{array}$ & 52.5 & 54.1 & 54.6 & 45.5 & 46.8 & 47.5 & 48.0 & 46.9 & 47.9 & 45.7 & 53.0 & 44.3 \\
\hline $\begin{array}{l}{ }^{2} \mathrm{Ir}\left(\mathrm{PH}_{3}\right)_{2} \mathrm{CO}(a x, a x) \rightarrow \\
{ }^{2} \mathrm{IrPH} \\
3\end{array}$ & 36.2 & 37.6 & 38.1 & 32.3 & 36.3 & 33.6 & 35.3 & 39.2 & 38.6 & 34.0 & 47.2 & 38.4 \\
\hline $\begin{array}{l}{ }^{2} \mathrm{Ir}\left(\mathrm{PH}_{3}\right)_{2} \mathrm{CO}(a x, a x) \rightarrow \\
{ }^{2} \operatorname{Ir}\left(\mathrm{PH}_{3}\right)_{2}(\text { linear })+\mathrm{CO}\end{array}$ & 49.4 & 52.1 & 52.2 & 36.9 & 37.0 & 44.1 & 39.6 & 36.5 & 39.2 & 39.3 & 73.4 & 59.4 \\
\hline $\begin{array}{l}{ }^{2} \mathrm{Ir}\left(\mathrm{PH}_{3}\right)_{2} \mathrm{CO}(\text { ax }, e q) \rightarrow \\
{ }^{2} \mathrm{IrPH}_{3} \mathrm{CO} \text { (bent) }+\mathrm{PH}_{3}\end{array}$ & 34.8 & 36.4 & 36.9 & 31.1 & 34.6 & 32.6 & 34.1 & 37.4 & 37.0 & 33.2 & 44.9 & 37.1 \\
\hline $\begin{array}{l}{ }^{2} \mathrm{Ir}\left(\mathrm{PH}_{3}\right)_{2} \mathrm{CO}(a x, e q) \rightarrow \\
{ }^{2} \mathrm{IrPH}_{3} \mathrm{CO} \text { (linear) }+\mathrm{PH}_{3}\end{array}$ & 27.8 & 30.6 & 30.8 & 18.1 & 19.6 & 26.1 & 21.2 & 21.3 & 23.0 & 22.5 & 55.7 & 43.9 \\
\hline $\begin{array}{l}{ }^{2} \operatorname{Ir}\left(\mathrm{PH}_{3}\right)_{2} \mathrm{CO}(a x, e q) \rightarrow \\
{ }^{2} \operatorname{Ir}\left(\mathrm{PH}_{3}\right)_{2} \text { (bent) }+\mathrm{CO}\end{array}$ & 59.8 & 61.4 & 61.9 & 52.8 & 54.5 & 53.7 & 55.4 & 55.0 & 56.0 & 53.5 & 62.5 & 52.3 \\
\hline $\begin{array}{c}{ }^{2} \operatorname{Ir}\left(\mathrm{PH}_{3}\right)_{3} \rightarrow \\
\left.{ }^{2} \operatorname{Ir}\left(\mathrm{PH}_{3}\right)_{2} \text { (bent }\right)+\mathrm{PH}_{3}\end{array}$ & 37.9 & 39.7 & 40.2 & 33.4 & 36.9 & 35.0 & 36.6 & 40.0 & 39.8 & 35.7 & 49.5 & 39.8 \\
\hline $\begin{array}{c}{ }^{2} \operatorname{Ir}\left(\mathrm{PH}_{3}\right)_{3} \rightarrow \\
\left.{ }^{2} \operatorname{Ir}\left(\mathrm{PH}_{3}\right)_{2} \text { (linear) }\right)+\mathrm{PH}_{3}\end{array}$ & 26.2 & 29.1 & 29.3 & 16.3 & 17.8 & 24.5 & 19.6 & 19.6 & 21.4 & 20.7 & 58.0 & 45.6 \\
\hline
\end{tabular}


Table 2 (continued). LDEs of $\operatorname{Ir}\left(\mathrm{PH}_{3}\right)_{\mathrm{y}}(\mathrm{CO})_{\mathrm{z}}$ Isomers in $\mathrm{kcal} / \mathrm{mol}$, predicted by various electronic structure methods. ${ }^{\text {a }}$

\begin{tabular}{|c|c|c|c|c|c|c|c|c|c|c|c|c|}
\hline Reaction & BP86 & PBE & PW91 & B3LYP & $\begin{array}{l}\text { CAM- } \\
\text { B3LYP }\end{array}$ & $\begin{array}{l}\text { B97 } \\
-\mathrm{D}\end{array}$ & B98 & $\omega \mathrm{B} 97 \mathrm{X}$ & $\begin{array}{c}\omega \mathrm{B} 97 \mathrm{X} \\
-\mathrm{D}\end{array}$ & M06 & MP2 & $\operatorname{CCSD}(\mathrm{T})$ \\
\hline $\begin{array}{l}{ }^{2} \operatorname{Ir}(\mathrm{CO})_{4} \rightarrow \\
{ }^{2} \operatorname{Ir}(\mathrm{CO})_{3}+\mathrm{CO}\end{array}$ & 35.8 & 37.9 & 38.1 & 28.6 & 30.6 & 31.6 & 31.8 & 32.6 & 32.8 & 29.8 & 38.8 & 28.3 \\
\hline $\begin{array}{l}{ }^{2} \mathrm{IrPH}_{3}(\mathrm{CO})_{3} \rightarrow \\
{ }^{2} \operatorname{Ir}(\mathrm{CO})_{3}+\mathrm{PH}_{3}\end{array}$ & 24.4 & 26.5 & 26.8 & 19.7 & 23.4 & 23.1 & 23.7 & 27.7 & 27.2 & 22.2 & 36.5 & 25.3 \\
\hline $\begin{array}{c}{ }^{2} \mathrm{IrPH}_{3}(\mathrm{CO})_{3} \rightarrow \\
{ }^{2} \mathrm{IrPH}_{3}(\mathrm{CO})_{2}(\text { ax })+\mathrm{CO}\end{array}$ & 36.6 & 38.8 & 39.0 & 29.0 & 30.8 & 32.5 & 32.4 & 32.5 & 33.1 & 30 & 39.5 & 28.5 \\
\hline $\begin{array}{c}{ }^{2} \mathrm{IrPH}_{3}(\mathrm{CO})_{3} \rightarrow \\
{ }^{2} \mathrm{IrPH}_{3}(\mathrm{CO})_{2}(e q)+\mathrm{CO}\end{array}$ & 37.8 & 39.9 & 40.1 & 30.6 & 32.9 & 33.0 & 33.8 & 35.1 & 35.3 & 31.8 & 42.0 & 30.6 \\
\hline $\begin{array}{c}{ }^{2} \mathrm{Ir}(\mathrm{PH} 3)_{2}(\mathrm{CO})_{2}(a x, a x) \rightarrow \\
{ }^{2} \mathrm{IrPH}_{3}(\mathrm{CO})_{2}(e q)+\mathrm{PH}_{3}\end{array}$ & 22.4 & 24.7 & 25.0 & 17.8 & 21.3 & 21.6 & 21.9 & 25.4 & 25.2 & 20.3 & 34.4 & 23.7 \\
\hline $\begin{array}{l}{ }^{2} \mathrm{Ir}\left(\mathrm{PH}_{3}\right)_{2}(\mathrm{CO})_{2}(a x, a x) \rightarrow \\
{ }^{2} \operatorname{Ir}\left(\mathrm{PH}_{3}\right)_{2} \mathrm{CO}(a x, a x)+\mathrm{CO}\end{array}$ & 40.0 & 42.3 & 42.6 & 32.5 & 34.0 & 36.0 & 36.0 & 35.8 & 36.7 & 33.8 & 42.8 & 31.8 \\
\hline $\begin{array}{c}{ }^{2} \mathrm{Ir}(\mathrm{PH} 3)_{2}(\mathrm{CO})_{2}(a x, e q) \rightarrow \\
{ }^{2} \mathrm{IrPH}_{3}(\mathrm{CO})_{2}(a x)+\mathrm{PH}_{3}\end{array}$ & 20.6 & 23.0 & 23.3 & 15.7 & 19.4 & 19.5 & 19.9 & 23.8 & 23.4 & 18.6 & 33.4 & 22.2 \\
\hline $\begin{array}{c}{ }^{2} \mathrm{Ir}\left(\mathrm{PH}_{3}\right)_{2}(\mathrm{CO})_{2}(a x, e q) \rightarrow \\
{ }^{2} \operatorname{Ir}\left(\mathrm{PH}_{3}\right)_{2} \mathrm{CO}(a x, e q)+\mathrm{CO}\end{array}$ & 39.5 & 41.9 & 42.0 & 31.7 & 33.7 & 34.8 & 35.3 & 36.0 & 36.5 & 32.9 & 44.1 & 31.6 \\
\hline $\begin{array}{l}{ }^{2} \mathrm{Ir}\left(\mathrm{PH}_{3}\right)_{3} \mathrm{CO} \rightarrow \\
{ }^{2} \operatorname{Ir}\left(\mathrm{PH}_{3}\right)_{3}+\mathrm{CO} \\
\end{array}$ & 43.4 & 45.7 & 46.0 & 35.7 & 37.6 & 38.8 & 39.4 & 39.9 & 40.6 & 37.1 & 48.5 & 35.6 \\
\hline $\begin{array}{c}{ }^{2} \operatorname{Ir}\left(\mathrm{PH}_{3}\right)_{3} \mathrm{CO} \rightarrow \\
{ }^{2} \operatorname{Ir}\left(\mathrm{PH}_{3}\right)_{2} \mathrm{CO}(\text { ax }, e q)+\mathrm{PH}_{3}\end{array}$ & 21.5 & 24.0 & 24.3 & 16.3 & 20.0 & 20.1 & 20.7 & 24.8 & 24.4 & 19.3 & 35.5 & 23.1 \\
\hline $\begin{array}{c}{ }^{2} \operatorname{Ir}\left(\mathrm{PH}_{3}\right)_{3} \mathrm{CO} \rightarrow \\
{ }^{2} \operatorname{Ir}\left(\mathrm{PH}_{3}\right)_{2} \mathrm{CO}(a x, a x)+\mathrm{PH}_{3}\end{array}$ & 20.2 & 22.8 & 23.1 & 15.1 & 18.3 & 19.2 & 19.4 & 23 & 22.8 & 18.5 & 33.2 & 21.8 \\
\hline $\begin{array}{c}{ }^{2} \operatorname{Ir}\left(\mathrm{PH}_{3}\right)_{4} \rightarrow \\
{ }^{2} \operatorname{Ir}\left(\mathrm{PH}_{3}\right)_{3}+\mathrm{PH}_{3}\end{array}$ & 21.8 & 24.5 & 24.7 & 16.6 & 20.1 & 20.7 & 21.2 & 25.1 & 24.9 & 19.9 & 36.8 & 24.0 \\
\hline
\end{tabular}

${ }^{a}$ All energies are single-point energies at the SVWN5 optimized geometries. 
Table 3. Calculated NBO Charges on Ir, ${ }^{\mathrm{a}}$ Average C-O Stretching Frequencies $\left(v_{\mathrm{C}-\mathrm{O}}\right),{ }^{\mathrm{a}}$ Bond Distances $(r),{ }^{\mathrm{a}}$ and $\mathrm{LDEs}$ for $\operatorname{Ir}(\mathrm{PH})_{\mathrm{y}}(\mathrm{CO})_{\mathrm{z}}{ }^{\mathrm{b}}$

\begin{tabular}{|c|c|c|c|c|c|c|}
\hline \multirow[t]{2}{*}{ Property } & \multirow{2}{*}{$\frac{\operatorname{Ir}(\mathrm{CO})_{3}}{\mathbf{1 a}}$} & \multicolumn{2}{|c|}{$\mathrm{IrPH}_{3}(\mathrm{CO})_{2}$} & \multicolumn{2}{|c|}{$\mathrm{Ir}\left(\mathrm{PH}_{3}\right)_{2} \mathrm{CO}$} & \multirow{2}{*}{$\frac{\operatorname{Ir}\left(\mathrm{PH}_{3}\right)_{3}}{\mathbf{1 f}}$} \\
\hline & & $a x \mathbf{1 b}$ & $e q$ 1c & $a x, a x$ 1d & $a x, e q \mathbf{1 e}$ & \\
\hline Ir NBO charge & 0.02 & -0.13 & -0.13 & -0.31 & -0.38 & -0.59 \\
\hline Ir NBO 5d & 8.17 & 8.30 & 8.35 & 8.51 & 8.57 & 8.80 \\
\hline Average $v_{\mathrm{C}-\mathrm{O}}\left(\mathrm{cm}^{-1}\right)$ & 2054 & 2022 & 2011 & 1992 & 2002 & \\
\hline$r(\mathrm{C}-\mathrm{O})(a x, \AA)$ & 1.156 & 1.162 & 1.164 & & 1.167 & \\
\hline$r(\mathrm{C}-\mathrm{O})(\mathrm{eq}, \AA)$ & 1.159 & 1.165 & & 1.171 & & \\
\hline$r(\mathrm{Ir}-\mathrm{C})(\mathrm{ax}, \AA)$ & 1.901 & 1.865 & 1.877 & & 1.849 & \\
\hline$r(\mathrm{Ir}-\mathrm{C})(\mathrm{eq}, \AA)$ & 1.827 & 1.820 & & 1.813 & & \\
\hline $\mathrm{LDE}(\mathrm{CO})(a x, \mathrm{kcal} / \mathrm{mol})$ & 41.3 & 46.5 & 44.3 & & 52.3 & \\
\hline $\operatorname{LDE}(\mathrm{CO})(e q, \mathrm{kcal} / \mathrm{mol})$ & 51.2 & 53.3 & & 59.4 & & \\
\hline$r(\operatorname{Ir}-\mathrm{P})(a x, \AA)$ & & 2.315 & & 2.264 & 2.294 & 2.243 \\
\hline$r(\mathrm{Ir}-\mathrm{P})(e q, \AA)$ & & & 2.230 & & 2.211 & 2.198 \\
\hline $\operatorname{LDE}\left(\mathrm{PH}_{3}\right)(a x, \mathrm{kcal} / \mathrm{mol})$ & & 38.1 & & 38.4 & 37.1 & 39.8 \\
\hline $\operatorname{LDE}\left(\mathrm{PH}_{3}\right)(e q, \mathrm{kcal} / \mathrm{mol})$ & & & 45.8 & & 43.9 & 45.6 \\
\hline
\end{tabular}


Table 3 (continued). Calculated NBO Charges on Ir, ${ }^{a}$ Average C-O Stretching Frequencies $\left(v_{\mathrm{C}-\mathrm{O}}\right),{ }^{\mathrm{a}}$ Bond Distances $(r),{ }^{\mathrm{a}}$ and $\mathrm{LDEs}$ for $\operatorname{Ir}\left(\mathrm{PH}_{3}\right)_{\mathrm{y}}(\mathrm{CO})_{\mathrm{z}}{ }^{\mathrm{b}}$

\begin{tabular}{|c|c|c|c|c|c|c|}
\hline \multirow[t]{2}{*}{ Property } & $\operatorname{Ir}(\mathrm{CO})_{4}$ & $\mathrm{IrPH}_{3}(\mathrm{CO})_{3}$ & $\operatorname{Ir}\left(\mathrm{PH}_{3}\right)_{2}(\mathrm{CO})_{2}$ & $\operatorname{Ir}\left(\mathrm{PH}_{3}\right)_{2}(\mathrm{CO})_{2}$ & $\mathrm{Ir}\left(\mathrm{PH}_{3}\right)_{3} \mathrm{CO}$ & $\operatorname{Ir}\left(\mathrm{PH}_{3}\right)_{4}$ \\
\hline & 19 & 1h & $a x, a x \mathbf{1 i}$ & $a x, e q \mathbf{1 j}$ & $\mathbf{1 k}$ & 11 \\
\hline Ir NBO charge & 0.16 & 0.07 & -0.15 & -0.09 & -0.29 & -0.51 \\
\hline Ir NBO 5d & 8.21 & 8.27 & 8.44 & 8.43 & 8.63 & 8.87 \\
\hline Average $v_{\mathrm{C}-\mathrm{O}}\left(\mathrm{cm}^{-1}\right)$ & 2054 & 2023 & 1988 & 2000 & 1975 & \\
\hline$r(\mathrm{C}-\mathrm{O})(a x, \AA)$ & 1.156 & 1.160 & & 1.166 & & \\
\hline$r(\mathrm{C}-\mathrm{O})(e q, \AA)$ & & 1.162 & 1.168 & & 1.170 & \\
\hline$r(\operatorname{Ir}-\mathrm{C})(a x, \AA)$ & 1.901 & 1.874 & & 1.867 & & \\
\hline$r(\mathrm{Ir}-\mathrm{C})(e q, \AA)$ & & 1.892 & 1.879 & & 1.858 & \\
\hline $\operatorname{LDE}(\mathrm{CO})(a x, \mathrm{kcal} / \mathrm{mol})$ & 28.3 & 30.6 & & 31.6 & & \\
\hline $\operatorname{LDE}(\mathrm{CO})(e q, \mathrm{kcal} / \mathrm{mol})$ & & 28.5 & 31.8 & & 35.6 & \\
\hline$r(\operatorname{Ir}-\mathrm{P})(a x, \AA)$ & & 2.316 & 2.285 & 2.300 & 2.270 & 2.254 \\
\hline$r(\mathrm{Ir}-\mathrm{P})(e q, \AA)$ & & & & & 2.281 & \\
\hline $\operatorname{LDE}\left(\mathrm{PH}_{3}\right)(a x, \mathrm{kcal} / \mathrm{mol})$ & & 25.3 & 23.7 & 22.2 & 23.1 & 24.0 \\
\hline $\operatorname{LDE}\left(\mathrm{PH}_{3}\right)(e q, \mathrm{kcal} / \mathrm{mol})$ & & & & & 21.8 & \\
\hline
\end{tabular}

${ }^{a}$ All bond distances, frequencies, and NBO charges are calculated at the SVWN5 level.

${ }^{\mathrm{b}}$ All LDEs are calculated by $\operatorname{CCSD}(\mathrm{T})$ at the SVWN5 optimized geometries 
Table 4. Relative Energies of $\operatorname{Ir}_{2}\left(\mathrm{PH}_{3}\right)_{\mathrm{y}}(\mathrm{CO})_{\mathrm{z}}$ Isomers in $\mathrm{kcal} / \mathrm{mol}$, predicted by various electronic structure methods. ${ }^{\mathrm{a}}$

\begin{tabular}{|c|c|c|c|c|c|c|c|c|c|c|c|c|}
\hline Molecule & BP86 & PBE & PW91 & B3LYP & \begin{tabular}{|l|} 
CAM- \\
B3LYP
\end{tabular} & B97-D & B98 & $\omega \mathrm{B} 97 \mathrm{X}$ & $\begin{array}{c}\omega \mathrm{B} 97 \mathrm{X}- \\
\mathrm{D} \\
\end{array}$ & M06 & MP2 & $\operatorname{CCSD}(\mathrm{T})$ \\
\hline $\mathrm{Ir}_{2}(\mathrm{CO})_{7} \mathbf{2 b}$ & 0.0 & 0.0 & 0.0 & 0.0 & 0.0 & 0.0 & 0.0 & 0.0 & 0.0 & 0.0 & 0.0 & 0.0 \\
\hline $\mathrm{Ir}_{2}(\mathrm{CO})_{7} \mathbf{2 a}$ & 7.2 & 6.6 & 6.7 & 10.7 & 11.5 & 12.1 & 10.0 & 12.2 & 11.7 & 8.3 & 5.7 & 11.2 \\
\hline $\mathrm{Ir}_{2}(\mathrm{CO})_{7} \mathbf{2 c}$ & 29.9 & 29.8 & 29.7 & 29.5 & 29.7 & 24.0 & 29.5 & 29.7 & 29.5 & 30.4 & 29.0 & 28.0 \\
\hline $\mathrm{Ir}_{2} \mathrm{PH}_{3}(\mathrm{CO})_{7} \mathbf{2 f}$ & 0.0 & 0.0 & 0.0 & 0.0 & 0.0 & 0.0 & 0.0 & 0.0 & 0.0 & 0.0 & 0.0 & 0.0 \\
\hline $\mathrm{Ir}_{2} \mathrm{PH}_{3}(\mathrm{CO})_{7} \mathbf{2 d}$ & -2.2 & -2.7 & -2.5 & 1.3 & 1.8 & 5.7 & 0.6 & 2.5 & 1.6 & 0.0 & 1.9 & 2.7 \\
\hline $\mathrm{Ir}_{2} \mathrm{PH}_{3}(\mathrm{CO})_{7} \mathbf{2 e}$ & -0.9 & -1.1 & -1.1 & 1.3 & 2.2 & 4.2 & 1.3 & 2.6 & 1.9 & 0.0 & 3.9 & 3.0 \\
\hline $\mathrm{Ir}_{2} \mathrm{PH}_{3}(\mathrm{CO})_{6} \mathbf{2 i}$ & 0.0 & 0.0 & 0.0 & 0.0 & 0.0 & 0.0 & 0.0 & 0.0 & 0.0 & 0.0 & 0.0 & 0.0 \\
\hline $\mathrm{Ir}_{2} \mathrm{PH}_{3}(\mathrm{CO})_{6} \mathbf{2 j}$ & 1.7 & 0.9 & 1.2 & 7.2 & 8.5 & 6.7 & 6.3 & 9.2 & 8.2 & 7.5 & 3.0 & 8.3 \\
\hline $\mathrm{Ir}_{2} \mathrm{PH}_{3}(\mathrm{CO})_{6} \mathbf{2 h}$ & 4.7 & 3.9 & 4.1 & 8.9 & 9.8 & 9.6 & 8.2 & 10.7 & 9.9 & 6.6 & 4.3 & 10.0 \\
\hline $\mathrm{Ir}_{2} \mathrm{PH}_{3}(\mathrm{CO})_{6} \mathbf{2 g}$ & 9.4 & 8.9 & 9.2 & 15.1 & 16.3 & 13.4 & 14.4 & 16.8 & 16.1 & 19.4 & 1.6 & 14.9 \\
\hline $\mathrm{Ir}_{2} \mathrm{PH}_{3}(\mathrm{CO})_{6} \mathbf{2 k}$ & 29.6 & 29.7 & 29.6 & 30.1 & 31.0 & 23.6 & 30.4 & 31.9 & 31.2 & 32.7 & 29.8 & 30.0 \\
\hline $\mathrm{Ir}_{2}\left(\mathrm{PH}_{3}\right)_{2}(\mathrm{CO})_{6} 2 \mathrm{l}$ & 0.0 & 0.0 & 0.0 & 0.0 & 0.0 & 0.0 & 0.0 & 0.0 & 0.0 & 0.0 & 0.0 & 0.0 \\
\hline $\mathrm{Ir}_{2}\left(\mathrm{PH}_{3}\right)_{2}(\mathrm{CO})_{6} \mathbf{2 0}$ & 3.1 & 4.0 & 3.8 & 0.1 & 0.6 & -4.5 & 0.9 & 0.9 & 1.4 & 3.6 & -0.6 & 0.0 \\
\hline $\mathrm{Ir}_{2}\left(\mathrm{PH}_{3}\right)_{2}(\mathrm{CO})_{6} \mathbf{2 m}$ & 2.2 & 2.7 & 2.6 & 0.8 & 1.7 & -1.2 & 1.6 & 1.5 & 1.7 & 1.6 & 2.4 & 1.2 \\
\hline $\mathrm{Ir}_{2}\left(\mathrm{PH}_{3}\right)_{2}(\mathrm{CO})_{6} \mathbf{2 n}$ & 12.6 & 12.3 & 12.3 & 15.5 & 17.1 & 13.0 & 15.1 & 17.6 & 16.5 & 13.5 & 9.1 & 14.9 \\
\hline $\mathrm{Ir}_{2}\left(\mathrm{PH}_{3}\right)_{2}(\mathrm{CO})_{5} \mathbf{2 r}$ & 0.0 & 0.0 & 0.0 & 0.0 & 0.0 & 0.0 & 0.0 & 0.0 & 0.0 & 0.0 & 0.0 & 0.0 \\
\hline $\mathrm{Ir}_{2}\left(\mathrm{PH}_{3}\right)_{2}(\mathrm{CO})_{5} \mathbf{2 t}$ & 12.0 & 13.4 & 12.9 & 6.6 & 10.6 & 4.1 & 9.5 & 12.9 & 12.5 & 5.3 & 14.4 & 12.8 \\
\hline $\mathrm{Ir}_{2}\left(\mathrm{PH}_{3}\right)_{2}(\mathrm{CO})_{5} \mathbf{2 s}$ & 11.5 & 11.8 & 11.6 & 12.0 & 17.0 & 10.8 & 13.8 & 19.3 & 17.7 & 6.9 & 15.4 & 18.7 \\
\hline $\mathrm{Ir}_{2}\left(\mathrm{PH}_{3}\right)_{2}(\mathrm{CO})_{5} \mathbf{2 q}$ & 12.4 & 12.7 & 12.5 & 12.2 & 16.7 & 11.2 & 14.1 & 18.9 & 17.6 & 7.4 & 17.2 & 18.8 \\
\hline $\mathrm{Ir}_{2}\left(\mathrm{PH}_{3}\right)_{2}(\mathrm{CO})_{5} \mathbf{2 p}$ & 18.4 & 19.0 & 18.8 & 18.7 & 23.1 & 15.7 & 20.6 & 25.2 & 24.1 & 18.7 & 13.8 & 23.7 \\
\hline
\end{tabular}

${ }^{a}$ All energies are single-point energies from SVWN5 optimized geometries. 
Table 5. $\operatorname{Ir}_{2}\left(\mathrm{PH}_{3}\right)_{\mathrm{y}}(\mathrm{CO})_{\mathrm{z}}$ LDEs in $\mathrm{kcal} / \mathrm{mol}$, predicted by various electronic structure methods. ${ }^{\mathrm{a}}$

\begin{tabular}{|c|c|c|c|c|c|c|c|c|c|c|c|c|c|}
\hline Reaction & $\begin{array}{l}\text { Leaving } \\
\text { Ligand }\end{array}$ & BP86 & PBE & PW91 & B3LYP & $\begin{array}{l}\text { CAM- } \\
\text { B3LYP }\end{array}$ & B97-D & B98 & $\omega B 97 X$ & $\begin{array}{c}\omega \mathrm{B} 97 \mathrm{X} \\
-\mathrm{D}\end{array}$ & M06 & MP2 & $\operatorname{CCSD}(\mathrm{T})$ \\
\hline 1 & $\mathrm{PH}_{3}$ & 21.2 & 23.8 & 24.1 & 18.2 & 23.8 & 23.5 & 22.5 & 27.7 & 28.4 & 18.7 & 36.3 & 28.6 \\
\hline 2 & $\mathrm{PH}_{3}$ & 12.7 & 15.6 & 16.0 & 7.5 & 11.9 & 12.9 & 11.7 & 15.4 & 16.3 & 10.4 & 28.6 & 17.1 \\
\hline 3 & $\mathrm{PH}_{3}$ & 41.7 & 44.2 & 44.6 & 38.3 & 43.8 & 41.1 & 42.6 & 47.7 & 47.7 & 40.9 & 61.5 & 48.1 \\
\hline 4 & $\mathrm{CO} b r i$ & 32.4 & 34.9 & 35.3 & 27.8 & 31.6 & 30.2 & 31.2 & 32.7 & 34.0 & 32.5 & 31.3 & 30.8 \\
\hline 5 & $\mathrm{CO}$ ter & 27.7 & 29.9 & 30.1 & 21.6 & 25.1 & 26.4 & 25.1 & 26.6 & 27.7 & 19.8 & 34.0 & 25.8 \\
\hline 6 & $\mathrm{CO}$ & 21.7 & 24.5 & 24.6 & 12.7 & 15.0 & 18.3 & 16.1 & 15.8 & 17.5 & 13.1 & 27.8 & 15.6 \\
\hline 7 & $\mathrm{CO}$ & 50.4 & 53.0 & 53.1 & 44.1 & 48.2 & 46.1 & 47.8 & 50.3 & 50.6 & 45.8 & 61.5 & 48.6 \\
\hline 8 & $\mathrm{PH}_{3}$ & 18.9 & 21.5 & 21.9 & 16.2 & 22.2 & 21.3 & 20.4 & 26.4 & 26.8 & 17.3 & 34.5 & 27.4 \\
\hline 9 & $\mathrm{PH}_{3}$ & 12.0 & 14.9 & 15.3 & 6.5 & 10.7 & 13.0 & 10.6 & 14.3 & 15.2 & 9.1 & 27.9 & 16.2 \\
\hline 10 & $\mathrm{PH}_{3}$ & 3.3 & 6.3 & 6.8 & -1.0 & 3.8 & 5.4 & 3.4 & 7.5 & 8.6 & 4.7 & 24.2 & 10.9 \\
\hline 11 & $\mathrm{PH}_{3}$ & 40.7 & 43.3 & 43.6 & 37.3 & 42.8 & 39.8 & 41.7 & 46.7 & 46.7 & 39.8 & 60.6 & 47.5 \\
\hline 12 & $\mathrm{CO} b r i$ & 34.2 & 36.9 & 37.3 & 30.0 & 34.0 & 31.9 & 33.4 & 35.5 & 36.8 & 35.3 & 32.4 & 33.2 \\
\hline 13 & $\mathrm{CO}$ ter & 28.2 & 30.7 & 31.0 & 23.5 & 27.6 & 27.5 & 26.9 & 29.3 & 30.3 & 24.0 & 35.9 & 28.3 \\
\hline 14 & $\mathrm{CO}$ & 26.0 & 28.0 & 28.4 & 22.7 & 25.9 & 28.7 & 25.3 & 27.8 & 28.6 & 22.4 & 33.5 & 27.1 \\
\hline 15 & $\mathrm{CO} b r i$ & 3.2 & 5.6 & 6.1 & -4.2 & -6.2 & 3.3 & -2.3 & -7.2 & -3.8 & 3.1 & 9.0 & -5.3 \\
\hline 16 & $\mathrm{CO}$ ter & 14.7 & 17.4 & 17.8 & 7.8 & 10.8 & 14.1 & 11.5 & 12.0 & 13.9 & 10.0 & 25.0 & 13.4 \\
\hline 17 & $\mathrm{CO}$ & 24.7 & 27.3 & 27.6 & 17.8 & 20.9 & 24.9 & 21.3 & 22.3 & 23.7 & 18.4 & 33.7 & 22.4 \\
\hline
\end{tabular}

${ }^{a}$ All energies are single point energies from SVWN5 optimized geometries. Reaction numbering from Figure 3. 
Table 6. Calculated NBO Charges on Ir, ${ }^{a}$ Average C-O Stretching Frequencies ( $\left.v_{\mathrm{C}-\mathrm{O}}\right),{ }^{\mathrm{a}} \mathrm{Ir}-\mathrm{Ir}$ Bond Energies (BDE), ${ }^{\mathrm{b}}$ Bond Distances $(r)^{\mathrm{a}}$, and LDEs for $\operatorname{Ir}_{2}\left(\mathrm{PH}_{3}\right)_{\mathrm{y}}(\mathrm{CO})_{\mathrm{z}}{ }^{\mathrm{b}}$

\begin{tabular}{|l|c|c|c|}
\hline Property & $\mathrm{Ir}_{2}(\mathrm{CO})_{8} \mathrm{C}_{2 \mathrm{v}}$ & $\mathrm{Ir}_{2} \mathrm{PH}_{3}(\mathrm{CO})_{7}(\mathbf{2 d})$ & $\mathrm{Ir}_{2}\left(\mathrm{PH}_{3}\right)_{2}(\mathrm{CO})_{6}(\mathbf{2 l})$ \\
\hline Ir NBO charge & 0.22 & $0.23^{\mathrm{c}}, 0.06^{\mathrm{d}}$ & 0.07 \\
\hline $\mathrm{Ir}$ NBO 5d & 8.21 & $8.20^{\mathrm{c}}, 8.33^{\mathrm{d}}$ & 8.32 \\
\hline$r(\mathrm{Ir}-\mathrm{Ir})(\AA)$ & 2.774 & 2.760 & 2.744 \\
\hline BDE $(\mathrm{Ir}-\mathrm{Ir})(\mathrm{kcal} / \mathrm{mol})$ & 45.7 & 52.7 & 57.2 \\
\hline Average $v_{\mathrm{C}-\mathrm{O}}\left(\mathrm{cm}^{-1}\right)$ & 2026 & 2002 & 1972 \\
\hline$r(\mathrm{C}-\mathrm{O})($ ter,$\AA)$ & 1.152 & 1.154 & 1.156 \\
\hline$r(\mathrm{C}-\mathrm{O})($ bri,$\AA)$ & 1.180 & 1.186 & 1.193 \\
\hline$r(\mathrm{Ir}-\mathrm{C})^{\mathrm{c}}($ ter,$\AA)$ & 1.927 & $1.925^{\mathrm{c}}, 1.916^{\mathrm{d}}$ & 1.914 \\
\hline$r(\mathrm{Ir}-\mathrm{C})^{\mathrm{c}}($ bri,$\AA)$ & 2.090 & $2.106^{\mathrm{c}}, 2.061^{\mathrm{d}}$ & 2.075 \\
\hline $\mathrm{LDE}($ ter $\mathrm{CO})(\mathrm{kcal} / \mathrm{mol})$ & & 25.8 & 28.3 \\
\hline $\mathrm{LDE}($ bri CO$)(\mathrm{kcal} / \mathrm{mol})$ & & 30.8 & 33.2 \\
\hline$r(\mathrm{Ir}-\mathrm{P})(\AA)$ & & 2.309 & 2.300 \\
\hline $\mathrm{LDE}\left(\mathrm{PH}{ }_{3}\right)(\mathrm{kcal} / \mathrm{mol})$ & & 28.6 & 27.4 \\
\hline
\end{tabular}

${ }^{a}$ All bond distances, frequencies, and NBO charges are calculated at the SVWN5 level.

${ }^{\mathrm{b}}$ All BDEs and LDEs are calculated by $\mathrm{CCSD}(\mathrm{T})$ at the SVWN5 optimized geometries.

${ }^{\mathrm{c}}$ Ir not bonded to $\mathrm{PH}_{3}$.

${ }^{\mathrm{d}}$ Ir bonded to $\mathrm{PH}_{3}$. 
Table 7. Relative Energies of unsaturated Ir4(PH3)y(CO)z Isomers in kcal/mol at the $\omega$ B97x-D level. ${ }^{a}$

\begin{tabular}{|c|c|c|c|c|c|c|c|c|c|c|c|c|c|c|c|c|c|c|c|}
\hline $\mathrm{Ir}_{4}(\mathrm{CO})_{11}$ & $\mathbf{3 a}$ & $\mathbf{3 b}$ & $3 c$ & 3d & $3 e$ & & & & & & & & & & & & & & \\
\hline $\begin{array}{c}\text { Rel. } \\
\text { Energies }\end{array}$ & 13.2 & 3.3 & 1.1 & 0.7 & 0.0 & & & & & & & & & & & & & & \\
\hline $\mathrm{Ir}_{4} \mathrm{PH}_{3}(\mathrm{CO})_{10}$ & $5 \mathbf{5 a}$ & $\mathbf{5 b}$ & $5 c$ & $5 d$ & $5 e$ & $5 f$ & $5 g$ & $5 \mathrm{hh}$ & $5 \mathbf{i}$ & $5 \mathbf{j}$ & $5 \mathbf{k}$ & 51 & $5 \mathrm{~m}$ & $5 n$ & 50 & $5 p$ & $5 q$ & $5 r$ & $5 s$ \\
\hline $\begin{array}{c}\text { Rel. } \\
\text { Energies }\end{array}$ & 24.9 & 25.9 & 35.9 & 29.5 & 26.3 & 21.4 & 22.2 & 35.0 & 33.1 & 27.6 & 0.0 & 42.9 & 29.5 & 26.0 & 23.8 & 36.9 & 26.5 & 24.1 & 20.3 \\
\hline $\mathrm{Ir}_{4}\left(\mathrm{PH}_{3}\right)_{2}(\mathrm{CO})_{9}$ & $7 a$ & $7 \mathrm{~b}$ & $7 c$ & $7 d$ & $7 e$ & $7 f$ & $7 g$ & $7 \mathrm{~h}$ & $7 \mathbf{i}$ & $7 \mathbf{j}$ & $7 \mathrm{k}$ & 71 & $7 \mathrm{~m}$ & $7 n$ & 70 & $7 p$ & & & \\
\hline $\begin{array}{c}\text { Rel. } \\
\text { Energies }\end{array}$ & 19.9 & 26.5 & 34.2 & 25.9 & 29.5 & 35.5 & 33.4 & 0.0 & 25.6 & 24.6 & 36.6 & 23.9 & 22.7 & 20.3 & 20.4 & 23.6 & & & \\
\hline $\mathrm{Ir}_{4}\left(\mathrm{PH}_{3}\right)_{3}(\mathrm{CO})_{8}$ & $9 a$ & $9 \mathrm{~b}$ & $9 \mathrm{c}$ & 9d & $9 e$ & 9f & $9 g$ & $9 \mathrm{~h}$ & $9 \mathbf{i}$ & $9 \mathbf{j}$ & $9 \mathbf{k}$ & 91 & $9 \mathrm{~m}$ & 9n & 9o & $9 p$ & $9 q$ & $9 r$ & \\
\hline $\begin{array}{c}\text { Rel. } \\
\text { Energies }\end{array}$ & 18.0 & 18.0 & 25.5 & 39.7 & 30.8 & 34.5 & 20.9 & 23.9 & 31.2 & 21.4 & 21.8 & 38.6 & 32.9 & 2.3 & 23.5 & 27.3 & 0.0 & 21.3 & \\
\hline $\mathrm{Ir}_{4}\left(\mathrm{PH}_{3}\right)_{4}(\mathrm{CO})_{7}$ & $11 a$ & $11 b$ & 11c & 11d & $11 \mathrm{e}$ & 11f & $11 \mathrm{~g}$ & $11 \mathrm{~h}$ & $11 \mathrm{i}$ & 11j & & & & & & & & & \\
\hline $\begin{array}{c}\text { Rel. } \\
\text { Energies }\end{array}$ & 23.1 & 9.8 & 36.5 & 22.6 & 19.5 & 34.8 & 24.6 & 0.0 & 20.5 & 6.1 & & & & & & & & & \\
\hline
\end{tabular}

a $\mathbf{3 a}-\mathbf{1 1} \mathbf{j}$ are the isomers shown in Figure 4. 
Table 8. Average Ir NBO Charges, ${ }^{\mathrm{a}} \Delta \mathrm{NBO}$, and Relative Energies $(\Delta \mathrm{E}, \mathrm{kcal} / \mathrm{mol}) \mathrm{b}$ for $\operatorname{Ir}_{4}\left(\mathrm{PH}_{3}\right)_{\mathrm{y}}(\mathrm{CO})_{\mathrm{z}}$ Isomers, $\mathrm{y}+\mathrm{z}=12$.

\begin{tabular}{|c|c|c|c|c|c|c|}
\hline Property/Molecule & \multicolumn{3}{|c|}{$\mathrm{C}_{3 \mathrm{v}}$} & \multicolumn{2}{c|}{$\mathrm{T}_{\mathrm{d}}$} \\
\hline $\mathrm{Ir}_{4}(\mathrm{CO})_{12}$ & & & & & 0.08 & \\
\hline Ave NBO charge & 0.10 & & & & 0.00 & \\
\hline$\Delta \mathrm{NBO}$ & -0.02 & & & & 0.0 & \\
\hline$\Delta \mathrm{E}$ & 2.8 & & & & $\mathbf{4 e}$ & \\
\hline $\mathrm{Ir}_{4} \mathrm{PH}_{3}(\mathrm{CO})_{11}$ & $\mathbf{4 a}$ & $\mathbf{4 b}$ & $\mathbf{4 c}$ & $\mathbf{4 d}$ & 0.06 & \\
\hline Ave NBO charge & 0.07 & 0.07 & 0.06 & 0.09 & 0.02 & \\
\hline$\Delta \mathrm{NBO}$ & 0.01 & 0.01 & 0.02 & -0.01 & 0.0 & \\
\hline$\Delta \mathrm{E}$ & 0.3 & 1.1 & 10.0 & 1.5 & $\mathbf{6 d}$ & $\mathbf{6 e}$ \\
\hline $\mathrm{Ir}_{4}\left(\mathrm{PH}_{3}\right)_{2}(\mathrm{CO})_{10}$ & $\mathbf{6 a}$ & $\mathbf{6 b}$ & $\mathbf{6 c}$ & & 0.03 & 0.03 \\
\hline Ave $\mathrm{NBO}$ charge & 0.05 & 0.04 & 0.05 & & 0.05 & 0.05 \\
\hline$\Delta \mathrm{NBO}$ & 0.03 & 0.04 & 0.03 & & 0.8 & 0.6 \\
\hline$\Delta \mathrm{E}$ & 0.0 & 8.2 & 1.1 & & $\mathbf{8 d}$ & $\mathbf{8 e}$ \\
\hline $\mathrm{Ir}_{4}(\mathrm{PH})_{3}(\mathrm{CO})_{9}$ & $\mathbf{8 a}$ & $\mathbf{8 b}$ & $\mathbf{8 c}$ & & -0.01 & -0.04 \\
\hline $\mathrm{Ave} \mathrm{NBO}$ charge $_{\mathrm{N}}$ & 0.02 & 0.00 & 0.00 & & 0.09 & 0.12 \\
\hline$\Delta \mathrm{NBO}$ & 0.06 & 0.08 & 0.08 & & 3.4 & 2.4 \\
\hline$\Delta \mathrm{E}$ & 0.1 & 0.0 & 6.8 & & $\mathbf{1 0 d}$ & \\
\hline $\mathrm{Ir}_{4}(\mathrm{PH})_{4}(\mathrm{CO})_{8}$ & $\mathbf{1 0 a}$ & $\mathbf{1 0 b}$ & $\mathbf{1 0 c}$ & & -0.04 & \\
\hline $\mathrm{Ave} \mathrm{NBO}$ charge $_{\Delta \mathrm{NBO}}^{-0.02}$ & 0.10 & -0.02 & -0.03 & & 0.12 & \\
\hline$\Delta \mathrm{E}$ & 0.0 & 0.10 & 0.11 & & 2.6 & \\
\hline
\end{tabular}

${ }^{\text {a }}$ All NBO charges are calculated at the SVWN5 level.

${ }^{\mathrm{b}}$ All relative energies are calculated at the $\omega \mathrm{B} 97 \mathrm{x}-\mathrm{D}$ level at the SVWN5 optimized geometries. 
Table 9. $\mathrm{PH}_{3}$ and $\mathrm{CO}$ LDEs for $\mathrm{Ir}_{\mathrm{x}}\left(\mathrm{PH}_{3}\right)_{\mathrm{y}}(\mathrm{CO})_{\mathrm{z}}$ clusters in $\mathrm{kcal} / \mathrm{mol}$ at the $\omega \mathrm{B} 97 \mathrm{x}-\mathrm{D}$ level. ${ }^{\mathrm{a}}$

\begin{tabular}{|c|c|c|c|c|c|c|c|}
\hline Molecule & $\mathrm{C}_{3 \mathrm{v}} e q$ & $\mathrm{C}_{3 \mathrm{v}} a x$ & $\mathrm{C}_{3 \mathrm{v}}$ api & $\mathrm{C}_{3 \mathrm{v}} b r i$ & $\begin{array}{c}\mathrm{C}_{3 \mathrm{v}} \\
\text { H-trans }\end{array}$ & $\mathrm{T}_{\mathrm{d}}$ & $\begin{array}{c}T_{d} \\
\text { H-trans }\end{array}$ \\
\hline \multicolumn{8}{|c|}{$\mathrm{PH}_{3}$} \\
\hline $\mathrm{Ir}_{4} \mathrm{PH}_{3}(\mathrm{CO})_{11}$ & $50.8(\mathbf{4 a})$ & $40.2(\mathbf{4 b})$ & $37.1(4 d)$ & $29.1(4 c)$ & & $38.0(\mathbf{4 e})$ & \\
\hline \multirow[t]{2}{*}{$\mathrm{Ir}_{4}\left(\mathrm{PH}_{3}\right)_{2}(\mathrm{CO})_{10}$} & $49.4(\mathbf{6 a})$ & $40.7(\mathbf{6 a})$ & $33.8(\mathbf{6 b})$ & $30.0(\mathbf{6 b})$ & & $37.8(\mathbf{6 d})$ & \\
\hline & & $39.8(\mathbf{6 c})$ & & & & $34.1(\mathbf{6 e})$ & \\
\hline \multirow[t]{2}{*}{$\mathrm{Ir}_{4}\left(\mathrm{PH}_{3}\right)_{3}(\mathrm{CO})_{9}$} & $49.7(\mathbf{8 a})$ & $41.5(\mathbf{8 a})$ & $32.7(\mathbf{8 c})$ & $29.2(8 c)$ & & $34.9^{b}(\mathbf{8 d})$ & \\
\hline & $51.1(8 b)$ & & & & & $33.6^{\mathrm{b}}(\mathbf{8 e})$ & \\
\hline \multirow[t]{2}{*}{$\mathrm{Ir}_{4}\left(\mathrm{PH}_{3}\right)_{4}(\mathrm{CO})_{8}$} & $48.5(\mathbf{1 0 a})$ & $35.4(\mathbf{1 0 b})$ & $32.0(\mathbf{1 0 a})$ & $28.3(\mathbf{1 0 c})$ & & $32.7(\mathbf{1 0 d})$ & \\
\hline & $45.2(\mathbf{1 0 c})$ & & & & & & \\
\hline \multicolumn{8}{|c|}{$\mathrm{CO}$} \\
\hline $\mathrm{Ir}_{4}(\mathrm{CO})_{12}$ & 52.6 & 42.7 & 40.1 & 40.5 & & 39.4 & \\
\hline \multirow[t]{2}{*}{$\mathrm{Ir}_{4} \mathrm{PH}_{3}(\mathrm{CO})_{11}$} & $53.5(4 a)$ & $\begin{array}{l}47.1(\mathbf{4 a}) \\
43.9(\mathbf{4 a})\end{array}$ & $42.5(4 \mathbf{a})$ & $43.5(\mathbf{4 a})$ & & $42.1(4 \mathbf{e})$ & \\
\hline & $51.9(4 b)$ & & $38.3(4 \mathbf{b})$ & $39.1(4 b)$ & & $38.2(\mathbf{4 e})$ & \\
\hline \multirow[t]{2}{*}{$\mathrm{Ir}_{4} \mathrm{PH}_{3}(\mathrm{CO})_{11}$} & $50.9(4 c)$ & & $\begin{array}{l}41.1(4 c) \\
35.6(4 c)\end{array}$ & & $8.0(4 c)$ & & \\
\hline & $53.3(4 d)$ & $43.0(\mathbf{4 d})$ & $46.0(4 d)$ & $\begin{array}{l}42.5(\mathbf{4 d}) \\
40.3(\mathbf{4 d})\end{array}$ & & & \\
\hline \multirow[t]{3}{*}{$\mathrm{Ir}_{4}\left(\mathrm{PH}_{3}\right)_{2}(\mathrm{CO})_{10}$} & $53.5(\mathbf{6 a})$ & $48.9(\mathbf{6 a})$ & $39.2(\mathbf{6 a})$ & $45.9(\mathbf{6 a})$ & $11.2(\mathbf{6 b})$ & $41.2^{\mathrm{c}}(\mathbf{6 d})$ & \\
\hline & $36.8^{\mathrm{b}}(\mathbf{6 b})$ & & $44.6(\mathbf{6 b})$ & & & $38.9(\mathbf{6 d})$ & \\
\hline & $54.9(\mathbf{6 c})$ & & $42.9(\mathbf{6 c})$ & $41.0(\mathbf{6 c})$ & & $\begin{array}{l}39.2^{\mathrm{c}}(\mathbf{6 e}) \\
42.4^{\mathrm{c}}(\mathbf{6 e})\end{array}$ & \\
\hline \multirow[t]{3}{*}{$\mathrm{Ir}_{4}\left(\mathrm{PH}_{3}\right)_{3}(\mathrm{CO})_{9}$} & $59.4(\mathbf{8 a})$ & $50.5(\mathbf{8 a})$ & $37.7(\mathbf{8 a})$ & $45.2(\mathbf{8 a})$ & $15.2(\mathbf{8 c})$ & $43.6(\mathbf{8 d})$ & \\
\hline & & $50.9(8 b)$ & $40.7(\mathbf{8 b})$ & $43.7(\mathbf{8 b})$ & & $38.7(\mathbf{8 e})$ & $17.4(8 \mathbf{e})$ \\
\hline & $36.5^{\mathrm{b}}(\mathbf{8 c})$ & & $45.9(8 c)$ & & & & \\
\hline $\mathrm{Ir}_{4}\left(\mathrm{PH}_{3}\right)_{4}(\mathrm{CO})_{8}$ & 60.3(10a) & & $47.0(\mathbf{1 0 a})$ & & $33.6(\mathbf{1 0 a})$ & & \\
\hline \multirow[t]{2}{*}{$\mathrm{Ir}_{4}\left(\mathrm{PH}_{3}\right)_{4}(\mathrm{CO})_{8}$} & $58.6(10 b)$ & & 46.4(10b) & 43.3(10b) & & & $27.4(\mathbf{1 0 d})$ \\
\hline & $36.9^{\mathrm{b}}(\mathbf{1 0 c})$ & & $41.0(\mathbf{1 0 c})$ & & $16.4(\mathbf{1 0 c})$ & & \\
\hline
\end{tabular}

${ }^{\mathrm{a}}$ Letters in parentheses correspond to structures in Figure $4 .{ }^{\mathrm{b}}$ A bridging $\mathrm{PH}_{3}$ changes into a equatorial one after the ligand dissociation. ${ }^{c}$ Isomers turn into $C_{3 v}$ structure after ligand dissociation 